\title{
THE GEODESIC HYPOTHESIS AND NON-TOPOLOGICAL SOLITONS ON PSEUDO-RIEMANNIAN MANIFOLDS
}

\author{
BY DAVID M.A. STUART
}

ABSTRACT. - A class of solitary wave solutions to a semi-linear wave equation on a pseudo-Riemannian manifold is studied. A construction of solutions which concentrate on geodesics is given.

(C) 2004 Elsevier SAS

RÉSUMÉ. - On étudie des ondes solitaires pour l'équation des ondes non linéaires sur une variété pseudoriemannienne. On construit des solutions avec concentration sur une courbe géodésique.

(C) 2004 Elsevier SAS

\section{Statement of results}

\subsection{Introduction}

The paper discusses a class of solutions, non-topological solitons, to the semi-linear wave equation $\square_{g} \phi+m^{2} \phi=\mathcal{F}(\phi)$ where $\phi$ is a complex function on $\mathbb{M}=\mathbb{R}^{1+n}$ and $\square_{g}$ is the wave operator on $\mathbb{R}^{1+n}$ defined with respect to the pseudo-Riemannian metric $g$. In the flat case, when $g$ is the Minkowski metric, the non-topological solitons are exact solutions of the form $e^{i \omega t} f_{\omega}(|x|)$ with $f_{\omega}$ positive; they are stable for certain $\omega$ depending upon the nonlinearity. The action of the Lorentz group gives solutions in which these solitons move along straight lines. In the presence of a non-flat metric it is proved that in the stable case under the rescaling $\underline{g} \rightarrow \frac{1}{\varepsilon^{2}} \underline{g}$, for $\varepsilon$ small, there exist solutions in which the solitons move along time-like geodesics, up to an error which is controlled in the energy norm for finite time. This result provides a mathematical justification of the geodesic hypothesis in general relativity for non-topological solitons with a given background metric. In a forthcoming article the analysis is extended to include the case in which the metric evolves according to the Einstein equation.

Non-topological solitons. To begin, we introduce a class of exact solutions to the semi-linear wave equation, on flat Minkowski space $\mathbb{M}=\mathbb{R}^{1+n}$, for a complex function $\phi: \mathbb{R}^{1+n} \rightarrow \mathbb{C}$,

$$
\partial_{t}^{2} \phi-\Delta \phi+m^{2} \phi=\mathcal{F}(\phi) .
$$

In the case when $\mathcal{F}$ factors as

$$
\mathcal{F}(\phi)=\beta(|\phi|) \phi, \quad \beta: \mathbb{R} \rightarrow \mathbb{R}, \quad \beta(0)=0
$$


there is a special class of solitary wave type solutions, called non-topological solitons, of the form

$$
\phi(t, x)=e^{i \omega t} f_{\omega}(x)
$$

where (for appropriate $\omega, \beta) f_{\omega}$ is the unique positive radial solution of

$$
-\Delta f_{\omega}+\left(m^{2}-\omega^{2}\right) f_{\omega}=\beta\left(f_{\omega}\right) f_{\omega} .
$$

Existence results for non-topological solitons (1.2) were developed in [18,2,3]. Uniqueness of the profile function $f_{\omega}$ has been proved for certain nonlinearities in [13], based on ideas in [5, 15] and others referenced therein. For $\omega^{2}<m^{2}$ the function $f_{\omega}$ is exponentially decaying and so the solutions (1.2) are spatially localised. This makes them an appropriate object of study for a mathematical analysis of the geodesic hypothesis, i.e. the problem of motion for particles in general relativity, as described in the next paragraph. In the Hamiltonian context solutions of the type of (1.2), in which the time evolution is along an orbit of the action of the symmetry group, are known as relative equilibria.

The geodesic hypothesis. Action of the Lorentz group on (1.2) gives a family of solutions to $(\mathrm{W})$ representing solitons moving along straight lines $x=u t$; see Section 1.5 for precise formulae. The main motivation for this paper is to understand what happens to these solutions when a pseudo-Riemannian metric $\underline{g}$ is introduced onto $\mathbb{M}$. In this setting the equation (W) generalises to

$$
\square_{\underline{g}} \phi+m^{2} \phi=\mathcal{F}(\phi), \quad \mathcal{F}(\phi)=\beta(|\phi|) \phi .
$$

(See (1.11) for the explicit form of $\square_{\underline{g}}$.) Under the rescaling $\underline{g} \rightarrow \varepsilon^{-2} \underline{g}$ the size of the soliton scales to $\mathrm{O}(\varepsilon)$, and it is in this limit that a reduced description is possible. The equation becomes:

$$
\square_{\underline{g}} \phi+\frac{m^{2}}{\varepsilon^{2}} \phi=\frac{1}{\varepsilon^{2}} \beta(|\phi|) \phi, \quad \mathcal{F}(\phi)=\beta(|\phi|) \phi .
$$

Corresponding to this scaling of the metric it is natural to introduce scaled Sobolev and Lebesgue norms $H_{\varepsilon}^{1}$ and $L_{\varepsilon}^{2}$ by

$$
\begin{gathered}
\|u\|_{L_{\varepsilon}^{2}}^{2}=\varepsilon^{-n} \int|u(x)|^{2} d x, \\
\|u\|_{H_{\varepsilon}^{1}}^{2}=\int_{\Sigma}\left(\varepsilon^{2-n}\left|\nabla_{x} u(x)\right|^{2}+\varepsilon^{-n}|u(x)|^{2}\right) d x .
\end{gathered}
$$

The main aim of this paper is to prove the following (stated here somewhat heuristically):

Given a time-like geodesic $\Gamma$ there exist $t_{*}>0$ and initial values $\left(\phi(0), \partial_{t} \phi(0)\right) \in H_{\varepsilon}^{1} \times L_{\varepsilon}^{2}$ such that for $\varepsilon$ sufficiently small $\left(\phi, \partial_{t} \phi\right)$ the solution to the corresponding Cauchy problem for $\left(W^{\varepsilon^{-2}} \underline{g}\right)$ is close in norm $C\left(\left[0, t_{*}\right] ; H_{\varepsilon}^{1} \times L_{\varepsilon}^{2}\right)$ to a non-topological soliton centred on $\Gamma$. The time $t_{*}>0$ is independent of $\varepsilon$ as $\varepsilon \rightarrow 0$.

Precise statements of the results are given in Sections 1.3, 1.6 and 1.7. In physical terms the meaning of the phrase "non-topological soliton centred on $\Gamma$ " is approximately as follows: in a local co-ordinate system corresponding to the reference frame of an observer moving along $\Gamma$, with the proper time taken as time-like co-ordinate, the solution looks like (1.2) to highest order 
(see Appendix A.11). A more precise formulation in mathematical terms is given in Section 1.3 using the co-ordinate system adapted to a foliation by space-like hypersurfaces. The difficulty of the problem arises from the need to obtain estimates on a fixed time interval $\left[0, t_{*}\right]$, uniform in $\varepsilon$, for solutions of the singular equation $\left(W^{\varepsilon^{-2}} \underline{g}\right)$ with initial data having singular behaviour as $\varepsilon \rightarrow 0$. Under rescaling this becomes a question of obtaining large (finite) time stability estimates for solutions in the presence of a slowly varying metric.

In general relativity the gravitational field is described by a pseudo-Riemannian metric on $\mathbb{M}$. A study of soliton solutions in $\left(W^{\varepsilon^{-2}} \underline{g}\right)$ thus corresponds to an investigation of the effect of a fixed gravitational field on the soliton. The soliton is strongly localised in space, and may be thought of as "particle-like". The expectation that the soliton moves along a time-like geodesic, in the limit in which its size is small compared to scales over which the metric varies, is natural in view of the principle of equivalence [26,14], and is sometimes known as the geodesic hypothesis. Thus the results of this paper give a rigorous validation of this hypothesis for the case of a given background metric (depending upon space and time).

So far $g$ has been a fixed background metric, corresponding to the notion of an external, or applied, gravitational field. In the general theory of relativity the metric $\underline{g}$ becomes a dynamical variable itself and evolves according to the Einstein equation:

$$
R_{\mu \nu}(\underline{g})-\frac{1}{2} R(\underline{g}) \underline{g}_{\mu \nu}=T_{\mu \nu}(\phi, \underline{g}) .
$$

Here $R_{\mu \nu}(g)$ is the Ricci curvature of the metric $g$ and its trace $R(g)$ is the scalar curvature. In the right side $T_{\mu \nu}$ is the stress-energy tensor; its precise form need not be given here. The Einstein equation is to be solved in conjunction with ( $\left.\mathrm{W}^{\underline{g}}\right)$ : together they form a quasilinear system which is essentially hyperbolic (modulo the usual proviso regarding gauge invariance.) In this situation it is to be expected that the geodesic hypothesis is still valid as long as the amplitude of the soliton is sufficiently small that its effect on the metric $g$ can be treated perturbatively. (This assumption is in addition to the one already introduced that the size of the soliton is small compared to other length scales in the problem.) Analogous results to those proved here for the Einstein-semi-linear wave system comprising $\left(\mathrm{W}^{\underline{g}}\right)$ and (1.6) will be presented in [21]; see the announcement [24]. A more extended introduction to the problem is given in the announcement [22].

Stability. Relation to other work. Stability of solitary waves in Hamiltonian systems such as (1.2) has been discussed in $[16,17,7,8]$. In particular explicit conditions on $\omega$ for stability in the radially symmetric case were given in $[16,17]$ for the case $\beta(|\phi|)=|\phi|^{p-1}$. The articles $[7$, 8] present a very general framework for stability analysis of this type of solution in infinite dimensional Hamiltonian systems. The present article extends techniques introduced in [23] to treat stability for (1.2) in a modulational fashion, i.e. to compute explicitly how the various parameters of the soliton evolve. This method gives somewhat stronger stability results for the flat space case than those obtained previously, and also generalises to allow a study of soliton-like solutions in the pseudo-Riemannian case, as carried out in this paper. The principal new feature in [23] is the use of symplectic structure to understand stability criteria at a linear level and to study modulation theory on a symplectic submanifold rather than just on the group orbit (see Section 1.5 for a discussion). A similar analysis for the corresponding solutions to the non-linear Schrödinger equation was given in [28] at the linearised level. The nonlinear wave equation case has additional complications arising in particular from the fact that the solutions (1.2) are only stable for $\omega$ lying in a certain interval $\mathbf{I}$ (see (1.35)). In the linear analysis this stability criterion arises from an interaction of the symplectic structure with the spectral analysis as described in Section 1.5. 
Apart from [20] the most closely related work to this appears to be [4,9] in which the motion of solitons in the non-linear Schrödinger equation

$$
i \varepsilon \partial_{t} \phi+\frac{\varepsilon^{2}}{2} \Delta \phi-V(x) \phi+|\phi|^{2} \sigma \phi=0
$$

is studied in the semi-classical limit $\varepsilon \rightarrow 0$. It is shown that the classical limit, i.e. the Hamiltonian flow determined by the Hamiltonian $H=P^{2} / 2+V(x)$, governs the soliton motion in this limit. Much shorter proofs are possible in the nonlinear Schrödinger case: one reason for this appears to be that the Galilean symmetry allows the estimates to be carried out in a frame in which the soliton is at rest. This does not seem to work in the present relativistically invariant setting, as the Lorentz transformations do not preserve the $t=$ constant hyperplanes or the Hamiltonian formulation. Another complicating factor here is of course the presence of variable coefficients in the principal term.

Organisation of the paper. The remainder of the article is arranged as follows: in Section 1.2 conditions required on the background metric are introduced so that an initial statement of the theorem can be given (Theorem 1.1). Further discussion of the stability properties of non-topological solitons is given in Sections 1.4-1.7 before giving a more general statement valid for a larger class of nonlinearities in the main theorem (Theorem 1.7) which is proved in Section 2. In fact the main theorem refers to a rescaled problem in which the soliton is "blown-up" to have size of $\mathrm{O}(1)$; the statement needs to be rescaled to give a result which implies Theorem 1.1, and this is finally done in Section 3. Some notation is summarised in Section 1.8 while various technical facts, formulae and calculations are given in the appendices.

\subsection{Assumptions on the metric}

Assume that the space-time $(\mathbb{M}, \underline{g})$ is diffeomorphic to $\left[0, t_{0}\right] \times \mathbb{R}^{n}$ and admits a foliation into space-like hypersurfaces which are the level sets of a time function $t$ :

$$
\mathbb{M} \approx\left[0, t_{0}\right] \times \Sigma, \quad \Sigma \approx \mathbb{R}^{n}, \quad \Sigma_{t_{1}} \equiv\left\{t_{1}\right\} \times \Sigma=t^{-1}\left(t_{1}\right)
$$

where $\approx$ means "is diffeomorphic to". It is assumed that there exists a co-ordinate system, $\left(x^{0}=t, x^{1}, \ldots, x^{n}\right)$, which will be fixed throughout this article, in which the metric $\underline{g}$ on $\mathbb{M}$ is of the form

$$
\underline{g}_{\mu \nu} d x^{\mu} d x^{\nu}=-p^{2} d t^{2}+g_{i j} d x^{i} d x^{j} .
$$

Here $p: \mathbb{M} \rightarrow \mathbb{R}$ is called the lapse function, while $g_{i j}(t, x)$ is the metric induced on $\Sigma_{t}$ from $\underline{g}$. Latin indices run from $1, \ldots, n$ and label the space-like co-ordinate functions $x^{1}, \ldots, x^{n}$ while Greek indices run from 0 to $n$ and label space-time co-ordinates. As usual the induced inner product on the cotangent space of $\Sigma_{t}$ is represented by the inverse matrix $g^{i j}(t, x)$ and the boldface notation $\mathbf{g}=\operatorname{det} g_{i j}$ will be used throughout for the integration density.

It will be assumed that the co-tangent bundles of the slices $\Sigma_{t}$ admit orthonormal frames, i.e. $n$ mutually orthogonal one-forms $\left\{A^{I}(t, x)\right\}_{I=1}^{n}$ of unit length (a triad); upper case roman letters $I, J, \ldots$ will be used to label these. Thus for each $x \in \Sigma_{t}$ there exists an isometry

$$
\begin{aligned}
A(t, x): T_{x} \Sigma & \rightarrow \mathbb{R}^{n} \\
V^{i} \partial_{i} & \mapsto A_{i}^{I}(t, x) V^{i},
\end{aligned}
$$


where $\mathbb{R}^{n}$ is given the standard inner product

$$
X \cdot Y=\sum_{I=1}^{N} X^{I} Y^{I}=X^{I} Y_{I} .
$$

(Using this inner product there is no need to distinguish between upper and lower indices, so that when $I, J, \ldots$ appear they may do so as either an upper or a lower index.) It follows that $\sum_{I=1}^{n} A_{i}^{I} A_{j}^{I}=g_{i j}$ so that $A$ can be thought of as a square root of $g$.

The following hypotheses ${ }^{1}$ will be made: there exist positive numbers $K_{0}, K_{1}, K_{2}, \ldots$ such that

(M-1) $p^{2}, g$ are of class $C^{s}$ with all derivatives up to $s$ th order bounded by $K_{s}^{2}$.

(M-2) $A$ and $A^{-1}$ are of class $C^{s}$ with derivatives up to $s$ th order bounded by $K_{s}$.

(M-3) $p^{2 n}$ and $\mathbf{g}=\operatorname{det} g_{i j}$ are $\geqslant 1 / K_{0}^{2 n}>0$ everywhere. $^{2}$

In terms of the notation defined in Section 1.8 this hypothesis, for $s=2$, means that $g \in \mathcal{M} e t_{0}^{2, K_{2}}$ and $\underline{g} \in \mathcal{M} e t^{2, K_{2}}$.

Remark. - As regards specifying dependences on $g$ and $A$ of various functions which will be introduced the convention will be adopted of only specifying explicitly $g$, with dependence on $A$ then understood. In fact, since the co-ordinate system is fixed, $A$, thought of as a matrix, can be taken to be the unique positive symmetric square root of the positive symmetric matrix $g$.

Time-like geodesics on $(\mathbb{M}, \underline{g})$ can be identified, via the foliation (1.7), with curves $t \mapsto(t, \xi(t)) \in \mathbb{M}$ where the curve $t \mapsto \xi(t) \in \Sigma$ is a solution of the equation

$\left(\mathrm{GEO}^{\underline{g}}\right)$

$$
\frac{d}{d t}\left(\frac{\gamma u_{k}}{q}\right)+\gamma q_{, k}+\frac{\gamma u_{i} u_{j}}{2 q} h_{, k}^{i j}=0 .
$$

In this equation the velocity is $u^{j}=\frac{d \xi^{j}}{d t}$ and $q, h$ are metric coefficients evaluated along the curve:

$$
\begin{aligned}
q(t) & =p(t, \xi(t)), & q_{, k}(t) & =\frac{\partial p}{\partial x^{k}}(t, \xi(t)), \\
h_{i j}(t) & =g_{i j}(t, \xi(t)), \quad & h_{, k}^{i j}(t) & =\frac{\partial g^{i j}}{\partial x^{k}}(t, \xi(t)) .
\end{aligned}
$$

Since $u \in T_{\xi} \Sigma$ the index is lowered with $h$ :

$$
u_{j}(t)=h_{j k}(t) u^{k}(t)
$$

The notation $\gamma(t)$ is for the Lorentz contraction factor ${ }^{3}$

$$
\gamma(t)=\bar{\gamma}\left(|u(t)|_{h} / q\right)
$$

where $\bar{\gamma}(s) \equiv\left(1-s^{2}\right)^{-1 / 2}$.

The wave equation $\left(\mathrm{W}^{\underline{g}}\right)$ on $(\mathbb{M}, \underline{g})$ is:

$$
\partial_{t}\left(\frac{\sqrt{\mathbf{g}}}{p} \partial_{t} \phi\right)-\partial_{i}\left(p \sqrt{\mathbf{g}} g^{i j} \partial_{j} \phi\right)+p \sqrt{\mathbf{g}}\left(m^{2} \phi-\beta(|\phi|)\right) \phi=0 .
$$

\footnotetext{
${ }^{1}$ Although in this article $s=2$ it is convenient to introduce explicitly constants $K_{s}$ which control the $s$ th order derivatives in order to keep track of where differing levels of smoothness are required in different parts of the analysis.

${ }^{2}$ The powers of $K_{s}$ are just to ensure scale invariance of (M-1)-(M-3).

${ }^{3}$ The time dependence of $q$ and $h$ is suppressed here. 


\subsection{Theorem regarding geodesic motion on $\left(\mathbb{M}, \varepsilon^{-2} \underline{g}\right)$ (initial statement)}

In this section a theorem will be stated which illustrates the type of result which will be proved later on in more generality in this paper. Consider, for simplicity, the case of pure power nonlinearity in three spatial dimensions $(n=3)$. Rescale the metric $\underline{g} \rightarrow \frac{1}{\varepsilon^{2}} \underline{g}$ for $\varepsilon$ small: in this limit the solution (1.2) is exponentially localised in a region of size $\bar{\varepsilon}$, which is small compared to length scales over which the metric varies. Under this scaling equation (1.11) becomes:

$$
\partial_{t}\left(\frac{\sqrt{\mathbf{g}}}{p} \partial_{t} \phi\right)-\partial_{i}\left(p \sqrt{\mathbf{g}} g^{i j} \partial_{j} \phi\right)+\frac{1}{\varepsilon^{2}} p \sqrt{\mathbf{g}}\left(m^{2} \phi-\beta(|\phi|)\right) \phi=0 .
$$

In the following formula $u(t), \gamma(t), q(t), h(t)$ are as in (1.10) and $P_{t}=P_{u(t), h(t)}, Q_{t}=Q_{u(t), h(t)}$ where $P_{u, h}$ is the projection operator in the direction $u$ relative to the inner product $h$, and $Q_{u, h}$ is its complement (see Appendix A.2 for explicit formulae). The following formula gives a function which represents a soliton (1.2) centred on the curve $t \mapsto \xi(t)$ :

$$
\begin{aligned}
\phi_{0}^{\varepsilon}(t, x) \equiv & f_{\omega}\left(\frac{1}{\varepsilon}\left|\gamma P_{t}(x-\xi)+Q_{t}(x-\xi)\right|_{h}\right) \\
& \times \exp \left[\frac{i}{\varepsilon}\left(\int_{0}^{t} \frac{\omega q}{\gamma} d t^{\prime}+\eta-\frac{\gamma}{q}\langle x-\xi, u\rangle_{h}\right)\right]
\end{aligned}
$$

where $\gamma=\bar{\gamma}\left(|u|_{h} / q\right)$, the $t$ dependence of $u, h, q, \xi, \gamma$ being suppressed. For a given $C^{1}$ function $t \mapsto(\omega, \eta, \xi, u)$ the function $\phi_{0}^{\varepsilon}$ is also $C^{1}$ and at fixed time $t$ lies in $H^{1}\left(\mathbb{R}^{n}\right)$.

Remark. - The formula for $\phi_{0}^{\varepsilon}$ is a quite natural generalisation of the exact solution obtained by Lorentz transformation of the solution $e^{i \omega t} f_{\omega}(x)$ in the flat case (see Sections 1.5 and 2.1). There exists a local co-ordinate system $(\hat{t}, \hat{x})$ in a tube around the curve in which $\phi_{0}(\hat{t}, \hat{x}) \sim e^{i \omega \hat{t}} f_{\omega}(\hat{x})$ (see Appendix A.11). This co-ordinate system is the proper reference frame of an accelerated observer moving with the soliton [14, Section 13.6]. The formula is not co-ordinate invariant due to the presence of, for example, the expression $x-\xi$. The exponential localisation of $f$ and its derivatives means that up to a controlled error it would be possible to convert $\phi_{0}^{\varepsilon}$ into a more invariant form by systematic use of the exponential mapping. However, this would complicate notation without changing the essential content of the results, so it seems better to fix a system of co-ordinates throughout in terms of which the metric is as in (1.8) and proceed as in (1.12).

It should be emphasized that so far (1.12) is just a definition: it is not known to be a solution to $\left(1.11^{\varepsilon}\right)$ except in the flat case. The next theorem says essentially that under certain stability hypotheses if $t \mapsto \xi(t)$ is a geodesic and $\varepsilon<<1$ there does exist a solution to $\left(1.11^{\varepsilon}\right)$ close to (1.12) in energy norm for a finite time uniform in $\varepsilon$.

THEOREM 1.1 (Geodesic motion on $\left(\mathbb{M}, \varepsilon^{-2} \underline{g}\right)$ ). - Assume $n=3$ and that $\underline{g}$ is a metric on $\mathbb{M}$ satisfying the conditions (M-1)-(M-3) in Section 1.2 for $s=0,1,2$ and let $H_{\varepsilon}^{1}, L_{\varepsilon}^{2}$ be the scaled spaces defined in (1.4)-(1.5). Let $t \mapsto \xi^{(0)}(t) \in \Sigma$ be a time-like geodesic as described in Section 1.2 and assume that $\beta(|\phi|)=|\phi|^{p-1}$ with $2 \leqslant p<1+4 / n=7 / 3$. For $\omega(0) \in \mathbf{I}$, where $\mathrm{I}$ is the non-empty interval defined in (1.35), there exist

(i) positive numbers $c_{*}>0, t_{*} \in\left(0, t_{0}\right], \varepsilon_{*}>0$,

(ii) a $C^{1}$ function $(\omega, \eta, \xi, u) \in C^{1}\left(\left[0, t_{*}\right] ; \mathbb{R}^{2} \times T \Sigma\right)$,

(iii) and initial data $\left(\phi^{\varepsilon}(0), \phi_{t}^{\varepsilon}(0)\right) \in H_{\varepsilon}^{1} \times L_{\varepsilon}^{2}$, 
such that for all $0 \leqslant t \leqslant t_{*}$ and $0<\varepsilon<\varepsilon_{*}$,

$$
\varepsilon^{-2}|\omega(t)-\omega(0)|+\varepsilon^{-2}|\eta(t)-\eta(0)|+\varepsilon^{-1}\left|\xi(t)-\xi^{(0)}(t)\right|+\varepsilon^{-1}\left|u(t)-\frac{d \xi^{(0)}}{d t}\right| \leqslant c_{*}
$$

The corresponding solution to $\left(1.11^{\varepsilon}\right)$ satisfies, for all $0 \leqslant t \leqslant t_{*}$ and $0<\varepsilon<\varepsilon_{*}$, the estimate

$$
\left\|\phi^{\varepsilon}(t, x)-\phi_{0}^{\varepsilon}(t, x)\right\|_{H_{\varepsilon}^{1}}+\varepsilon\left\|\partial_{t}\left(\phi^{\varepsilon}(t, x)-\phi_{0}^{\varepsilon}(t, x)\right)\right\|_{L_{\varepsilon}^{2}} \leqslant c_{*} \varepsilon
$$

with $\phi_{0}^{\varepsilon}$ as in (1.12). The theorem is also valid in arbitrary space dimension for the large class of nonlinearities $\beta$ described in Section 1.7.

Proof. - This is proved in Section 3 in a somewhat generalised form.

Remarks. - (i) The norm in (1.14) is stronger than the energy norm $H^{1} \times L^{2}$ since $n=3$. Observe that the time interval is fixed for $0<\varepsilon<\varepsilon_{*}$ while the solutions are singular, hence the difficulty of the problem. Alternatively under the rescaling in Section 1.6 the result is turned into a type of (generalised) stability theorem (with modulation) for solitons on large time intervals $\left[0, t_{*} / \varepsilon\right]$, acted on by a slowly varying metric $g(\varepsilon t, \varepsilon x)$. Indeed it will be more convenient to carry out the proofs for the rescaled problem introduced in Section 1.6; this implies Theorem 1.1 in a straightforward way (see Section 3).

(ii) The condition that $\omega(0) \in \mathbf{I}$ is crucial, and ensures that the solution (1.2) at frequency $\omega(0)$ is stable with respect to the equation (W) on flat Minkowski space. The upper bound on $p$ ensures the interval $\mathbf{I}$ is non-empty, while the lower bound on $p$ is imposed for regularity reasons. The proof depends on a modulational approach to understanding the stability condition which was developed in [23] and is summarised in Section 1.5.

(iii) Also it is worth emphasizing that the behaviour described in the theorem is stable with respect to perturbation of the initial data in the sense that the theorem is valid for initial data lying in a sufficiently small ball (with respect to the norm $H_{\varepsilon}^{1} \times L_{\varepsilon}^{2}$ ): see Theorem 1.7.

\subsection{First order formulation}

The wave equation. In first order formulation (W $\underline{g})$ takes the form

$\left(\mathrm{NLW}^{\underline{g}}\right)$

$$
\begin{aligned}
\frac{\partial \phi}{\partial t} & =\frac{p}{\sqrt{\mathbf{g}}} \psi, \\
\frac{\partial \psi}{\partial t} & =\Delta_{p, g} \phi-p \sqrt{\mathbf{g}}\left(m^{2} \phi-\beta(|\phi|) \phi\right),
\end{aligned}
$$

where

$$
\Delta_{p, g} \phi=\frac{\partial}{\partial x^{i}}\left(p \sqrt{\mathbf{g}} g^{i j} \frac{\partial \phi}{\partial x^{j}}\right)
$$

Geodesics in terms of the cotangent space variables. It is also useful to write the geodesic equation in first order form: introduce a conjugate momentum variable $\pi \in T_{\xi}^{*} \Sigma$, where $T_{\xi}^{*} \Sigma$ is the cotangent space to $\Sigma$ at $\xi$, defined by: ${ }^{4}$

$$
\pi_{j}=\pi_{j}(u, \underline{h}) \equiv \frac{\bar{\gamma}\left(|u|_{h} / q\right)}{q} u_{j} \quad\left(\text { where } u_{j}=h_{j k} u^{k} \text { and } \underline{h}=-q^{2} d t^{2}+h_{i j} d x^{i} d x^{j}\right) .
$$

\footnotetext{
${ }^{4}$ The function $\bar{\gamma}$ was defined just before (1.11).
} 
In terms of $\pi$ the geodesic equation (GEO $\underline{g})$ can be written as a first order system:

$$
\begin{aligned}
\frac{d \xi^{j}}{d t} & =\frac{q h^{j k}}{\sqrt{1+|\pi|_{h}^{2}}} \pi_{k}, \\
\frac{d \pi_{k}}{d t} & =-\sqrt{1+|\pi|_{h}^{2}} q_{, k}-\frac{q h_{, k}^{j l}}{2 \sqrt{1+|\pi|_{h}^{2}}} \pi_{j} \pi_{l} .
\end{aligned}
$$

Under the assumptions (M-1)-(M-3) for $s=0,1,2$ the right side of (1.17) is Lipschitz for $\pi$ bounded which makes these variables more convenient for comparison purposes (see Section 2.6).

\subsection{Exact solutions on flat space and review of stability theory}

The main objective of this section is to review the approach to stability theory given in [23]. First it is necessary to give explicit formulae for the full set of solutions in flat space which are obtained by applying the Poincare group to (1.2); the final answer is (1.25). Although the formulae are complicated they are simply obtained by first changing variables so the metric is of the form (1.18) and then applying Lorentz transformations.

Existence. Consider the case of a flat space-time $\mathbb{M}$ in which the functions $p, g$ defining the metric $\underline{g}$ take constant values $q, h$, thus defining a flat metric

$$
\underline{h}=-q^{2} d t^{2}+h_{i j} d x^{i} d x^{j}
$$

with, in the notation defined in Section $1.8, \underline{h} \in \operatorname{Met}^{K}$ for some $K>0$. Time-like curves in $\mathbb{M}$ may be parametrised by $t$ and written $t \mapsto(t, \xi(t))$ with $\xi \in \Sigma=\mathbb{R}^{n}$; the space-time velocity is then the four-vector $\left(1, u=\frac{d \xi}{d t}\right)$ with $u \in T_{\xi} \Sigma$ satisfying $|u|_{h}<q$. In the flat case the action of the Poincaré group on (1.2) gives rise to a $(2 n+2)$ dimensional family of solutions centred along straight lines: the first two parameters are the frequency $\omega$ and a phase factor $\theta$ while the remaining $2 n$ parameters are the centre $\xi \in \Sigma$ and the velocity $u=\frac{d \xi}{d t} \in T_{\xi} \Sigma$. Thus define the parameter space

$$
O^{\underline{h}} \equiv\left\{\lambda=(\omega, \theta, \xi, u) \in \mathbb{R}^{2} \times T \Sigma:|\omega|<m \text { and }|u|_{h}<q\right\} .
$$

Let $\lambda=(\omega, \theta, \xi, u) \in C^{1}\left(\mathbb{R} ; O^{H}\right)$ and introduce as in (1.9) an isomorphisn

$$
B_{i}^{I}: V^{i} \mapsto B_{i}^{I} V^{i}
$$

between $\left(T_{x} \Sigma, h\right)$ and $\mathbb{R}^{n}$ with the standard inner product, so that

$$
h^{i j} B_{i}^{I} B_{j}^{J}=\delta^{I J} .
$$

(In this case $B_{i}^{I}$ is independent of $(t, x)$ of course.) The convention introduced in the remark following (M-3) will be extended here so that for quantities depending on $h, B$ only the dependence on $h$ will be explicitly designated.

Now define

(1.21) $\gamma=\bar{\gamma}\left(|u|_{h} / q\right)$,

(1.22) $\quad Z^{I}(t, x)=\mathbf{Z}^{I}(x ; \lambda(t), \underline{h}) \quad$ where $\mathbf{Z}^{I}(x ; \lambda, \underline{h}) \equiv B_{i}^{I}\left(\gamma P_{u, h}(x-\xi)+Q_{u, h}(x-\xi)\right)^{i}$, 


$$
\Theta(t, x)=\boldsymbol{\Theta}(x ; \lambda(t), \underline{h}) \quad \text { where } \boldsymbol{\Theta}(x ; \lambda, \underline{h}) \equiv \theta-\frac{\omega}{q} \mathbf{Z}(x ; \lambda, \underline{h}) \cdot(B u) .
$$

Remark. - The function $(x, \lambda, \underline{h}) \mapsto \mathbf{Z}$ (resp. $\mathbf{\Theta})$ is smooth to $\mathbb{R}^{n}$ (resp. $\mathbb{R}$ ) for $\underline{h}$ in any Met $^{K}$ (see Section 1.8) and $\lambda \in O^{\underline{h}}, x \in \mathbb{R}^{n}$. The way to understand these formulae is to consider the case $\xi=u t$ and $\theta=\omega q t / \gamma$ : in this case they are essentially the Lorentz transformations of $x$ and $\omega t$ (apart from a linear transformation due to the non-standard form of the metric (1.18)). With this said the next lemma, which follows from a straightforward calculation using formulae in Appendix A.3, is not unexpected.

\section{LEMMA 1.2. - Assume there is given}

(i) a constant coefficient metric $\underline{h}=-q^{2} d t^{2}+h_{i j} d x^{i} d x^{j}$,

(ii) a function $f_{\omega}$ which verifies (1.3),

(iii) a function $\lambda=(\omega, \theta, \xi, u) \in C^{1}\left(\mathbb{R} ; O^{\underline{h}}\right)$ which satisfies

$$
\frac{d \lambda}{d t}=\mathbf{V}_{\mathbf{0}}(\lambda ; \underline{h})=\left(0, \frac{\omega q}{\gamma}, u, 0\right)
$$

Then the pair of functions

$$
\begin{aligned}
\phi(t, x) & =\phi_{S}(x ; \lambda(t), \underline{h}) \quad \text { where } \phi_{S}(x ; \lambda, \underline{h}) \equiv f_{\omega}(\mathbf{Z}) e^{i \Theta}, \\
\psi(t, x) & =\psi_{S}(x ; \lambda(t), \underline{h}) \quad \text { where } \\
\psi_{S}(x ; \lambda, \underline{h}) & \equiv\left(i \omega \gamma \sqrt{\mathbf{h}} f_{\omega}(\mathbf{Z})-\frac{\gamma \sqrt{\mathbf{h}}}{q}(B u) \cdot \nabla_{Z} f_{\omega}(\mathbf{Z})\right) e^{i \Theta}
\end{aligned}
$$

with $\gamma, \mathbf{Z}, \Theta$ as in (1.21)-(1.23), is a solution of (NLW $\underline{h})$.

The space of soliton solutions is defined to be:

$$
\mathcal{S} \equiv\left(\phi_{S}(\cdot ; \lambda, \underline{h}), \psi_{S}(\cdot ; \lambda, \underline{h})\right) \subset H^{1} \times L^{2}
$$

for $\lambda \in O^{\underline{h}}$. Hypotheses on the smoothness of the map $(\lambda, \underline{h}) \mapsto\left(\phi_{S}(\cdot ; \lambda, \underline{h}), \psi_{S}(\cdot ; \lambda, \underline{h})\right)$ appropriate for this article are given in $(\mathrm{R})$ in Section 1.7.

It is also useful to rewrite (1.24) in terms of the momentum variable $\pi$ which takes values in the cotangent space (see Section 1.4). So for $(\xi, u) \in T \Sigma$ and at time $t$ let the momentum variable be given by

$$
\pi=\pi(u, \underline{h}(t)) \in T_{\xi}^{*} \Sigma \quad(\text { with } \underline{h}=\underline{g}(t, \xi(t)))
$$

where the function $\pi(u, \underline{h})$ was defined in (1.16). Now introduce ${ }^{5}$

$$
\lambda^{*}=\mathcal{L}(\lambda, \underline{h})=\mathcal{L}_{\underline{h}}(\lambda)=(\omega, \theta, \xi, \boldsymbol{\pi}(u, \underline{h})) \in \mathbb{R}^{2} \times T^{*} \Sigma .
$$

(1.24) is equivalent to the following equation for $\lambda^{*}$ :

$$
\frac{d \lambda^{*}}{d t}=\mathbf{V}_{0}^{*}\left(\lambda^{*} ; \underline{h}\right) \equiv\left(0, \frac{\omega q}{\sqrt{1+|\pi|_{h}^{2}}}, \frac{h^{j k} \pi_{k} \partial_{j}}{\sqrt{1+|\pi|_{h}^{2}}}, 0\right)
$$

\footnotetext{
${ }^{5}$ The transformation $\mathcal{L}_{\underline{h}}: \mathbb{R}^{2} \times T \Sigma \rightarrow \mathbb{R}^{2} \times T^{*} \Sigma$ taking $\lambda \rightarrow \mathcal{L}_{\underline{h}}(\lambda)$ is clearly closely related to the Legendre transformation $T \Sigma \rightarrow T^{*} \bar{\Sigma}$. 
(where $\partial_{j}$ is the standard co-ordinate basis for $T_{\xi} \Sigma$.) Thus $\lambda^{*}$ provides an alternative system of co-ordinates for describing the solitons. The inverse of the map $\lambda \mapsto \lambda^{*}=\mathcal{L}_{\underline{h}}(\lambda)$ will be written

$$
\lambda=\mathcal{L}^{-1}\left(\lambda^{*}, \underline{h}\right)=\mathcal{L}_{\underline{h}}^{-1}\left(\lambda^{*}\right) .
$$

Lagrange multipliers and the augmented Hamiltonian. Let $\underline{h}$ be a constant coefficient metric as in (1.18). It is useful to note, at the formal level, that $\left(\phi_{S}, \overline{\psi_{S}}\right)$ is a critical point of

$$
H(\phi, \psi)=\frac{1}{2} \int_{\mathbb{R}^{n}}\left[\frac{1}{\sqrt{\mathbf{h}}}|\psi|^{2}+\left(|\nabla \phi|_{h}^{2}+m^{2}|\phi|^{2}-G(|\phi|)\right) \sqrt{\mathbf{h}}\right] q d x,
$$

where $G(\tau)=\int_{0}^{\tau} 2 s \beta(s) d s$, subject to the constraints that $\Pi, Q$ be fixed, where

$$
\begin{aligned}
& \Pi_{i}(\phi, \psi)=\int\left\langle\psi, \frac{\partial \phi}{\partial x^{i}}\right\rangle d x \quad \text { (momentum), } \\
& Q(\phi, \psi)=\int\langle i \psi, \phi\rangle d x \quad \text { (charge). }
\end{aligned}
$$

These are the conserved quantities deriving from translation and phase $\left(S^{1}\right)$ invariance on account of Noether's theorem. In this setting $u^{i}$ and $\omega q / \gamma$ emerge as the corresponding Lagrange multipliers and thus the pair $\left(\phi_{S}, \psi_{S}\right)$ is a critical point of the enlarged functional

$$
F(\phi, \psi ; \lambda)=H(\phi, \psi)+u^{i} \Pi_{i}(\phi, \psi)+\frac{\omega q}{\gamma} Q(\phi, \psi) .
$$

This quantity is called the augmented Hamiltonian. The Hessian of $F$ at $\left(\phi_{S}(\cdot, \lambda), \psi_{S}(\cdot, \lambda)\right)$ is an important quantity in the stability analysis and much studied in the Hamiltonian literature (see e.g. [12]).

Stability. The stability of the solutions just described depends in a crucial way on $\omega$. Define the stability interval to be:

$$
\mathbf{I} \equiv\left\{\omega: \boldsymbol{\mu}_{\boldsymbol{\omega}} \equiv \frac{\partial}{\partial \omega}\left(-\omega\left\|f_{\omega}\right\|_{L^{2}}^{2}\right)>0\right\}
$$

It follows from a simple scaling argument (see [16]) that when $\beta(|\phi|)=|\phi|^{p-1}$, with $1<p<1+\frac{4}{n}$,

$$
\mathbf{I}=\left\{\omega: \frac{1}{1+\frac{4}{p-1}-n}<\frac{\omega^{2}}{m^{2}}<1\right\}
$$

while $\mathbf{I}$ is empty if $p$ lies outside this range. The condition in (1.35)-(1.36) first appeared in [16] and has been shown to be essentially equivalent to stability in the spherically symmetric case; see also $[7,8,19]$ for general stability theorems for solitary waves. In [23] a modulational approach to stability of (1.2) was developed which gives explicit information on the time evolution of the parameters, and is hence useful in situations which are dynamically non-trivial as is the case in this paper. In the context of a flat space-time the following stability theorem was proved:

THEOREM 1.3 [23]. - Consider the Cauchy problem for (NLW $\underline{h}$ ) with flat metric as in (1.18), $\beta(|\phi|)=|\phi|^{p-1}$, and initial values $(\phi(0, \cdot), \psi(0, \cdot)) \in H^{1} \times L^{2}$. Define

$$
O_{\text {stab }}^{\underline{h}} \equiv\left\{\lambda=(\omega, \theta, \xi, u) \in O^{\underline{h}} \text { and } \omega \in \mathbf{I}\right\} .
$$


Then for all $\lambda_{0} \in O_{\text {stab }}$ there exists $\varepsilon_{*}=\varepsilon_{*}\left(\lambda_{0}\right)>0$ such that if

$$
\varepsilon=\left\|\phi(0, \cdot)-\phi_{S}\left(\cdot ; \lambda_{0}, \underline{h}\right)\right\|_{H^{1}}+\left\|\psi(0, \cdot)-\psi_{S}\left(\cdot ; \lambda_{0}, \underline{h}\right)\right\|_{L^{2}}<\varepsilon_{*}\left(\lambda_{0}\right)
$$

then there exist $\lambda \in C^{1}(\mathbb{R} ; O$ stab $),(\phi, \psi) \in C\left(\mathbb{R} ; H^{1} \times L^{2}\right)$ and $c_{1}>0$ such that

$$
\sup _{t \in \mathbb{R}}\left(\left\|\phi(t, \cdot)-\phi_{S}(\cdot ; \lambda(t), \underline{h})\right\|_{H^{1}}+\left\|\psi(t, \cdot)-\psi_{S}(\cdot ; \lambda(t), \underline{h})\right\|_{L^{2}}\right)<c_{1} \varepsilon .
$$

The curve $t \mapsto \lambda(t)$ is the solution of an explicitly determined system of ordinary differential equations and there exists $c_{2}>0$ such that

$$
\left|\partial_{t} \lambda-\mathbf{V}_{\mathbf{0}}(\lambda ; \underline{h})\right|+|\omega(t)-\omega(0)|+|u(t)-u(0)| \leqslant c_{2} \varepsilon .
$$

Even in the flat case this theorem generalises previous results in that stability is proved without the assumption of radial symmetry and also for solitons moving at arbitrary velocities $u$. The main point, however, is to get explicit information on the curve $t \mapsto \lambda(t)$ since it is the corresponding set of ODE's derived in Section 2.2 of this paper which implies geodesic motion.

Since the techniques used in this paper are a generalisation of those in [23] to the pseudoRiemannian case it is worth saying a few words about the proof of Theorem 1.3. The main point is to use the symplectic structure to explain at a linear level the origin of the stability condition (1.35). This corresponds to a "symplectification" of the usual approach, in that the $2 n+2$ parameters $(\omega, \theta, \xi, u)$ which make up $\lambda$ are allowed to modulate rather than just the translation and phase variables $(\theta, \xi)$ as in the standard approach. The set of soliton solutions $\mathcal{S}$ turns out to be a local symplectic submanifold in the stable case, a fact which is crucial to the argument.

To see this clearly write the solution in the form

$$
\phi(t)=\phi_{S}(x ; \lambda(t), \underline{h}(t))+\tilde{\phi}(t), \quad \psi(t)=\psi_{S}(x ; \lambda(t), \underline{h}(t))+\tilde{\psi}(t) .
$$

The purpose is to determine $\lambda(t)$ so that the error terms $\tilde{\phi}, \tilde{\psi}$ can be estimated in $H^{1} \times L^{2}$ uniformly in time. This is achieved by using conservation laws for $H, Q, \Pi$ in conjunction with the following infinitesimal stability lemma. Let $\underline{h}$ be flat as in (1.18) and for fixed $\lambda=(\omega, \theta, \xi, u) \in O^{\underline{h}}$ consider the quadratic form

$$
\begin{aligned}
\mathcal{E}(\tilde{\phi}, \tilde{\psi} ; \lambda, \underline{h})= & \frac{1}{2} \int\left(\frac{q}{\sqrt{\mathbf{h}}}|\tilde{\psi}|^{2}+q \sqrt{\mathbf{h}}\left(\left|\left(\nabla_{x}\right)_{i} \tilde{\phi}\right|_{h}^{2}+m^{2}|\tilde{\phi}|^{2}-\beta\left(\left|\phi_{S}\right|\right)|\tilde{\phi}|^{2}\right)\right. \\
& \left.-q \sqrt{\mathbf{h}} \beta^{\prime}\left(\left|\phi_{S}\right|\right) \frac{\left\langle\phi_{S}, \tilde{\phi}\right\rangle^{2}}{\left|\phi_{S}\right|}-\frac{2 \omega q}{\gamma}\langle\tilde{\psi}, i \tilde{\phi}\rangle+2 u^{i}\left\langle\left(\nabla_{x}\right)_{i} \tilde{\phi}, \tilde{\psi}\right\rangle\right) d x
\end{aligned}
$$

with $\phi_{S}=\phi_{S}(x ; \lambda, \underline{h})$ defined as in (1.25). The significance of the quantity $\mathcal{E}$ is that it is the Hessian of the augmented Hamiltonian defined above. Introduce the subspace:

$$
\Upsilon_{\lambda, \underline{h}} \equiv\left\{(\tilde{\phi}, \tilde{\psi}) \in H^{1} \times L^{2}: \Omega\left((\tilde{\phi}, \tilde{\psi}), \frac{\partial}{\partial \lambda_{A}}\left(\phi_{S}, \psi_{S}\right)\right)=0 \text { for all } A\right\}
$$

where $\Omega$ is the standard symplectic form for wave equations i.e.,

$$
\begin{aligned}
& \boldsymbol{\Omega}:\left(L^{2}(\Sigma ; \mathbb{C})\right)^{2} \times\left(L^{2}(\Sigma ; \mathbb{C})\right)^{2} \rightarrow \mathbb{R}, \\
& \boldsymbol{\Omega}\left((\tilde{\phi}, \tilde{\psi}),\left(\phi^{\prime}, \psi^{\prime}\right)\right)=\int\left(\left\langle\tilde{\phi}, \psi^{\prime}\right\rangle-\left\langle\tilde{\psi}, \phi^{\prime}\right\rangle\right) d x .
\end{aligned}
$$


As remarked above the stability condition $\omega \in \mathbf{I}$ ensures that $\mathcal{S}$ is a local symplectic manifold in a neighbourhood of the corresponding point $\left(\phi_{S}(\lambda, \underline{h}), \psi_{S}(\lambda, \underline{h})\right)$ (where as usual $\lambda=(\omega, \theta, \xi, u))$. The geometrical interpretation of (1.42) is then that $\Upsilon_{\lambda, \underline{h}}$ is the symplectic normal space to $\mathcal{S}$ at the point $\left(\phi_{S}(\lambda, \underline{h}), \psi_{S}(\lambda, \underline{h})\right)$. The next lemma indicates the analytical significance of the symplectic normal subspace in providing a linear or infinitesimal interpretation of the stability conditions in (1.35). In the following recall that $\beta(|\phi|)=|\phi|^{p-1}$ and that $O_{\text {stab }}^{\underline{h}}$ consists of those $\lambda \in O^{\underline{h}}$ with $\omega \in \mathbf{I}$ :

LEMMA 1.4. - The quadratic form $\mathcal{E}(\tilde{\phi}, \tilde{\psi} ; \lambda, \underline{h})$, restricted to the subspace $\Upsilon_{\lambda, \underline{h}}$, defined in (1.42), is equivalent to the $H^{1} \times L^{2}$ norm, uniformly for $\lambda$ in compact subsets of the set $O_{\mathrm{stab}}$ defined in (1.37).

This lemma suggests a method of proof for Theorem 1.3: try to decompose the solution as in (1.40) with $(\tilde{\phi}, \tilde{\psi}) \in \Upsilon_{\lambda(t), \underline{h}}$, and use the foregoing lemma in conjunction with the conservation laws for $H, Q, \Pi$ to obtain $H^{1} \times L^{2}$ estimates. This turns out to be possible: one crucial point is that the stability condition ensures that $\mathcal{S}$ is symplectic which means that the hoped for decomposition is possible by a symplectic tubular neighbourhood theorem. The details are given in [23].

Proof of Lemma 1.4. - The result as stated can be reduced to the case $q=1, h_{I J}=\delta_{I J}$ by a linear change of variables: let $B$ be as in (1.20) and define $\tilde{x}^{I}=B_{j}^{I} x^{j}, U=(B u) / q$. In the case $q=1, h_{i j}=\delta_{i j}$ it is proved in [23, Theorems 1.3 and 2.7]. In fact in the case $\beta(|\phi|)=|\phi|^{p-1}$ two proofs are given, one using the fact that the $f_{\omega}$ are then optimizers of the Gagliardo-Nirenberg inequality ([27]).

Remark. - In fact Lemma 1.4 holds for a very large class of nonlinearities $\beta$ described in [23]. In this article its validity will be required (see hypothesis (IS) below) as a structural assumption on the nonlinearity.

\subsection{Statement of theorem in pure power case with $n=3$ (rescaled version)}

In this section a rescaled problem is introduced which is related to the original problem by a simple rescaling of $(t, x)$ (see Section 3). In this scaling the soliton has finite $(\mathrm{O}(1))$ size and the metric varies on length scales of $\mathrm{O}(1 / \varepsilon)$. It is this formulation which is more convenient for giving detailed proofs and which will be used for the main part of the paper. by

Given a metric $\underline{g}$ on $\mathbb{M}$ as in Section 1.2 and a small positive number $\varepsilon$ define a new metric $\underline{g}^{\varepsilon}$

$$
\begin{aligned}
& \underline{g}_{\mu \nu}^{\varepsilon} d x^{\mu} d x^{\nu}=-p^{\varepsilon}(t, x)^{2} d t^{2}+g_{i j}^{\varepsilon}(t, x) d x^{i} d x^{j}, \\
& p^{\varepsilon}(t, x)=p(\varepsilon t, \varepsilon x), \quad g_{i j}^{\varepsilon}(t, x)=g_{i j}(\varepsilon t, \varepsilon x) .
\end{aligned}
$$

The next theorem describes a class of solutions to $\left(\mathrm{NLW}^{g^{\varepsilon}}\right)$, in the case $\beta(|\phi|)=|\phi|^{p-1}$, which are close to the exact soliton solutions of Lemma 1.2 in a certain sense. As in Theorem 1.3 the soliton parameters evolve, although this time the straight line motion will be replaced by geodesic motion with respect to the background metric (to highest order).

First of all it is necessary to generalise the definitions of the parameter spaces (1.19) to take account of a background metric $\underline{g}^{\varepsilon}$. Given $\lambda=(\omega, \theta, \xi, u) \in C^{1}\left(\mathbb{R} ; \mathbb{R}^{2} \times T \Sigma\right)$ define a spatially 
homogeneous metric $\underline{h}=-q^{2} d t^{2}+h_{i j} d x^{i} d x^{j}$ and corresponding orthonormal frame $B$ by

$$
\begin{aligned}
& q(t)=p(\varepsilon t, \varepsilon \xi(t)), \\
& h_{i j}(t)=g_{i j}(\varepsilon t, \varepsilon \xi(t)), \quad \mathbf{h}(t)=\operatorname{det} h_{i j}(t), \\
& B_{i}^{I}(t)=A_{i}^{I}(\varepsilon t, \varepsilon \xi(t)) \quad \text { so that } h^{i j}(t) B_{i}^{I}(t) B_{j}^{J}(t)=\delta^{I J},
\end{aligned}
$$

where $A$ is as in (1.9). At time $t \in \mathbb{R}$ the parameters take their values in

$$
O^{\underline{g}, t} \equiv\left\{\lambda=(\omega, \theta, \xi, u) \in \mathbb{R}^{2} \times T \Sigma:|\omega|<m \text { and }|u|_{h}<q\right\}
$$

It is also useful to define the stable parameter space by

$$
O_{\text {stab }}^{\underline{g}, t} \equiv O^{\underline{g}, t} \cap\{\omega \in \mathbf{I}\}
$$

and the corresponding space-time definitions are:

$$
\tilde{O}^{\underline{g}} \equiv \bigcup_{t \in \mathbb{R}}\{t\} \times O^{\underline{g}, t} \subset \mathbb{R}^{2} \times T \mathbb{M}, \quad \tilde{O}_{\text {stab }}^{\underline{g}} \equiv \bigcup_{t \in \mathbb{R}}\{t\} \times O_{\text {stab }}^{\underline{g}, t} \subset \mathbb{R}^{2} \times T \mathbb{M}
$$

DEFINITION 1.5 (Unperturbed evolution). - For initial values

$$
\lambda(0)=(\omega(0), \theta(0), \xi(0), u(0)) \in O_{\text {stab }}^{\underline{g}, 0}
$$

the unperturbed evolution, i.e. the zeroth order approximation to the evolution of the parameters $\lambda^{(0)}=\left(\omega^{(0)}, \theta^{(0)}, \xi^{(0)}, u^{(0)}\right)$, is described as follows. Define as in $(1.10)$

$$
q^{(0)}(t)=p^{\varepsilon}\left(t, \xi^{(0)}(t)\right) \quad \text { and } \quad h_{i j}^{(0)}(t)=g_{i j}^{\varepsilon}\left(t, \xi^{(0)}(t)\right)
$$

Then

- $\omega^{(0)}(t)=\omega(0)$ is constant,

- $\xi^{(0)}(t)$ is a solution of the geodesic equation $\left(\mathrm{GEO}^{g^{\varepsilon}}\right)$ with initial values $\xi^{(0)}(0)=\xi(0)$ and $\partial_{t} \xi^{(0)}(0)=u(0)$ and $u^{(0)}(t)=\partial_{t} \xi^{(0)}(t)$ is its velocity,

- $\theta^{(0)}$ is given by $\theta^{(0)}(t)=\int_{0}^{t} \frac{\omega(0) q^{(0)}}{\gamma^{(0)}}+\theta(0)$ with $\gamma^{(0)}=\bar{\gamma}\left(\left(q^{(0)}\right)^{-1}\left|u^{(0)}\right|_{h^{(0)}}\right)$.

For the origin of the last line see (1.24).

Using the conjugate momentum variable $\pi$ in place of $u$ the unperturbed evolution corresponds to integral curves of a simple vector field $\mathbf{V}_{\mathbf{0}}^{*}+\varepsilon \mathbf{V}_{\mathbf{1}}^{*}$ as now described. Recall that the momentum variable $\pi \in T_{\xi}^{*} \Sigma$ was defined in Section 1.4, and correspondingly the geodesic equation $\left(\mathrm{GEO} \underline{g}^{\varepsilon}\right)$ was written as a first order system. Combining this with the highest order evolution 
of $(\omega, \theta)$ in Section 1.6 leads to the following system:

$$
\begin{aligned}
& \frac{d \omega}{d t}=0 \\
& \frac{d \theta}{d t}=\frac{\omega q}{\sqrt{1+|\pi|_{h}^{2}}}, \\
& \frac{d \xi^{j}}{d t}=\frac{q h^{j k}}{\sqrt{1+|\pi|_{h}^{2}}} \pi_{k}, \\
& \frac{d \pi_{k}}{d t}=-\varepsilon \sqrt{1+|\pi|_{h}^{2}} q_{, k}-\frac{\varepsilon q h_{, k}^{j l}}{2 \sqrt{1+|\pi|_{h}^{2}}} \pi_{j} \pi_{l},
\end{aligned}
$$

in which $q, h$ are as in (1.45). Writing these equations all together in terms of the variable $\lambda^{*}=\mathcal{L}(\lambda)=(\omega, \theta, \xi, \pi) \in \mathbb{R}^{2} \times T^{*} \Sigma$ defined previously (see (1.29)) the right hand side defines a vector field on $\mathbb{R}^{2} \times T^{*} \Sigma$ which will be denoted $\mathbf{V}_{\mathbf{0}}^{*}\left(\lambda^{*} ; \underline{h}\right)+\varepsilon \mathbf{V}_{\mathbf{1}}^{*}\left(\lambda^{*} ; \underline{g}^{\varepsilon}, \varepsilon\right)$. The unperturbed evolution $\lambda^{(0)}(t)$ induces a function $\lambda^{*(0)}(t)=\mathcal{L}\left(\lambda^{(0)}(t)\right)$ which satisfies

$$
\frac{d \lambda^{*(0)}}{d t}=\mathbf{V}_{\mathbf{0}}^{*}\left(\lambda^{*(0)} ; \underline{h}\right)+\varepsilon \mathbf{V}_{\mathbf{1}}^{*}\left(\lambda^{*(0)} ; \underline{g}^{\varepsilon}, \varepsilon\right)
$$

Notice that $\mathbf{V}_{1}^{*}$ is $C^{1}$ for a $C^{2}$ metric $\underline{g}$. Using the inverse function $\mathcal{L}^{-1}$ of (1.30) it is useful to introduce

$$
\mathbf{V}_{1} \equiv\left(D_{1} \mathcal{L}^{-1}\left(\mathbf{V}_{1}^{*}\right)+D_{2} \mathcal{L}^{-1}\left(\left(D_{0} \underline{g}\right)^{\varepsilon}+\left(D_{i} \underline{g}\right)^{\varepsilon} u^{i}\right)\right) .
$$

(Here $i=1, \ldots, n$ and the notation $\left(D_{i} \underline{g}\right)^{\varepsilon}$ is meant to indicate $\left(\partial_{X^{i}} \underline{g}\right)(T, X)$ evaluated at $(T, X)=(\varepsilon t, \varepsilon \xi(t))$ while $\left(D_{0} g\right)^{\varepsilon}$ indicates $\left(\partial_{T} g\right)(T, X)$ evaluated at $(T, X)=(\varepsilon t, \varepsilon \xi(t))$.) The unperturbed evolution can now be defined by the integral curves of the vector field $\mathbf{V}_{0}+\varepsilon \mathbf{V}_{1}$ on $\mathbb{R}^{2} \times T \Sigma$. It follows directly from the chain rule (since $\underline{g}$ is assumed $C^{2}$ ) that $\mathbf{V}_{\mathbf{1}}\left(t, \lambda ; \underline{g}^{\varepsilon}\right)$ is a $C^{1}$ function of $t, u, \varepsilon \xi$ and by inspection that

$$
\left|\mathbf{V}_{1}\right| \leqslant \varepsilon c_{1} \quad\left|\partial_{t} \mathbf{V}_{1}\right| \leqslant c \varepsilon\left(1+\left|\partial_{t} \lambda-\mathbf{V}_{0}(\lambda ; \underline{h})\right|+\varepsilon\left|\partial_{t} \lambda\right|\right)
$$

with $c_{1}=c_{1}\left(K_{1}, \gamma\right)$ and $c_{2}=c_{2}\left(K_{2}, \gamma\right)$.

The curve $t \mapsto \lambda^{(0)}(t)$ will be compared with a curve $t \mapsto \lambda(t)$, defined in Section 2.2, which will be used to construct an approximation to $(\phi, \psi)$. The dominant part of the solution will be

$$
\left(\phi_{S}(\cdot, \lambda(t), \underline{h}(t)), \psi_{S}(\cdot, \lambda(t), \underline{h}(t))\right)
$$

with $\underline{h}(t)$ given by (1.45) and $\phi_{S}, \psi_{S}$ as in (1.25). Thus the idea is to freeze the metric coefficients $p, g$ at their values at the point $(t, \xi(t)) \in \mathbb{M}$ and try to build a solution from the corresponding flat space solution. This may be expected to work if the metric is slowly varying compared to the length scale over which the soliton is localised: a hope which is borne out by the next theorem. The norm used for comparison of $\lambda$ and $\lambda^{(0)}$ will be

$$
\tilde{\|} \lambda-\lambda^{(0)} \tilde{\|}_{\varepsilon} \equiv\left|\omega-\omega^{(0)}\right|+\varepsilon\left|\theta-\theta^{(0)}\right|+\varepsilon\left|\xi-\xi^{(0)}\right|+\left|u-u^{(0)}\right| .
$$

Remark. - The $\varepsilon$ scaling in the norm $\tilde{\|} \cdot \tilde{\|}_{\varepsilon}$ means that the soliton appears to move along a geodesic when viewed on large scales; this also corresponds to the scaling in Theorem 1.1. 
THEOREM 1.6 (Special case of main theorem). - Assume $n=3$ and $\beta(|\phi|)=|\phi|^{p-1}$ with $p \in[2,7 / 3)$. Let $\underline{g}^{\varepsilon}$ be a pseudo-Riemannian metric of the form (1.44) where $(p, g)$ satisfy the conditions in Section 1.2. Consider the solution $(\phi(t), \psi(t))$ to $\left(\mathrm{NLW} \underline{g}^{\varepsilon}\right)$ with initial values

$$
(\phi(0), \psi(0))=\left(\phi_{S}(\cdot ; \lambda(0), \underline{h}(0)), \psi_{S}(\cdot ; \lambda(0), \underline{h}(0))\right)
$$

with $\left(\phi_{S}, \psi_{S}\right) \in \mathcal{S}$ as in Lemma 1.2 and where $\lambda(0) \in O_{\text {stab }}^{\underline{g}, 0}$ as defined in (1.47). Let $t \mapsto \lambda^{(0)}(t)$ be the unperturbed evolution described in Definition 1.5. There exist positive numbers

$$
\varepsilon_{*}=\varepsilon_{*}\left(\lambda(0), K_{2}\right), \quad T_{*}=T_{*}\left(\lambda(0), K_{2}\right), \quad c=c\left(\lambda(0), K_{2}\right)
$$

and a function $\lambda \in C^{1}\left(\left[0, \frac{T_{*}}{\varepsilon}\right] ; \mathbb{R}^{2} \times T \Sigma\right)$ such that for all $\varepsilon<\varepsilon_{*}$

$$
\begin{aligned}
& \sup _{0 \leqslant t \leqslant \frac{T_{*}}{\varepsilon}}\left(\tilde{\|} \lambda(t)-\lambda^{(0)}(t) \tilde{\|}_{\varepsilon}+\varepsilon^{-1}|\omega(t)-\omega(0)|\right. \\
& \left.\quad+\left\|\phi(\cdot, t)-\phi_{S}(\cdot, \lambda(t), \underline{h}(t))\right\|_{H^{1}}+\left\|\psi(\cdot, t)-\psi_{S}(\cdot, \lambda(t), \underline{h}(t))\right\|_{L^{2}}\right) \leqslant c \varepsilon
\end{aligned}
$$

where, at each time $t, \underline{h}(t)$ is the spatially homogeneous metric defined by (1.45).

Before giving a more general statement in Theorem 1.7 it is necessary to give a list of hypotheses on the nonlinearity.

\subsection{More general hypotheses and statement of main theorem}

In this section some more general assumptions on the nonlinearity $\mathcal{F}(\phi)=\beta(|\phi|) \phi$ are given which allow the analysis to be carried out leading to the statement of a general theorem. To start with it is necessary that the Cauchy problem be locally well-posed for $(\phi, \psi) \in H^{1} \times L^{2}$ in the sense described below in (WP-1)-(WP-2); in Appendix A.10 conditions on $\mathcal{F}$ are given which ensure this holds. For sub-critical nonlinearities the validity of (WP1)-(WP2) in the cases of interest follows in a straightforward way from the basic estimates for the linear inhomogeneous problem i.e. the energy identity and the Strichartz estimate (an appropriate variable coefficient version of which is given in [25, Corollary 5]). The use of this latter estimate places the solution in some $L_{d t}^{Q}\left(L_{d x}^{R}\right)$ space for appropriate $Q, R$ as in Appendix A.10. This leads us to the following two assumptions: given $(\phi(0), \psi(0)) \in H^{1} \times L^{2}$ there exist

$$
t_{\mathrm{loc}}=t_{\mathrm{loc}}\left(\|(\phi(0), \psi(0))\|_{H^{1} \times L^{2}}\right)>0
$$

and a solution

$$
(\mathrm{WP}-1)(\phi, \psi) \in C^{1}\left(\left[0, t_{\mathrm{loc}}\right] ; L^{2} \times H^{-1}\right) \cap C^{0}\left(\left[0, t_{\mathrm{loc}}\right] ; H^{1} \times L^{2}\right)
$$

which is unique under the additional requirement that $\phi \in L^{Q}\left(\left[0, t_{\mathrm{loc}}\right] ; L^{R}\right)$ for some $Q, R$. Furthermore it is assumed that the Cauchy problem is well posed in the sense that for initial values $(\phi(0), \psi(0))$ and $(\tilde{\phi}(0), \tilde{\psi}(0))$ which are close in $H^{1} \times L^{2}$ the following estimate holds for the unique solutions of (WP-1):

$$
\text { (WP-2) } \sup _{0 \leqslant t \leqslant t_{\text {loc }}}\|(\phi-\tilde{\phi}, \psi-\tilde{\psi})\|_{H^{1} \times L^{2}} \leqslant c\|(\phi(0)-\tilde{\phi}(0), \psi(0)-\tilde{\psi}(0))\|_{H^{1} \times L^{2}}
$$

on the common domain of definition $\left[0, t_{\mathrm{loc}}\right]$. The constant in (WP-2) depends on the constant $K_{2}$ introduced in (M-1)-(M-3) as well as the $H^{1} \times L^{2}$ norms of $(\phi(0), \psi(0))$ and $(\tilde{\phi}(0), \tilde{\psi}(0))$.

The next assumptions pertain mostly to the properties of the solitons and their linear stability. 
(NL-1) $\mathcal{F}$ is a $C^{1}$ function $\mathbb{C} \rightarrow \mathbb{C}$ of the form $\mathcal{F}(\phi)=\beta(|\phi|) \phi$ which is $C^{2}$ for $|\phi| \neq 0$,

$(\mathrm{NL}-2)|\mathcal{F}(\phi)|+\left|\phi \mathcal{F}^{\prime}(\phi)\right| \leqslant \tilde{K}_{1}\left(1+|\phi|^{p}\right)$ with $p \leqslant 1+4 /(n-2)$,

(NL-3) For $\phi_{1}, \phi_{2}$ in $H^{1}$ there exists a number $\tilde{K}_{2}=\tilde{K}_{2}\left(\left\|\phi_{1}\right\|_{H^{1}}+\left\|\phi_{2}\right\|_{H^{1}}\right)$ such that $\left\|\mathcal{F}^{\prime}\left(\phi_{1}\right)-\mathcal{F}^{\prime}\left(\phi_{2}\right)\right\|_{L^{n}} \leqslant \tilde{K}_{2}\left\|\phi_{1}-\phi_{2}\right\|_{H^{1}}$. This is ensured for example if

$$
\left|\mathcal{F}^{\prime \prime}(\phi)\right| \leqslant c\left(1+|\phi|^{p-2}\right)
$$

with $p \in[2, \infty)$ for $n=1,2$ or $p \in\left[2,2+\frac{4-n}{n-2}\right]$ for $n=3,4$.

Remark. - In the case of main interest $n=3$, for the pure power nonlinearity, the stability condition $\mathbf{I} \neq 0$ ensures that $p$ is smaller than $1+4 / 3<3$ and so (NL-3) will then hold.

(R) The nonlinearity $\mathcal{F}(\phi)=\beta(|\phi|) \phi$ is such that for $\omega^{2}<m^{2}$ there exists a unique positive, radial solution $f_{\omega}$ of (1.3) of class $C^{4}$ such that the map

$$
(\lambda, \underline{h}) \mapsto\left(\phi_{S}(\cdot ; \lambda, \underline{h}), \psi_{S}(\cdot ; \lambda, \underline{h})\right) \in H^{k} \times H^{k-1},
$$

defined for a constant coefficient metric $\underline{h}$ and $\lambda \in O^{\underline{h}}$, is continuous for $k=4, C^{1}$ for $k=3$ and $C^{2}$ for $k=2$. Also assume that $f$ and its first four derivatives are exponentially decaying and that the map above has the same regularity with $H^{k}$ replaced by the weighted Sobolev space $H^{k, w}$ (in which Lebesgue measure is replaced by $\left(1+|x|^{2}\right)^{w} d x$ ) for all $w>0$.

(IS) The nonlinearity $\mathcal{F}(\phi)=\beta(|\phi|) \phi$ is such that the conclusions of lemma 1.4 hold with the stability interval I used in the definition of $O_{\text {stab }}^{\underline{h}}$ as in (1.35).

Remarks. - (i) The simplest case for which these two assumptions hold is the pure power $\mathcal{F}(\phi)=|\phi|^{p-1} \phi$ with $p \in(1,1+4 / n)$, in which case the stability interval $\mathbf{I}$ is given in (1.36), first proved in the radial case in [16]. Stability in the general case was proved in [23], in which the condition in (IS) appeared as an interpretation of the stability condition at the linear level. The verification of (R)-(IS) for pure power nonlinearities is explained in detail in [23], in Sections 1.3 .1 and 2.6 respectively.

(ii) The pure power nonlinearities are not very differentiable when $p$ is small so it is useful to have examples of smooth $\mathcal{F}$ for which (R)-(IS) hold. In Sections 1.3.1 and 2.6 of [23] explicit structural conditions on $\mathcal{F}$ are given which imply (IS) and (R). (These arise from $[2,3,18,15,13$, 28] and references therein). Useful examples are of the form

$$
\mathcal{F}(\phi)=\left(\left(|\phi|^{2}+\delta^{2}\right)^{\frac{p-1}{2}}-\delta^{p-1}\right) \phi
$$

for positive small $\delta$. The conditions given in [23] which ensure (R)-(IS) can then be explicitly verified. It is worth noting that for general (non pure power) nonlinearities it may not be possible to determine $\mathbf{I}$ any more explicitly than (1.35).

(iii) The case $n=3, m=1, \mathcal{F}(\phi)=|\phi|^{2} \phi-|\phi|^{5} \phi$ which has been often discussed [10,16,1] does not fit immediately into the present framework but could probably be included with some further work.

(iv) The precise nature of the assumption about weighted Sobolev spaces is not important, and is a consequence of the fact that $f_{\omega}$ and its derivatives are actually exponentially decaying at a rate which is uniform in compact subsets of $\{\omega:|\omega|<m\}$. (This is proved by a maximum principle argument $([2,23])$.)

THEOREM 1.7 (Main theorem). - Assume there are given

(i) a metric $\underline{g}$ on $\mathbb{M}=\mathbb{R}^{1+n}$ which satisfies (M-1)-(M-3) in Section 1.2,

(ii) a nonlinear function $\mathcal{F}: \mathbb{C} \rightarrow \mathbb{C}$ of the form $\mathcal{F}(\phi)=\beta(|\phi|) \phi$, with $\beta: \mathbb{R} \rightarrow \mathbb{R}$ such that (WP1)-(WP2), (NL-1)-(NL-3), (R) and (IS) hold. 
Consider the solution $(\phi(t), \psi(t))$ to the Cauchy problem for $\left(\mathrm{NLW}^{g^{\varepsilon}}\right)$ with data satisfying:

$$
\left|\phi(0)-\phi_{S}\left(\cdot ; \lambda_{0}, \underline{h}(0)\right)\right|_{H^{1}}+\left|\psi(0)-\psi_{S}\left(\cdot ; \lambda_{0}, \underline{h}(0)\right)\right|_{L^{2}} \leqslant \varepsilon
$$

with $\left(\phi_{S}, \psi_{S}\right) \in \mathcal{S}$ as in Lemma 1.2 and where $\lambda_{0} \in O_{\text {stab }}^{g, 0}$ as defined in (1.47). Then there exist $\lambda(0) \in O_{\text {stab }}^{g}$, positive numbers $\varepsilon_{*}=\varepsilon_{*}\left(\lambda(0), K_{2}\right), T_{*}=T_{*}\left(\lambda(0), K_{2}\right), c=c\left(\lambda(0), K_{2}\right)$ and a function $\lambda \in C^{1}\left(\left[0, \frac{T_{*}}{\varepsilon}\right] ; \mathbb{R}^{2} \times T \Sigma\right)$ such that for all $\varepsilon<\varepsilon_{*}$ the estimate (1.55) holds with $t \mapsto \lambda^{(0)}(t)$ the unperturbed evolution described in Definition 1.5 with $\lambda^{(0)}(0)=\lambda(0)$.

This theorem, which implies Theorem 1.6, is proved in Section 2.

\subsection{Notational conventions}

The notation $g$ will be used to indicate the pseudo-Riemannian metric on foliated space-time $\mathbb{M}$ with lapse function $p$ and induced metric on the space-like hypersurfaces $g$, as in Section 1.2. $g^{\varepsilon}$ is the rescaling defined in Section 1.6. Similarly $\underline{h}$ will be a metric on $\mathbb{M}$ with constituent functions $q, h$, and will often be spatially homogeneous and obtained by evaluating $(p, g)$ along a curve as in (1.10) or (1.45). As usual the inner product and norm defined by, say, $h$ will be written $\langle v, w\rangle_{h}$ and $|v|_{h}$, and similarly for $g$.

Let $\operatorname{Met}_{0}^{K}$ be the set of $n \times n$ positive symmetric matrices bounded above by $K^{2}$ and with determinant bounded below by $1 / K^{2 n}$, and let $\mathrm{Met}^{K}$ be the set of pairs $\left(q, h_{i j}\right)$ with $h_{i j} \in \operatorname{Met}_{0}^{K}$ and $q \in \mathbb{R}$ satisfying $1 / K \leqslant q \leqslant K$. Let $\mathcal{M e t}_{0}^{s, K_{s}}$ be the set of $n \times n$ positive symmetric matrix valued functions $g_{i j}$ of $(t, x) \in \mathbb{M}$ whose first $s$ derivatives are bounded by $K_{s}^{2}$ and whose determinant is bounded below by $1 / K_{s}^{2 n}$. Let $\mathcal{M e t}^{s, K_{s}}$ be the set of pairs $p, g_{i j}$ where $g_{i j} \in \mathcal{M e t} t_{0}^{s, K_{s}}$ and where $p$ is a real function whose first $s$ derivatives are bounded everywhere by $K_{s}$ and which is itself bounded below by $1 / K_{s}$.

Throughout this paper write

$$
\bar{\gamma}(s)=\left(1-s^{2}\right)^{-1 / 2} .
$$

For $\varepsilon>0$ introduce scaled space $L_{\varepsilon}^{2}$ on $\Sigma \approx \mathbb{R}^{n}$ by $\|u\|_{L_{\varepsilon}^{2}}^{2}=\varepsilon^{-n} \int|u(x)|^{2} d x$, and a corresponding scaled Sobolev norm (using multi-index notation)

$$
\|u\|_{H_{\varepsilon}^{s}}^{2}=\sum_{|\alpha|=1}^{s} \int_{\Sigma}\left(\varepsilon^{2|\alpha|-n}\left|\nabla_{x}^{\alpha} u(x)\right|^{2}+\varepsilon^{-n}|u(x)|^{2}\right) d x .
$$

In the case $\varepsilon=1$ the subscript $\varepsilon$ will be omitted, i.e. $H_{1}^{s}=H^{s}$.

The following positive number acts as an effective inertial mass for the soliton:

$$
\mathbf{m}_{\boldsymbol{\omega}} \equiv\left(\frac{\left\|\nabla f_{\omega}\right\|_{L^{2}}^{2}}{n}+\omega^{2}\left\|f_{\omega}\right\|_{L^{2}}^{2}\right)
$$

while

$$
\boldsymbol{\mu}_{\boldsymbol{\omega}} \equiv-\frac{\partial}{\partial \omega}\left(\omega\left\|f_{\omega}\right\|_{L^{2}}^{2}\right)
$$

determines stability of the soliton. As a general rule the raised index * will be used to indicate cotangent space variables as in the discussion following Section 1.5, except of course in the case of a differential operator where $*$ means adjoint. Underlining of symbols usually refers to spacetime quantities e.g. $g$ is the pseudo-Riemannian space-time metric while $g$ is the metric induced on a $t=$ constant hypersurface. 


\section{Proof of main theorem}

The steps in the proof of Theorem 1.7 are as follows:

(i) Introduce (in Section 2.1) an ansatz involving $2 n+2$ additional free parameters $\lambda(t) \in \mathbb{R}^{2} \times T \Sigma$ at each time.

(ii) Determine $t \mapsto \lambda(t)$ in such a way as to be able to obtain good estimates. This is done in Section 2.2 using the symplectic orthogonality conditions explained in the discussion surrounding Lemma 1.4.

(iii) Obtain in Sections 2.3 and 2.4 an estimate for an energy-like quantity which is shown to be equivalent to the $H^{1} \times L^{2}$ norm on an appropriate subspace defined in (1.42) for appropriate frequencies satisfying the condition in (1.35).

(iv) Obtain in Lemma 2.9 an improved estimate for the frequency $\omega$ using a conservation law which is a consequence of $S^{1}$ invariance of the original equation.

(v) Finally, in Section 2.6, show that the motion is approximately geodesic on appropriate time intervals and complete the proof.

As a general point the stability of the soliton is determined by the condition $\omega \in \mathbf{I}$ which ensures positivity of certain quantities. This is used in an essential way both in the treatment of $\lambda$ in Section 2.2 and in the energy estimate in Sections 2.3-2.4. Initially at $t=0$ the frequency

$$
\omega(0)=\omega_{0} \in \mathbf{I}
$$

by assumption, but it will have to be proved that $\omega(t)$ remains inside an interval $\left[\omega_{0}-\Omega, \omega_{0}+\Omega\right]$, which is a compact subset of $\mathbf{I}$, at subsequent times. Thus throughout the proof care will be taken to state clearly when it is assumed that $\omega \in\left[\omega_{0}-\Omega, \omega_{0}+\Omega\right]$. The estimate for $\omega(t)$ in Lemma 2.9 will then be used to show that it is possible to find a positive $\Omega$ so that this is true throughout the time of existence of the solution described in the theorem.

A related point is that most of the estimates obtained, in particular in Sections 2.2 and 2.3, are uniform for $\lambda$ in compact subsets of the parameter space (1.19). A convenient way to indicate this is to have constants depending upon $\bar{\gamma}\left(q^{-1}|u|_{h}\right)$ and $\left(m^{2}-\omega^{2}\right)^{-1}$. Many constants in estimates will also depend (in a bounded way) on $\varepsilon t$, since the time dependence of the metric is as a $C^{2}$ function of $\varepsilon t$ and it is useful to make the standing assumption that

$$
0 \leqslant t \leqslant \frac{t_{0}}{\varepsilon}
$$

with $t_{0}$ as in Section 1.2. In this section it is convenient to introduce a slow time variable $T=\varepsilon t$, and by abuse of notation functions of $t$ will be regarded interchangeably as functions of $T$, with the dot notation used for $\partial_{T}$ thus:

$$
\dot{f}=\partial_{T} f=\varepsilon^{-1} \partial_{t} f
$$

The assumption above converts to $T \in\left[0, t_{0}\right]$.

\subsection{The ansatz}

To start with expand the set of dependent variables to include a function

$$
\lambda=(\omega, \theta, \xi, u) \in C^{1}\left(\mathbb{R} ; \mathbb{R}^{2} \times T \Sigma\right),
$$


which will be determined later in Section 2.2. Now decompose the solution as:

$$
\begin{aligned}
& \phi(t, x)=\phi_{S}(Z(t, x) ; \lambda(t), \underline{h}(t))+\varepsilon \tilde{\phi}(t, x), \\
& \psi(t, x)=\psi_{S}(Z(t, x) ; \lambda(t), \underline{h}(t))+\varepsilon \tilde{\psi}(t, x),
\end{aligned}
$$

using definitions (1.45) for $\underline{h}(t)$ etc. and where in addition (1.21)-(1.23) are generalised to allow for time dependent metric coefficients:

$$
\begin{aligned}
& \gamma(t)=\bar{\gamma}\left(|u(t)|_{h(t)} / q(t)\right) \\
& Z^{I}(t, x)=\mathbf{Z}^{I}(x ; \lambda(t), \underline{h}(t)), \\
& \text { where } \mathbf{Z}^{I}(x ; \lambda, \underline{h}) \equiv B_{i}^{I}\left(\gamma P_{u, h}(x-\xi)+Q_{u, h}(x-\xi)\right)^{i}, \\
& \Theta(t, x)=\Theta(x ; \lambda(t), \underline{h}(t)) \quad \text { where } \Theta(x ; \lambda, \underline{h}) \equiv \theta-\frac{\omega}{q} \mathbf{Z}(x ; \lambda, \underline{h}) \cdot(B u) .
\end{aligned}
$$

Here it is to be assumed that at each time $\lambda(t) \in O \frac{g^{\varepsilon}, t}{\text { stab }}$, where $O O_{\text {stab }}^{g^{\varepsilon}, t}$ is as in (1.47). The decomposition (2.3) is underdetermined so far because the function $t \mapsto \lambda(t)$ is still to be chosen; this will be done so as to enforce the requirement that at each time $t$ the pair $(\tilde{\phi}, \tilde{\psi})$ lies in the subspace $\Upsilon_{\lambda(t), \underline{h}(t)}$ defined in (1.42). In Section 2.2 it is shown that this condition implies that $\lambda(t)$ evolves according to a set of ordinary differential equations, which is well-posed under the infinitesimal stability hypothesis, i.e. for $\omega \in \mathbf{I}$ where $\mathbf{I}$ is as in (1.35). Energy estimates are then used to obtain bounds for $(\tilde{\phi}, \tilde{\psi})$ in Section 2.3. The aim will be to show that $\lambda(t)$ does not depart by much from the unperturbed evolution $\lambda^{(0)}(t)$ in a specific sense. For this purpose it is useful to introduce the following sets, for given $\lambda^{(0)}=\left(\omega^{(0)}, \theta^{(0)}, \xi^{(0)}, u^{(0)}\right)$

$$
\begin{aligned}
& \mathbf{K}_{\lambda^{(0)}, l, t} \equiv\left\{\lambda=(\omega, \theta, \xi, u) \in O_{\text {stab }}^{\underline{g}^{\varepsilon}, t}:\left|\omega-\omega^{(0)}\right|+\left|u-u^{(0)}\right|<l\right\}, \\
& \mathbf{K}_{\lambda^{(0)}, l, \varepsilon, t} \equiv\left\{\lambda=(\omega, \theta, \xi, u) \in O O_{\text {stab }}^{g^{\varepsilon}, t}:\left|\omega-\omega^{(0)}\right|+\left|u-u^{(0)}\right|+\varepsilon\left|\xi-\xi^{(0)}\right|<l\right\} .
\end{aligned}
$$

\subsection{Determination of $\lambda(t)$ (modulation theory)}

Rewrite the decomposition (2.3) as

$$
\begin{aligned}
& \phi=\left(f_{\omega}(Z)+\varepsilon v\right) e^{i \Theta}=\phi_{S}+\varepsilon v e^{i \Theta}, \\
& \psi=\left(\left(i \omega \gamma \sqrt{\mathbf{h}} f_{\omega}(Z)-\frac{\gamma \sqrt{\mathbf{h}}}{q} \frac{\partial f_{\omega}}{\partial Z^{I}}(B u)^{I}\right)+\varepsilon w\right) e^{i \Theta}=\psi_{S}+\varepsilon w e^{i \Theta} .
\end{aligned}
$$

For some purposes it will be clearer to use instead of $\lambda(t)$ the variable $\Lambda(t)$ obtained by subtracting off the dominant unperturbed part of the evolution (i.e. that described by (1.24)). Thus write

$$
\Lambda \equiv(\omega, \eta, \alpha, u)
$$

where $\alpha(t)$ and $\eta(t)$ are defined by

$$
\xi(t)=\int_{0}^{t} u(s) d s+\alpha(t),
$$




$$
\Theta(t, x)=\int_{0}^{t} \frac{\omega q}{\gamma}-\frac{\omega}{q} Z \cdot(B u)+\eta .
$$

In accordance with the remark just made this is convenient because

$$
\frac{d \Lambda}{d t}=\frac{d \lambda}{d t}-\mathbf{V}_{\mathbf{0}}(\lambda ; \underline{h})
$$

where $\mathbf{V}_{\mathbf{0}}$ is as in (1.24); it will be proved that $\frac{d \Lambda}{d t}$ is $\mathrm{O}(\varepsilon)$.

In the verification of Lemma 1.2 it turns out that for $\underline{h}$ constant and $\lambda$ evolving according to (1.24) the time derivatives of $Z, \Theta$ are $\partial_{t} Z=-\gamma(B u)$ and $\partial_{t} \Theta=\omega q \gamma$. Thus in the present situation it is convenient to define functions which measure the deviation from these values: so consider the functions $\mu^{0}(t, x), \mu^{I}(t, x)$, for $I=1, \ldots, n$, defined by

$$
\begin{aligned}
& \varepsilon \mu^{I}=\frac{\partial Z^{I}}{\partial t}+\gamma(B u)^{I}, \\
& \varepsilon \mu^{0}=\frac{\partial \Theta}{\partial t}-\omega q \gamma .
\end{aligned}
$$

Explicit expressions for these quantities, which are affine in $\dot{\Lambda}$, are given in Appendix A.4.

Equations for $(v, w)$. Substitution of the ansatz into $\left(\mathrm{NLW}^{g^{\varepsilon}}\right)$, leads to the following system for $(v, w)$ (using notation of (2.2)):

$$
\begin{aligned}
\frac{\partial v}{\partial t}+i\left(\omega q \gamma+\varepsilon \mu_{0}\right) v= & \frac{p^{\varepsilon}}{\sqrt{\mathbf{g}^{\varepsilon}}} w+j_{1}(t, x ; \lambda, \dot{\Lambda}, \underline{h}, \underline{\dot{h}})+f_{1}\left(t, x ; \lambda, \underline{g}^{\varepsilon}, \underline{h}\right) \\
\frac{\partial w}{\partial t}+i\left(\omega q \gamma+\varepsilon \mu_{0}\right) w= & -M v+j_{2}(t, x ; \lambda, \dot{\Lambda}, \underline{h}, \underline{\dot{h}}) \\
& +f_{2}\left(t, x ; \lambda, \underline{g}^{\varepsilon}, \underline{h}\right)+\varepsilon \mathcal{N}\left(f_{\omega}(Z), \varepsilon v, \underline{g}^{\varepsilon}, \varepsilon\right) .
\end{aligned}
$$

Here $M=M\left(\lambda, \underline{g}^{\varepsilon}, \underline{h}\right)$ is a self-adjoint second order linear differential operator, with coefficients depending upon $\lambda, \underline{g}^{\varepsilon}, \underline{h}$, given by

$$
\begin{aligned}
M v= & 0-\Delta_{p^{\varepsilon}, g^{\varepsilon}} v+\frac{\omega^{2} \gamma^{2}}{q^{2}}|u|_{g}^{2} p^{\varepsilon} \sqrt{\mathbf{g}^{\varepsilon}} v \\
& +i \frac{\omega \gamma}{q}\left(u_{j} \frac{\partial}{\partial x^{i}}\left(p^{\varepsilon} \sqrt{\mathbf{g}^{\varepsilon}}\left(g^{\varepsilon}\right)^{i j} v\right)+p^{\varepsilon} \sqrt{\mathbf{g}^{\varepsilon}}\left(g^{\varepsilon}\right)^{l j} u_{j} \frac{\partial v}{\partial x^{l}}\right) \\
& +p^{\varepsilon} \sqrt{\mathbf{g}^{\varepsilon}}\left(m^{2} v-\beta\left(f_{\omega}\right) v-f_{\omega} \beta^{\prime}\left(f_{\omega}\right) \Re v\right)
\end{aligned}
$$

with notation as in (1.15). In (2.12) there are three classes of inhomogeneous term:

- $j_{1}, j_{2}$ arise from the time dependence of $\Lambda$ and $\underline{h}$,

- $f_{1}, f_{2}$ arise because $\underline{h} \neq \underline{g}^{\varepsilon}$, i.e. because the background metric is not spatially homogeneous, and

- $\mathcal{N}$ arises from linearisation of the nonlinear term.

Explicit formulae and properties for these various terms are given in Appendix A.7.

Symplectic orthogonality conditions. To study (2.12) define a linear operator

$$
\tilde{\mathcal{M}}=\tilde{\mathcal{M}}\left(\lambda, \underline{g}^{\varepsilon}, \underline{h}\right)
$$


by

$$
\tilde{\mathcal{M}}(v, w)=\left(-\partial_{t} v-i \omega q \gamma v+\frac{p^{\varepsilon}}{\sqrt{\mathbf{g}^{\varepsilon}}} w,-\partial_{t} w-i \omega q \gamma w-M v\right)
$$

where $M=M\left(\lambda, \underline{g}^{\varepsilon}, \underline{h}\right)$ is as defined in (2.13). To enforce the condition that $(\tilde{\phi}, \tilde{\psi}) \in \Upsilon_{\lambda, \underline{h}}$ which was introduced in the discussion surrounding (1.42), notice its equivalence to the requirement that $(v, w)$ be $L^{2}$ orthogonal (in the usual sense) to the $(2 n+2)$ pairs of functions

$$
e^{-i \Theta} \frac{\partial}{\partial \lambda_{A}}\left(\psi_{S},-\phi_{S}\right)
$$

LEMMA 2.1. - There exist $2 n+2$ pairs $\left\{\left(a_{A}, b_{A}\right)\right\}_{A=-1}^{2 n+1}$ of functions, explicit formulae for which are given in (A.24)-(A.27) in Appendix A.6, which have the same linear span as (2.15). They depend parametrically on $\lambda, \underline{h}$ and the condition $(\tilde{\phi}, \tilde{\psi}) \in \Upsilon_{\lambda, \underline{h}}$ is equivalent to the requirements:

$$
\mathcal{C}_{A}(v(t), w(t) ; \lambda(t), \underline{h}(t))=0
$$

for all $t$ and for $A=-1,2 n+1$, where $\mathcal{C}_{A}$ are linear functionals defined by

$$
\mathcal{C}_{A}(v, w ; \lambda, \underline{h})=\int_{\mathbb{R}^{n}}\left(\left\langle w, b_{A}(Z ; \lambda)\right\rangle_{L^{2}}+\left\langle v, a_{A}(Z ; \lambda)\right\rangle_{L^{2}}\right) d x
$$

with $Z=Z(t, x)$ as in (2.4). Finally, the map $(\lambda, \underline{h}) \mapsto\left(b_{A}(\cdot ; \lambda), a_{A}(\cdot ; \lambda)\right)$ is continuous into $H^{3} \times H^{2}$ for $\lambda \in O^{\underline{h}}$ and $C^{1}$ into $H^{2} \times H^{1}$.

In the case of flat space, when $\underline{h}$ is independent of time and space, the pairs $\left(a_{A}, b_{A}\right)$ lie in the generalised null space of $\tilde{\mathcal{M}}^{*}$, the $L^{2}(d x d t)$ adjoint of $\tilde{\mathcal{M}}$. (See Appendix A.6 and [23] for the Euclidean case.) In the present situation the slow variation of $\Lambda$ and the background metric introduces some $\mathrm{O}(\varepsilon)$ errors, indicated by the following formula:

$$
\tilde{\mathcal{M}}^{*}\left(a_{A}, b_{A}\right)=D_{A B}(\lambda, \underline{h})\left(a_{B}, b_{B}\right)+\varepsilon\left(\mathbf{J}_{A}^{1}, \mathbf{J}_{A}^{2}\right) .
$$

In this formula, which is fully explained and derived in Appendix A.6, $D_{A B}$ is a matrix whose only non-zero entries are $D_{-1,0}$ and $D_{n+i, I}$, while $\mathbf{J}_{A}^{1,2}=\mathbf{J}_{A}^{1,2}\left(Z ; \lambda, \dot{\Lambda}, \underline{h}, \underline{\dot{h}}, \underline{g}^{\varepsilon}, \underline{\dot{g}}^{\varepsilon}, \nabla \underline{g}^{\varepsilon}\right)$ are exponentially decaying functions of $Z$ which are affine in $\dot{\Lambda}, \underline{\dot{\phi}}$; the regularity properties of the $\mathbf{J}$ 's, which are recorded in Lemma A.6 follow directly from assumption (R).

The conditions (2.16) are preserved by the flow if

$$
\int_{\mathbb{R}^{n}}\left(\left\langle a_{A}, j_{1}+f_{1}\right\rangle+\left\langle b_{A}, j_{2}+f_{2}+\varepsilon \mathcal{N}\right\rangle+\varepsilon\left\langle\mathbf{J}_{A}^{1}+i \mu_{0} a_{A}, v\right\rangle+\varepsilon\left\langle\mathbf{J}_{A}^{2}+i \mu_{0} b_{A}, w\right\rangle\right) d x=0
$$

for $A \in\{-1, \ldots, 2 n+1\}$; this set of conditions is to be regarded as a system of equations which implicitly determine $\lambda(t)$.

Now for $\varepsilon$ sufficiently small it is possible to find $\lambda(0)$, close to $\lambda_{0}$, such that at time $t=0$ the condition (2.16) holds: this follows from the implicit function theorem exactly as in [23, Lemma 2.4]. The conditions (2.19) then ensure that (2.16) continues to hold at subsequent times.

Modulation equations for $\lambda(t)$. The aim of the remainder of this section is simply to prove that the conditions (2.19) are equivalent to a system of differential equations which determine 
$\lambda(t)$, and thus to obtain a local existence theorem for a function $t \mapsto \lambda(t)$ such that (2.19) holds. This is done in three lemmas:

- Lemma 2.2 records the result (2.24)-(2.27) of an explicit calculation of the integrals in (2.19) which is carried out in Appendix A.9.

- Lemma 2.3 shows that the equations in Lemma 2.2 determine $\dot{\lambda}$ (or equivalently $\dot{\Lambda}$ ) explicitly and hence gives a system of ODE's for $\lambda$.

- Lemma 2.4 is a local existence theorem for $\lambda$.

Before giving the statement of Lemma 2.2 it is worth recording how the different terms in (2.19) contribute to, and defining some classes of inhomogeneous "error" terms which appear in, the final result (2.24)-(2.27). Also recall the standing assumption (2.1) and the surrounding remarks.

- The integrals involving $j_{1}, j_{2}$ are computed exactly and contribute to the left hand side of (2.24)-(2.27).

- The integrals involving $f_{1}, f_{2}$ are split up into $\mathrm{O}(1)$ terms which contribute to the left hand side of (2.24)-(2.27) and $\mathrm{O}(\varepsilon)$ terms $\varepsilon F$ with $F$ in the class $\mathfrak{E}_{1}$ of $C^{1}$ functions of $(t, \lambda) \in \tilde{O}^{g^{\varepsilon}}$, which satisfy the bound: 6

$$
|F| \leqslant c=c\left(\bar{\gamma}\left(q^{-1}|u|_{h}\right),\left(m^{2}-\omega^{2}\right)^{-1}\right)<+\infty .
$$

In addition this estimate depends on $K_{2}$ - see the final section of the proof in Appendix A.9. These $\mathrm{O}(\varepsilon)$ terms contribute to the right-hand side of (2.24)-(2.27).

- The term involving $\mathcal{N}$ is of the form $\varepsilon F$ where $F$ lies in the class $\mathfrak{E}_{2}$ of continuous functions of $(t, \lambda, v, w) \in \tilde{O}^{g^{\varepsilon}} \times H^{1} \times L^{2}$, which are $C^{1}$ with respect to $(t, \lambda)$ and satisfy the estimate:

$$
|F| \leqslant c=c\left(\bar{\gamma}\left(q^{-1}|u|_{h}\right),\left(m^{2}-\omega^{2}\right)^{-1},\|(v, w)\|_{H^{1} \times L^{2}}\right) .
$$

This estimate also depends upon $K_{0}$ and the inequality assumed in (NL-3).

- The integrals on the second line of (2.19). These are affine in $\dot{\Lambda}$ i.e. of the form $\varepsilon \tilde{F}_{A}$ with

$$
\tilde{F}_{A}=\tilde{F}_{A}^{(0)}\left(t, \lambda ; v, w, \underline{g}^{\varepsilon}, \underline{h}, \varepsilon\right)+\tilde{F}_{A B}\left(t, \lambda ; v, w, \underline{g}^{\varepsilon}, \underline{h}, \varepsilon\right) \dot{\Lambda}_{B}
$$

with $\tilde{F}_{A}^{(0)}$ in the class $\mathfrak{E}_{2}$ just defined (see (2.21)) and $\tilde{F}_{A B}$ are in the class $\mathfrak{E}_{3}$ of continuous functions of $(t, \lambda, v, w) \in \tilde{O}^{\underline{g}^{\varepsilon}} \times L^{2} \times H^{-1}$, which are $C^{1}$ with respect to $(t, \lambda)$, and satisfy

$$
\varepsilon\left|\tilde{F}_{A B}\left(t, \lambda ; v, w, \underline{g}^{\varepsilon}, \underline{h}, \varepsilon\right)\right| \leqslant c\left(\|\varepsilon v\|_{L^{2}}+\|\varepsilon w\|_{H^{-1}}\right)
$$

with $c=c\left(\bar{\gamma}\left(q^{-1}|u|_{h}\right),\left(m^{2}-\omega^{2}\right)^{-1}\right)$. In addition the estimate depends on $K_{1}-$ see Lemma A.6. In the next lemma $T$ is the slow time variable as in (2.2).

LEMMA 2.2. - The conditions (2.19) are equivalent to an implicit system of equations for $\dot{\Lambda}=\partial_{T} \Lambda=\varepsilon^{-1} \partial_{t} \Lambda$ of the form:

$$
\begin{aligned}
& \boldsymbol{\mu}_{\boldsymbol{\omega}}\left(\partial_{T} \eta+\frac{\omega \gamma}{q} u_{j} \partial_{T} \alpha^{j}\right)=\varepsilon \tilde{F}_{-1}\left(t, \lambda ; v, w, \dot{\Lambda}, \underline{g}^{\varepsilon}, \underline{h}, \varepsilon\right), \\
& \boldsymbol{\mu}_{\boldsymbol{\omega}} \partial_{T} \omega=\varepsilon \tilde{F}_{0}\left(t, \lambda ; v, w, \dot{\Lambda}, \underline{g}^{\varepsilon}, \underline{h}, \varepsilon\right) \\
& \sqrt{\mathbf{h}}\left(\partial_{T}\left(\frac{\left\|\nabla f_{\omega}\right\|_{L^{2}}^{2}}{n q} \gamma u_{a}\right)+\omega\left\|f_{\omega}\right\|_{L^{2}}^{2} \partial_{T}\left(\frac{\omega}{q} \gamma u_{a}\right)\right)
\end{aligned}
$$

\footnotetext{
${ }^{6}$ Recall the remark made just prior to Section 2.1.
} 


$$
\begin{aligned}
& +\sqrt{\mathbf{h}} \mathbf{m}_{\omega}\left(\frac{\gamma}{2 q} u_{i} u_{j} h_{, k}^{i j}+\gamma q_{, k}\right)=\varepsilon \tilde{F}_{i}\left(t, \lambda ; v, w, \dot{\Lambda}, \underline{g}^{\varepsilon}, \underline{h}, \varepsilon\right), \\
& \mathbf{m}_{\omega} \partial_{T} \alpha^{j}=\varepsilon \tilde{F}_{n+j}\left(t, \lambda ; v, w, \dot{\Lambda}, \underline{g}^{\varepsilon}, \underline{h}, \varepsilon\right)
\end{aligned}
$$

where the inhomogeneous terms are $C^{1}$ in $(t, \lambda)$ and are as described in (2.22) and the surrounding discussion.

Proof. - The proof of this, which is given in Appendix A.9, consists of a direct computation of the inner products in (2.19) using formulae in Appendices A.6 and A.7. The calculation is carried out at fixed time $t$ under the assumption that $\lambda=\lambda(t)$ lies in a compact subset of parameter space (1.19) so that the dependence of various estimates on $\lambda$ is as in (2.20)-(2.23). (It will be shown in the final stage of the proof of the main theorem that $\lambda(t)$ remains close to the unperturbed evolution $\lambda^{(0)}(t)$ (of Definition 1.5) so that $\lambda(t)$ will certainly remain in such a compact subset throughout the time interval of interest.)

Remark. - Observe that (2.26) gives an evolution equation for the momentum variable $\pi(t)=\boldsymbol{\pi}(u, \underline{h}(t))$ rather than directly for $u$ itself, so that it is in some ways more convenient to use

$$
\lambda^{*}=\mathcal{L}(\lambda(t), \underline{h}(t))
$$

instead of $\lambda(t)$ to describe the soliton.

In the statements of the next two lemmas recall from (2.6) that $(v, w)=e^{-i \Theta}\left(\phi-\phi_{S}, \psi-\psi_{S}\right)$.

LEMMA 2.3. - Let $\Omega>0$ be such that the interval $\left[\omega_{0}-\Omega, \omega_{0}+\Omega\right]$ is a compact subset of $\mathbf{I}$ then there exists a number

$$
\delta^{\sharp}=\delta^{\sharp}\left(K_{1}, \Omega, \omega_{0}, \bar{\gamma}\left(q^{-1}|u|_{h}\right)\right),
$$

which depends continuously upon the final two arguments, such that if $\omega(t) \in\left[\omega_{0}-\Omega, \omega_{0}+\Omega\right]$ and

$$
\left\|\left(\phi(t)-\phi_{S}(\lambda(t), \underline{h}(t)), \psi(t)-\psi_{S}(\lambda(t), \underline{h}(t))\right)\right\|_{L^{2} \times H^{-1}}<\delta^{\sharp}
$$

it is possible to solve (2.24)-(2.27) uniquely for $\dot{\Lambda}$ giving a system of the form:

$$
\dot{\Lambda}=\mathbf{V}_{*}\left(t, \lambda ; v, w, \underline{g}^{\varepsilon}, \underline{h}, \varepsilon\right)=\mathbf{V}_{\mathbf{1}}\left(t, \lambda ; \underline{g}^{\varepsilon}\right)+\varepsilon \mathbf{V}_{\mathbf{2}}\left(t, \lambda ; v, w, \underline{g}^{\varepsilon}, \underline{h}, \varepsilon\right)
$$

(where $\mathbf{V}_{2}$ does not depend upon $\dot{\Lambda}$ ). Furthermore $\mathbf{V}_{2}$ lies in the class $\mathfrak{E}_{2}$ defined in (2.21) and there is an estimate $\left|\dot{\Lambda}-\mathbf{V}_{\mathbf{1}}\right| \leqslant \varepsilon c$ with

$$
\begin{aligned}
c=c\left(K_{2}, \bar{\gamma}\left(q^{-1}|u|_{h}\right), \Omega, \omega_{0},\right. & \|\left(\phi(t)-\phi_{S}(\lambda(t), \underline{h}(t)),\right. \\
& \left.\left.\psi(t)-\psi_{S}(\lambda(t), \underline{h}(t))\right) \|_{H^{1} \times L^{2}}\right) .
\end{aligned}
$$

Remark. - (2.30) implies the following equivalent equation for $\lambda$ :

$$
\partial_{t} \lambda=\mathbf{V}=\mathbf{V}_{\mathbf{0}}+\varepsilon \mathbf{V}_{\mathbf{1}}+\varepsilon^{2} \mathbf{V}_{\mathbf{2}}
$$

with the $\mathbf{V}_{\mathbf{i}}$ having the same arguments as above.

Proof. - To see this clearly it is convenient to diagonalise the left hand side of (2.24)-(2.27): notice that by taking appropriate linear combinations one obtains a system of equations of the form

$$
\Delta(\omega) \partial_{T}\left(\omega, \eta, \alpha, \frac{\gamma u}{q}\right)=V^{\sharp}\left(t, \lambda ; \underline{g}^{\varepsilon}, \underline{h}, \varepsilon\right)+\varepsilon F^{\sharp}\left(t, \lambda ; v, w, \dot{\Lambda}, \underline{g}^{\varepsilon}, \underline{h}, \varepsilon\right) .
$$


In this equation $\Delta(\omega)$ is a diagonal $(2 n+2) \times(2 n+2)$ matrix with first two entries $\boldsymbol{\mu}_{\boldsymbol{\omega}}$ and remaining entries equal to $\mathbf{m}_{\boldsymbol{\omega}}$ (see (1.59)-(1.60)):

$$
\Delta(\omega)=\operatorname{diag}\left(\boldsymbol{\mu}_{\boldsymbol{\omega}}, \boldsymbol{\mu}_{\boldsymbol{\omega}}, \mathbf{m}_{\boldsymbol{\omega}}, \ldots, \mathbf{m}_{\boldsymbol{\omega}}\right)
$$

and

$$
V^{\sharp}(\lambda)=\left(0,0,0,-\left(\frac{\gamma}{2 q} u_{i} u_{j} h_{, k}^{i j}+\gamma q_{, k}\right)\right) .
$$

Observe that $V^{\sharp}$ is just $\mathbf{V}_{1}^{*}$ rewritten in terms of $\lambda$ rather than $\lambda^{*}$ i.e. $V^{\sharp}=\mathbf{V}_{1}^{*} \circ \mathcal{L}_{\underline{h}}$. Finally $F^{\sharp}=\left\{F_{A}^{\sharp}\right\}_{A=-1}^{2 n+1}$, being a linear combination of the $\tilde{F}$ 's of (2.22) can be written:

$$
\left(F^{\sharp}\right)_{A}=\left(F^{\sharp}\right)_{A}^{(0)}\left(t, \lambda ; v, w, \underline{g}^{\varepsilon}, \underline{h}, \varepsilon\right)+\left(F^{\sharp}\right)_{A B}\left(t, \lambda ; v, w, \underline{g}^{\varepsilon}, \underline{h}, \varepsilon\right) \dot{\Lambda}_{B}
$$

with $\left(F^{\sharp}\right)^{(0)}$ and $\left(F^{\sharp}\right)_{A B}$ lying in the class $\mathfrak{E}_{2}$ and

$$
\varepsilon\left|\left(F^{\sharp}\right)_{A B}\left(t, \lambda ; v, w, \underline{g}^{\varepsilon}, \underline{h}, \varepsilon\right)\right| \leqslant c\left(\left\|\phi-\phi_{S}\right\|_{L^{2}}+\left\|\psi-\psi_{S}\right\|_{H^{-1}}\right)
$$

with $c=c\left(K_{1}, \bar{\gamma}\left(q^{-1}|u|_{h},\left(m^{2}-\omega^{2}\right)^{-1}\right)\right.$, depending continuously upon its final two arguments. Now recall that while the mass $\mathbf{m}_{\boldsymbol{\omega}}$ is always positive, for $\omega$ in the stable range $\mathbf{I}$ the numbers $\boldsymbol{\mu}_{\boldsymbol{\omega}}$ are also positive, and uniformly so for $\omega$ in a compact subset of I. It follows immediately that it is possible to solve to get a system

$$
\partial_{T}\left(\omega, \eta, \alpha, \frac{\gamma u}{q}\right)=\mathbf{V}_{1}^{*}\left(t, \lambda^{*} ; \underline{g}^{\varepsilon}, \varepsilon\right)+\varepsilon \mathbf{V}_{2}^{*}\left(t, \lambda^{*} ; v, w, \underline{g}^{\varepsilon}, \varepsilon\right) .
$$

This implies (from (1.28))

$$
\partial_{t} \lambda^{*}=\mathbf{V}_{0}^{*}\left(\lambda^{*} ; \underline{h}\right)+\varepsilon \mathbf{V}_{1}^{*}\left(t, \lambda^{*} ; \underline{g}^{\varepsilon}, \varepsilon\right)+\varepsilon^{2} \mathbf{V}_{2}^{*}\left(t, \lambda^{*} ; v, w, \underline{g}^{\varepsilon}, \varepsilon\right) .
$$

Next substitute for $\underline{h}(t)$ from (1.45) and, with use of (1.30), invert (2.28) to obtain

$$
\lambda(t)=\mathcal{L}^{-1}\left(\lambda^{*}(t), \underline{g}(\varepsilon t, \xi(t))\right) .
$$

The chain rule thus gives (suppressing the arguments for clarity)

$$
\begin{aligned}
\partial_{t} \lambda= & D_{1} \mathcal{L}^{-1}\left(\mathbf{V}_{0}^{*}\right)+\varepsilon\left(D_{1} \mathcal{L}^{-1}\left(\mathbf{V}_{1}^{*}\right)+D_{2} \mathcal{L}^{-1}\left(\left(D_{0} \underline{g}\right)^{\varepsilon}+\left(D_{i} \underline{g}\right)^{\varepsilon} u^{i}\right)\right) \\
& \left.+\varepsilon^{2}\left[D_{1} \mathcal{L}^{-1}\left(\mathbf{V}_{2}^{*}\right)+D_{2} \mathcal{L}^{-1}\left(D_{i} \underline{g}\right)^{\varepsilon} \mathbf{V}_{2}^{* i}\right)\right] \\
= & \mathbf{V}\left(t, \lambda ; v, w, \underline{g}^{\varepsilon}, \varepsilon\right) .
\end{aligned}
$$

Here the notation is as in (1.51). In the second line use has been made of the $i$ th component of (2.37), which reads $\partial_{t} \xi^{i}=u^{i}+\varepsilon^{2} \mathbf{V}_{2}^{* i}$. Now to complete the proof of the theorem notice that in (2.38) $\mathbf{V}_{0}=D_{1} \mathcal{L}^{-1}\left(\mathbf{V}_{0}^{*}\right) \circ \mathcal{L}_{\underline{h}}$ and that the second term on the right hand side is $\varepsilon \mathbf{V}_{1} \circ \mathcal{L}_{\underline{h}}^{-1}$ so that the third term defines $\varepsilon^{2} \mathbf{V}_{2} \circ \mathcal{L}_{\underline{h}}^{-1}$.

Given this it is now a simple matter to apply standard results from ODE theory to obtain a local solution $t \rightarrow \lambda(t)$ lying in the set defined in (2.5), under the smallness assumption (2.29). The only point about which care is needed is in the dependencies of the various constants. In the final stage of the proof the local existence theorem is applied repeatedly in conjunction with the 
estimates to show that the solution does not deviate far from the unpertubed evolution $\lambda^{(0)}(t)$ of Definition 1.5. It is thus useful to state the existence theorem for initial data $\lambda\left(t_{1}\right)$ at $t=t_{1}$ which is close to a point $\lambda^{(0)}$ (which is taken to be $\lambda^{(0)}\left(t_{1}\right)$ in Section 2.6). In the following recall (2.5).

LEMMA 2.4 (Local existence). - Assume

(i) $(\phi, \psi)$ is a solution of $\left(\mathrm{NLW}^{g^{\varepsilon}}\right)$, as in (WP1)-(WP2), and satisfying

$$
\|(\phi, \psi)\|_{H^{1} \times L^{2}} \leqslant N_{0}
$$

$$
\text { for } t_{1} \leqslant t \leqslant t_{1}+t^{\#}
$$

(ii) $\lambda^{(0)}=\left(\omega^{(0)}, \theta^{(0)}, \xi^{(0)}, u^{(0)}\right) \in O_{\text {stab }}^{\underline{g}, t_{1}}$ and $\mathbf{K}_{\lambda^{(0)}, l, t_{1}} \subset O_{\text {stab }}^{\underline{g}, t_{1}}$,

(iii) $\left|\omega^{(0)}-\omega_{0}\right| \leqslant \Omega / 4$ where $\left[\omega_{0}-\Omega, \omega_{0}+\Omega\right]$ is a compact subset of the stability interval $\mathbf{I}$,

(iv) $\lambda\left(t_{1}\right)=\left(\omega\left(t_{1}\right), \theta\left(t_{1}\right), \xi\left(t_{1}\right), u\left(t_{1}\right)\right) \in \mathbf{K}_{\lambda(0), l / 4, t_{1}}$,

(v) $\left(\phi\left(t_{1}\right)-\phi_{S}\left(\lambda\left(t_{1}\right), \underline{h}\left(t_{1}\right)\right), \psi\left(t_{1}\right)-\psi_{S}\left(\lambda\left(t_{1}\right), \underline{h}\left(t_{1}\right)\right)\right) \in \Upsilon_{\lambda\left(t_{1}\right), \underline{h}\left(t_{1}\right)}$.

Then there exists a positive number $\delta_{1}=\delta_{1}\left(K_{1}, \lambda^{(0)}, l, \omega_{0}, \Omega\right)$ such that when

$$
\left\|\left(\phi\left(t_{1}\right)-\phi_{S}\left(\lambda\left(t_{1}\right), \underline{h}\left(t_{1}\right)\right), \psi-\psi_{S}\left(\lambda\left(t_{1}\right), \underline{h}\left(t_{1}\right)\right)\right)\right\|_{L^{2} \times H^{-1}}<\delta_{1} \quad \text { and } \quad l<\delta_{1}
$$

there exist $t_{2}=t_{2}\left(\varepsilon, K_{2}, N_{0}, \lambda^{(0)}, l, \omega_{0}, \Omega\right) \in\left(0, t^{\#}\right]$ and $\lambda \in C^{1}\left(\left[t_{1}, t_{1}+t_{2}\right] ; \mathbf{K}_{\lambda^{(0)}, l, t_{1}}\right)$ satisfying (2.24)-(2.27) and such that

$$
\left(\phi(t)-\phi_{S}(\lambda(t), \underline{h}(t)), \psi(t)-\psi_{S}(\lambda(t), \underline{h}(t))\right) \in \Upsilon_{\lambda(t), \underline{h}(t)} .
$$

Proof. - For the proof the solution $(\phi, \psi)$ is assumed known as in (WP1)-(WP2). Now recall from (2.6) that $(v, w)=e^{-i \Theta}\left(\phi-\phi_{S}, \psi-\psi_{S}\right)$ so that $(v, w)$ depend on $\lambda$ and together with (2.10) Eqs. (2.24)-(2.27) lead to a self-contained system of ordinary differential equations for $\lambda$. Notice however, from (2.22), that $\dot{\Lambda}$ is only determined implicitly; however if the quantities $\left(\phi-\phi_{S}(\lambda),\left(\psi-\psi_{S}(\lambda)\right)\right.$ are small it is possible, by the previous lemma, to solve for $\dot{\Lambda}$, giving a standard system of ODE's for $\left(\Lambda(t), \int_{0}^{t} u(s) d s\right)$, or for $\lambda=(\omega, \theta, \xi, u)$. The aim will be to obtain a time interval for which there is a solution $\lambda(t)$ in the set $\mathbf{K}_{\lambda^{(0)}, l, t_{1}}$. So choose $l$ small enough to ensure that $\lambda \in \mathbf{K}_{\lambda^{(0)}, l, t_{1}}$ implies $\left|\omega-\omega_{0}\right| \leqslant \Omega$, this being possible by assumption (iii). Also recall the quantity $\delta^{\sharp}$ of the previous lemma is continuous in its last two arguments. Therefore it is possible to choose $l$ sufficiently small that $\lambda \in \mathbf{K}_{\lambda^{(0)}, l, t_{1}}$ implies $\delta^{\sharp}$ is bounded below by a number $\delta_{0}=\delta_{0}\left(K_{1}, \omega_{0}, \Omega, \lambda^{(0)}, l\right)$. Since $t \mapsto(\phi(t), \psi(t))$ is continuous into $L^{2} \times H^{-1}$, with modulus of continuity depending upon $N_{0}$, it follows that for $\left|\lambda(t)-\lambda^{(0)}\right| \leqslant l$, with $l$ small, the quantity $\left\|\left(\phi(t)-\phi_{S}(\lambda(t), \underline{h}(t)), \psi(t)-\psi_{S}(\lambda(t), \underline{h}(t))\right)\right\|_{L^{2} \times H^{-1}}$ remains smaller than this number $\delta_{0}$ on some time interval depending also upon $N_{0}$. On this time interval it is thus possible to solve uniquely for $\dot{\Lambda}$ by Lemma 2.3. The result now follows by using (2.10) to substitute $\dot{\Lambda}$ by $\dot{\lambda}$ and then applying the local existence theorem for ordinary differential equations.

In the next two sections energy estimates will be obtained which will eventually allow this local solution to be continued to long but finite times.

\subsection{Energy estimates}

In this section the basic energy estimate which gives long-time control of the deformation pair $(\tilde{\phi}, \tilde{\psi})$ defined in (2.3) is obtained. The parameters $\lambda(t)$, or equivalently $\Lambda(t)$, are now regarded as known and have been chosen such that (2.40) holds. The basis for the estimate obtained is the elementary fact that in the flat case if one linearises about one of the exact solutions described 
in Section 1.5 there is a conservation law for a quadratic energy-like quantity (1.41), which has a positivity property ensured by hypothesis (IS). This quantity is the Hessian of the augmented Hamiltonian (1.34). The results of this and the subsequent section show that in the pseudoRiemannian case an $H^{1} \times L^{2}$ estimate can still be obtained from the related quantity (2.50) on long but finite time intervals. Before giving the energy identity it is necessary to make a decomposition of the inhomogeneous terms as given in (2.46) and define a differential operator (2.49). But first the equations for $(\tilde{\phi}, \tilde{\psi})$ are needed.

Equations for $(\tilde{\phi}, \tilde{\psi})$. Substitute (2.3) into $\left(\mathrm{NLW}^{\underline{g}^{\varepsilon}}\right)$ to obtain a system of the form:

$$
\begin{aligned}
& \partial_{t} \tilde{\phi}=\frac{p^{\varepsilon}}{\sqrt{\mathbf{g}}^{\varepsilon}} \tilde{\psi}+\tilde{j}_{1}(t, x ; \lambda, \dot{\Lambda}, \underline{h}, \underline{\dot{h}}),+\tilde{f}_{1}\left(t, x ; \lambda, \underline{g}^{\varepsilon}, \underline{h}\right), \\
& \partial_{t} \tilde{\psi}=-\tilde{M} \tilde{\phi}+\tilde{j}_{2}(t, x ; \lambda, \dot{\Lambda}, \underline{h}, \underline{\dot{h}})+\tilde{f}_{2}\left(t, x ; \lambda, \underline{g}^{\varepsilon}, \underline{h}\right)+\varepsilon \tilde{\mathcal{N}}\left(\phi_{S}(Z), \varepsilon \tilde{\phi}, \underline{g}^{\varepsilon}, \varepsilon\right) .
\end{aligned}
$$

Here the inhomogeneous terms are just $e^{i \Theta}$ times those in (2.12), where $\Theta$ is as in (2.4), i.e. for $i=1,2$ :

$$
\begin{aligned}
\tilde{j}_{i}(t, x ; \lambda, \dot{\Lambda}, \underline{h}, \underline{\dot{h}})= & e^{i \Theta} j_{i}(t, x ; \lambda, \dot{\Lambda}, \underline{h}, \underline{\dot{h}}), \quad \tilde{f}_{i}\left(t, x ; \lambda, \underline{g}^{\varepsilon}, \underline{h}\right)=e^{i \Theta} f_{i}\left(t, x ; \lambda, \underline{g}^{\varepsilon}, \underline{h}\right), \\
\tilde{\mathcal{N}}\left(\phi_{S}, \varepsilon \tilde{\phi}, \underline{g}^{\varepsilon}, \varepsilon\right)= & \frac{1}{\varepsilon^{2}} p^{\varepsilon} \sqrt{\mathbf{g}^{\varepsilon}}\left\{\beta\left(\left|\phi_{S}+\varepsilon \tilde{\phi}\right|\right)\left(\phi_{S}+\varepsilon \tilde{\phi}\right)-\beta\left(\phi_{S}\right) \phi_{S}\right. \\
& \left.-\varepsilon \beta\left(\phi_{S}\right) \tilde{\phi}-\varepsilon \beta^{\prime}\left(\phi_{S}\right) \frac{\left\langle\phi_{S}, \tilde{\phi}\right\rangle}{\left|\phi_{S}\right|} \phi_{S}\right\}
\end{aligned}
$$

Explicit formulae for the $j$ 's and $f$ 's are in Appendix A.7. Furthermore $\tilde{M}=\tilde{M}\left(\underline{g}^{\varepsilon}, Z, \Theta\right)$ is the differential operator defined by the quadratic form

$$
\begin{aligned}
\langle\tilde{\phi}, \tilde{M} \tilde{\phi}\rangle_{L^{2}}= & \frac{1}{2} \int_{\Sigma} p^{\varepsilon} \sqrt{\mathbf{g}^{\varepsilon}}\left(\left(g^{\varepsilon}\right)^{i j}\left\langle\partial_{i} \tilde{\phi}, \partial_{j} \tilde{\phi}\right\rangle+m^{2}|\tilde{\phi}|^{2}\right. \\
& \left.-\beta\left(\left|\phi_{S}\right|\right)|\tilde{\phi}|^{2}-\beta^{\prime}\left(\left|\phi_{S}\right|\right) \frac{\left\langle\phi_{S}, \tilde{\phi}\right\rangle^{2}}{\left|\phi_{S}\right|}\right) d x .
\end{aligned}
$$

Thus writing $\tilde{M}=\tilde{M}\left(\underline{g}^{\varepsilon}, Z, \Theta\right)$ is intended to indicate that the coefficients of $\tilde{M}$ depend upon $t, x$ through $\underline{g}^{\varepsilon}, Z, \Theta$.

Decomposition of inhomogeneous terms. Since $\lambda(t)$ and $\Lambda(t)$ are now known functions (determined in Section 2.2) it is appropriate to substitute for $\dot{\Lambda}$ in (2.41)-(2.42). First of all apply the chain rule and substitute for $\partial_{T} \xi=\varepsilon^{-1} u+\partial_{T} \alpha$ from (2.30) to obtain the following formula for $\underline{\dot{h}}$ :

$$
\begin{aligned}
\underline{\dot{h}}(t) & =D_{0} \underline{g}^{\varepsilon}+\varepsilon\left\langle\dot{\xi}^{i},\left(D_{i} \underline{g}\right)^{\varepsilon}\right\rangle \quad \text { so that }|\underline{\dot{h}}| \leqslant K_{1}\left(1+\left|\partial_{t} \lambda\right|\right) \\
& =\left(D_{0} \underline{g}\right)^{\varepsilon}+\left\langle u^{i},\left(D_{i} \underline{g}^{\varepsilon}\right\rangle+\varepsilon^{2}\left\langle\mathbf{V}_{2}^{i},\left(D_{i} \underline{g}\right)^{\varepsilon}\right\rangle\right. \\
& =\underline{\dot{h}}^{(0)}(t)+\varepsilon \underline{\dot{h}}^{(1)}(t), \quad \text { where } \underline{\dot{h}}^{(0)}(t) \equiv\left(D_{0} \underline{g}\right)^{\varepsilon}+\left\langle u^{i},\left(D_{i} \underline{g}\right)^{\varepsilon}\right\rangle .
\end{aligned}
$$

(Here the notation being used for partial derivatives of $\underline{g}^{\varepsilon}(t, x)=\underline{g}(\varepsilon t, \varepsilon x)$ is as in (1.51).) Now substituting also for $\dot{\Lambda}$ from (2.30) this induces a decomposition of the inhomogeneous terms:

$$
\tilde{j}_{i}\left(t, x ; \lambda, \dot{\Lambda}, \underline{h}, \underline{\dot{h}}^{(0)}(t)+\varepsilon \underline{\dot{h}}^{(1)}(t)\right)=\tilde{j}_{i}^{(0)}(t, x)+\varepsilon \tilde{j}_{i}^{(1)}(t, x),
$$


where the first term is defined by

$$
\tilde{j}_{i}^{(0)}(t, x)=\tilde{j}_{i}\left(t, x ; \lambda(t), \mathbf{V}_{\mathbf{1}}(t, \lambda(t)), \underline{h}(t), \underline{\dot{h}}^{(0)}(t)\right)
$$

and $\tilde{j}_{i}^{(1)}$ is determined by (2.46). The terms $\tilde{j}_{i}^{(0)}$ and $\tilde{j}_{i}^{(1)}$, which are given explicitly in appendix A.7, will be treated differently in the energy estimate. Observe that since $\mathbf{V}_{\mathbf{1}}$ is $C^{1}$ so are the $\tilde{j}_{i}^{(0)}$. The estimates needed for the $\tilde{j}$ terms are given in the same appendix.

Definition of $\tilde{\boldsymbol{X}}$. Further to the definitions surrounding (2.3) it is convenient to extend, at each time $t$, the velocity $u(t) \in T_{\xi}(t) \Sigma$ to a vector field $\tilde{U}=\tilde{U}^{i}(t, x) \frac{\partial}{\partial x^{i}}$ on $\Sigma$. This is done by the global trivialisation determined by the isomorphism $A$ of (1.45) in the following way:

$$
p^{-1}(\varepsilon t, \varepsilon x) A_{i}^{I}(\varepsilon t, \varepsilon x) \tilde{U}^{i}(t, x)=q^{-1}(t) B_{i}^{I}(t) u^{i}(t) .
$$

Notice that this definition implies that $\left(p^{\varepsilon}\right)^{-1}|\tilde{U}|_{g^{\varepsilon}}=q^{-1}|u|_{h}$ on all of $\Sigma$. Also define the differential operator

$$
\tilde{\boldsymbol{X}} \equiv \partial_{t}-\frac{i \omega p^{\varepsilon}}{\gamma}+\tilde{U}^{i}\left(\nabla_{x}\right)_{i}
$$

Energy estimate for $(\tilde{\phi}, \tilde{\psi})$. The next theorem records an identity which will be used to obtain the required $H^{1} \times L^{2}$ control.

\section{THEOREM 2.5. - Assume}

(i) $(\tilde{\phi}, \tilde{\psi})$ are solutions of the system (2.41)-(2.42) deriving from a solution to $\left(\mathrm{NLW}^{g^{\varepsilon}}\right)$ having regularity as in hypotheses (WP1)-(WP2) of Section 1.7.

(ii) There is given $t^{\dagger}>0$ and $\lambda(t)=(\omega, \theta, \xi, u) \in C^{1}\left(\left[0, t^{\dagger}\right] ; \mathbb{R}^{2} \times T \Sigma\right)$. Define a quadratic form on $H^{1} \times L^{2}$ by

$$
\begin{aligned}
\mathcal{E}\left(\tilde{\phi}, \tilde{\psi} ; \lambda, \underline{g}^{\varepsilon}\right)= & \frac{1}{2} \int\left(\frac{p^{\varepsilon}}{\sqrt{\mathbf{g}^{\varepsilon}}}|\tilde{\psi}|^{2}+\langle\tilde{\phi}, \tilde{M} \tilde{\phi}\rangle\right. \\
& \left.-2 \frac{\omega p^{\varepsilon}}{\bar{\gamma}\left(|u|_{h} / q\right)}\langle\tilde{\psi}, i \tilde{\phi}\rangle+2 \tilde{U}^{i}\left\langle\left(\nabla_{x}\right)_{i} \tilde{\phi}, \tilde{\psi}\right\rangle\right) d x
\end{aligned}
$$

and let $E(t)=\mathcal{E}\left(\tilde{\phi}(t), \tilde{\psi}(t) ; \lambda(t), \underline{g}^{\varepsilon}(t), \underline{h}(t)\right)$ be its evaluation at time $t$. Then for all $t_{1} \leqslant t^{\dagger}$

$$
\begin{aligned}
{[E(t)} & \left.+\left\langle\tilde{\psi}(t), \tilde{j}_{1}^{(0)}+\tilde{f}_{1}\right\rangle_{L^{2}}-\left\langle\tilde{\phi}(t), \tilde{j}_{2}^{(0)}+\tilde{f}_{2}\right\rangle_{L^{2}}\right]_{0}^{t_{1}} \\
= & \int_{0}^{t_{1}} \int_{\Sigma}\left(\left\langle\tilde{\psi}, \tilde{\boldsymbol{X}}\left(\tilde{j}_{1}^{(0)}+\tilde{f}_{1}\right)\right\rangle-\left\langle\tilde{\phi}, \tilde{\boldsymbol{X}}\left(\tilde{j}_{2}^{(0)}+\tilde{f}_{2}\right)\right\rangle\right. \\
& +\varepsilon \partial_{T} \tilde{U}^{i}\left\langle\left(\nabla_{x}\right)_{i} \tilde{\phi}, \tilde{\psi}\right\rangle-\varepsilon \partial_{T}\left(\frac{\omega p^{\varepsilon}}{\gamma}\right)\langle\tilde{\psi}, i \tilde{\phi}\rangle \\
& +\frac{1}{2} \varepsilon \partial_{T}\left(\frac{p^{\varepsilon}}{\sqrt{\mathbf{g}^{\varepsilon}}}\right)|\tilde{\psi}|^{2}+\frac{1}{2}\left(\nabla_{x}\right)_{i}\left(\tilde{U}^{i} \frac{p^{\varepsilon}}{\sqrt{\mathbf{g}^{\varepsilon}}}\right) \mid \tilde{\psi}^{2}-\left(\nabla_{x} \cdot \tilde{U}\right) \frac{p^{\varepsilon}}{\sqrt{\mathbf{g}^{\varepsilon}}|\tilde{\psi}|^{2}} \\
& +\varepsilon\left\langle\tilde{j}_{2}^{(1)}+\mathcal{N}, \frac{p^{\varepsilon}}{\sqrt{\mathbf{g}^{\varepsilon}}} \tilde{\psi}-\frac{\omega p^{\varepsilon}}{\gamma} i \tilde{\phi}+\tilde{U}^{i} \nabla_{i} \tilde{\phi}+\tilde{f}_{1}+\tilde{j}_{1}^{(0)}\right\rangle \\
& +\varepsilon\left\langle\tilde{j}_{1}^{(1)}, \tilde{M} \tilde{\phi}+\frac{\omega p^{\varepsilon}}{\gamma} i \tilde{\psi}-\nabla_{i}\left(\tilde{U}^{i} \tilde{\psi}\right)-\tilde{f}_{2}-\tilde{j}_{2}^{(0)}\right\rangle
\end{aligned}
$$




$$
\left.+\left\langle\tilde{\phi},\left(\partial_{t} \tilde{M}-\frac{i \omega p^{\varepsilon}}{\gamma} \tilde{M}+\nabla_{i}\left(\tilde{U}^{i} \tilde{M}\right)\right) \tilde{\phi}\right\rangle\right) d x d t .
$$

Proof. - A straightforward calculation which is valid for classical solutions yields this identity. For solutions having regularity as in hypotheses (WP1)-(WP2) it also holds as these may be approximated by classical solutions in the corresponding topology.

In order to make use of this identity it will be shown (in the next subsection) that $\mathcal{E}$ is equivalent to the energy norm $H^{1} \times L^{2}$ on the subspace $\Upsilon_{\lambda, \underline{h}}$. Control over $(\tilde{\phi}, \tilde{\psi})$ will then be obtained by means of the following estimate of the right hand side of (2.51).

COROllary 2.6. - Assume that $\|(\varepsilon \tilde{\phi}(t), \varepsilon \tilde{\psi}(t))\|_{H^{1} \times L^{2}} \leqslant M_{*}$ and $\left|\omega(t)-\omega_{0}\right| \leqslant \Omega$ for $0 \leqslant t \leqslant t^{b}$, where $\left[\omega_{0}-\Omega, \omega_{0}+\Omega\right]$ is a compact subset of the stability interval $\mathbf{I}$. Then if $M_{*}$ is sufficiently small on $\left[0, t^{b}\right]$ that (2.30)-(2.31) hold there exists $c_{1}, c_{2}$, with dependences as shown, such that for $t \in\left[0, t^{b}\right]$ and with $\gamma(t)$ as in (2.4),

$$
\begin{aligned}
E(t) \leqslant & E(0)+c_{1}\left(K_{2}, \gamma(t), \omega_{0}, \Omega\right)\|(\tilde{\phi}(t), \tilde{\psi}(t))\|_{H^{1} \times L^{2}} \\
& +c_{1}\left(K_{2}, \gamma(0), \omega_{0}, \Omega\right)\|(\tilde{\phi}(0), \tilde{\psi}(0))\|_{H^{1} \times L^{2}} \\
& +\varepsilon \int_{0}^{t} c_{2}\left(K_{2}, \gamma\left(t^{\prime}\right), \omega_{0}, \Omega,\left\|\left(\tilde{\phi}\left(t^{\prime}\right), \tilde{\psi}\left(t^{\prime}\right)\right)\right\|_{H^{1} \times L^{2}}\right) d t^{\prime} .
\end{aligned}
$$

Remark. - Notice that $E$ is a quadratic quantity in $(\tilde{\phi}(t), \tilde{\psi}(t))$ whereas the second term on the right is of linear growth in $\|(\tilde{\phi}(t), \tilde{\psi}(t))\|_{H^{1} \times L^{2}}$, so the corollary is potentially useful.

Proof of Corollary 2.6. - This result is proved by estimating the right hand side of (2.51) in a straightforward way. Notice that it is necessary to substitute for $\dot{\lambda}$, or equivalently $\dot{\Lambda}$, from Lemma 2.3: the smallness condition on $M_{*}$ is imposed to ensure the applicability of that lemma. The assumption on $\omega$ ensures that $\left(m^{2}-\omega^{2}\right)^{-1}$ is bounded in terms of $\omega_{0}, \Omega$, and so all functions in the classes $\mathfrak{E}_{i}$ are bounded in terms only of $K_{2}, \Omega, \omega_{0}$ and $\gamma(t)$. Lines three through six are handled directly using integration by parts, the Cauchy-Schwarz inequality, substituting for $\dot{\Lambda}$ from (2.30)-(2.31) and using Lemma A.8. The estimate of the first and second lines follows directly from Lemma A.9. For the final line use the form of $\tilde{M}$ given in (2.44): this leads to

- terms arising from application of $\tilde{\boldsymbol{X}}$ to $\phi_{S}$ which can be estimated using Lemma A.12, and

- terms arising from differentiation of the coefficients $p^{\varepsilon} \sqrt{\mathbf{g}^{\varepsilon}}\left(g^{\varepsilon}\right)^{i j}$ and $p^{\varepsilon} \sqrt{\mathbf{g}^{\varepsilon}}$.

All together the final line can be estimated as $\leqslant \varepsilon c\left(K_{2}, \omega_{0}, \Omega, \gamma,\|(\tilde{\phi}, \tilde{\psi})\|_{H^{1} \times L^{2}}\right)$.

\subsection{Positivity of the Hessian}

In order to make use of Corollary 2.6 it is necessary to relate the quantity $\mathcal{E}$ of (2.50) and hence $E$, to the $H^{1} \times L^{2}$-norm. This is done in the next lemma (which depends crucially on hypothesis (IS)). Time dependence is suppressed in both the statement and the proof.

THEOREM 2.7. - Let $\underline{g}=(p, g)$ be a metric in the class Met ${ }^{1, K_{1}}$ defined in Sections 1.2 and 1.8 and define $(q, h)$ as in (1.45). Assume $\left[\omega_{0}-\Omega, \omega_{0}+\Omega\right] \subset \mathbf{I}$, the stability interval, that $\lambda=(\omega, \theta, \xi, u) \in O \mathrm{~S}_{\mathrm{stab}}$ and

$$
\begin{gathered}
\left|\omega-\omega_{0}\right| \leqslant \Omega, \\
\bar{\gamma}\left(q^{-1}|u|_{h}\right)+\left(m^{2}-\omega^{2}\right)^{-1}+\varepsilon|\xi| \leqslant l .
\end{gathered}
$$


There exist positive numbers $\varepsilon_{*}, c_{1}$, depending upon $K_{1}, \omega_{0}, \Omega, l$ such that for $\varepsilon<\varepsilon_{*}$ and all $(\tilde{\phi}, \tilde{\psi}) \in \Upsilon_{\lambda, \underline{h}}$, as defined in (1.42),

$$
\mathcal{E}\left(\tilde{\phi}, \tilde{\psi} ; \lambda, p^{\varepsilon}, g^{\varepsilon}\right) \geqslant c_{1}\left(\|\tilde{\phi}\|_{H^{1}}^{2}+\|\tilde{\psi}\|_{L^{2}}^{2}\right) .
$$

Proof. - First notice that, by Lemma 2.8 below, the result claimed follows (with a different $c_{1}$ ) from the inequality

$$
\mathcal{E}\left(\tilde{\phi}, \tilde{\psi} ; \lambda, p^{\varepsilon}, g^{\varepsilon}\right) \geqslant c_{1}\left(\|\tilde{\phi}\|_{L^{2}}^{2}+\|\tilde{\psi}\|_{L^{2}}^{2}\right)
$$

which will now be proved. Assume to the contrary the existence of sequences of positive numbers $\varepsilon^{\nu} \rightarrow 0$, of metric functions $\underline{g}^{\nu}=\left(p^{\nu}, g^{\nu}\right)$ as in Section 1.2, of functions $\left(\phi^{\nu}, \psi^{\nu}\right)$ satisfying $\left\|\phi^{\nu}\right\|_{L^{2}}^{2}+\left\|\psi^{\nu}\right\|_{L^{2}}^{2}=1$, of points $\lambda^{\nu}=(\omega, \theta, \xi, u)^{\nu} \in O_{\text {stab }}^{\underline{g}}$ satisfying (2.52)-(2.53), such that

$$
\begin{aligned}
& \liminf _{\nu \rightarrow \infty} \mathcal{E}\left(\phi^{\nu}, \psi^{\nu} ; \lambda^{\nu},\left(p^{\nu}\right)^{\varepsilon},\left(g^{\nu}\right)^{\varepsilon}\right) \leqslant 0, \\
& \boldsymbol{\Omega}\left(\left(\phi^{\nu}, \psi^{\nu}\right), \frac{\partial}{\partial \lambda_{A}}\left(\phi_{S}, \psi_{S}\right)\left(x ; \lambda^{\nu}, \underline{h}^{\nu}\right)\right)=0,
\end{aligned}
$$

where $\underline{h}^{\nu}=\underline{g}^{\nu}\left(\varepsilon \xi^{\nu}\right)=\left(q^{\nu}, h^{\nu}\right) \in \operatorname{Met}_{1}^{K}$. By phase invariance assume without loss of generality that $\theta^{\nu}=0$. Notice that Lemma 2.8 ensures that the $\phi^{\nu}$ are uniformly bounded in $H^{1}$ so there is a number $N>0$ such that

$$
\sup _{\nu}\left(\left\|\phi^{\nu}\right\|_{H^{1}}+\left\|\psi^{\nu}\right\|_{L^{2}}\right) \leqslant N .
$$

Apply the translations by $\xi^{\nu}$ to the minimising sequences to give new sequences $\tilde{\lambda}^{\nu}$ and $\left(\tilde{\phi}^{\nu}, \tilde{\psi}^{\nu}\right)$; the norms are unchanged. There exists a relabelled subsequence along which $\varepsilon^{\nu} \xi^{\nu}$ converges to a limit $\Xi$ with $|\Xi| \leqslant l$ and after applying the translations $\tilde{\lambda}^{\nu}$ converges to $\lambda=(\omega, 0,0, u)$ satisfying (2.52)-(2.53). Write $\tilde{p}^{\nu}(x)=p^{\nu}\left(\varepsilon^{\nu} x+\varepsilon^{\nu} \xi^{\nu}\right)$ and similarly for $g$; notice that the frozen values, defined as above by $\underline{\tilde{h}}^{\nu}=\left(\tilde{p}^{\nu}(0), \tilde{g}^{\nu}(0)\right)$ are in fact equal to $\underline{h}^{\nu}$. The Arzela-Ascoli theorem implies that there exists a further subsequence along which $\tilde{p}^{\nu}, \tilde{g}^{\nu}$ converge in $C_{\text {loc }}^{0}$ to a limit metric; $C_{\mathrm{loc}}^{0}$ convergence implies that $\tilde{p}^{\nu}, \tilde{g}^{\nu}$ converge uniformly on compact subsets to the constant values $q=p(\Xi), h=g(\Xi)$; write $\underline{h}=-q^{2} d t^{2}+h$ as usual. Translation invariance of Lebesgue measure implies

$$
\liminf _{\nu \rightarrow \infty} \mathcal{E}\left(\tilde{\phi}^{\nu}, \tilde{\psi}^{\nu} ; \tilde{\lambda}^{\nu}, \tilde{p}^{\nu}, \tilde{g}^{\nu}\right) \leqslant 0
$$

Next consider the functions

$$
\frac{\partial}{\partial \lambda^{A}}\left(\phi_{S}, \psi_{S}\right)\left(x ; \tilde{\lambda}^{\nu}, \underline{h}^{\nu}\right)
$$

it is clear from the formulae in Section 1.5 that as $\nu \rightarrow \infty$ these converge in $C_{\text {loc }}^{0}$ to $\frac{\partial}{\partial \lambda^{A}}\left(\phi_{S}, \psi_{S}\right)(x ; \lambda, \underline{h})$. In fact this convergence is also strong in $L^{2}$ : this follows from the assumption of continuity of the map (1.56) into weighted Sobolev spaces $H^{k, w}$ in hypothesis (R). (Alternatively, and more explicitly, note that the functions (2.55) are linear combinations of the functions $\left(a_{A}, b_{A}\right)$ defined in Appendix A.6, and hence have exponential decrease at infinity uniformly in $\nu$ by [23, Theorem 1.4]). In any case as $\nu \rightarrow \infty$ the functions (2.55) converge strongly in $L^{2}$ so, recalling the definition in (1.42) of the symplectic normal space $\Upsilon_{\lambda, \underline{h}}$, it follows from weak continuity of inner products that there exists a further relabelled subsequence along which $\left(\tilde{\phi}^{\nu}, \tilde{\psi}^{\nu}\right)$ converges weakly in $H^{1} \times L^{2}$ to $(\tilde{\phi}, \tilde{\psi}) \in \Upsilon_{\lambda, \underline{h}}$.

$$
\text { Claim A. }-\tilde{\phi} \not \equiv 0 \text {. }
$$


Proof of Claim A. - Assume that $\tilde{\phi}$ is identically zero. By uniform boundedness in $H^{1}$ of $\tilde{\phi}^{\nu}$ together with Rellich's compactness theorem (e.g. [6, Theorem 6.14]) it may be assumed that the $\tilde{\phi}^{\nu}$ converge to 0 strongly in $L^{2}(K)$ for compact $K$. Next we assert that the hypotheses in Section 1.2 together with the uniform decay of $f_{\omega}(Z)$ ensure that the final two integrals in $\langle\tilde{\phi}, \tilde{M} \tilde{\phi}\rangle$ converge to zero. To see this consider the first of these two terms and notice that if $K$ is compact then

$$
\lim _{\nu \rightarrow \infty} \int_{K} \tilde{p}^{\nu} \sqrt{\tilde{\mathbf{g}}^{\nu}} \beta\left(\left|\phi_{S}\left(\cdot ; \tilde{\lambda}^{\nu}, \underline{h}^{\nu}\right)\right|\right)\left|\tilde{\phi}^{\nu}\right|^{2}=0
$$

because $\tilde{\phi}^{\nu}$ converge to 0 strongly in $L^{2}(K)$ while everything else is uniformly bounded in $L^{\infty}$ (by the hypotheses on the metric and on $\phi_{S}$ ). On the other hand it is possible to choose compact $K$ (independent of $\nu$ ) such that $\sup _{\nu} \int_{K^{c}} \tilde{p}^{\nu} \sqrt{\tilde{\mathbf{g}}^{\nu}} \beta\left(\left|\phi_{S}\left(\cdot ; \tilde{\lambda}^{\nu}, \underline{h}^{\nu}\right)\right|\right)\left|\tilde{\phi}^{\nu}\right|^{2}$ is less than any preassigned value (where $K^{c}$ is the complement of $K$ ) because $\left\|\tilde{\phi}^{\nu}\right\|_{L^{2}} \leqslant 1$ and

$$
\lim _{|x| \rightarrow \infty} \sup _{\nu}\left|\beta\left(\left|\phi_{S}\left(\cdot ; \tilde{\lambda}^{\nu}, \underline{h}^{\nu}\right)\right|\right)\right|=0
$$

by hypothesis (R) (or [23, Theorem 1.4]). This implies the truth of the assertion for the penultimate integral in $\langle\tilde{\phi}, \tilde{M} \tilde{\phi}\rangle$, and the argument for the final term is identical and the assertion is proved. Now given the truth of the assertion it follows immediately that

$$
\liminf \int \frac{\tilde{p}^{\nu}}{\sqrt{\tilde{\mathbf{g}}^{\nu}}}\left|\tilde{\psi}^{\nu}\right|^{2}+\tilde{p}^{\nu} \sqrt{\tilde{\mathbf{g}}^{\nu}}\left(\tilde{g}^{\nu}\right)^{i j}\left\langle\partial_{i} \tilde{\phi}^{\nu}, \partial_{j} \tilde{\phi}^{\nu}\right\rangle+m^{2}\left|\tilde{\phi}^{\nu}\right|^{2}+2\left(\tilde{U}^{\nu}\right)^{i}\left\langle\partial_{i} \tilde{\phi}^{\nu}, \tilde{\psi}\right\rangle \leqslant 0
$$

Now $\left|\tilde{U}^{\nu}\right|_{\tilde{g}}^{2}<\tilde{p}^{2}$ with strict inequality uniformly in $\nu$ by (2.53). Therefore the Cauchy-Schwarz inequality implies that the integral in (2.56) is bounded below by $c\left(\left\|\tilde{\phi}^{\nu}\right\|_{L^{2}}^{2}+\left\|\tilde{\psi}^{\nu}\right\|_{L^{2}}^{2}\right)$ with $c$ independent of $\nu$. Thus (2.56) is impossible since $\left\|\tilde{\phi}^{\nu}\right\|_{L^{2}}^{2}+\left\|\tilde{\psi}^{\nu}\right\|_{L^{2}}^{2}=1$.

Referring now to the infinitesimal stability hypothesis (IS) in Section 1.7, the validity of the lemma follows from the next assertion:

Claim B. $-\mathcal{E}(\tilde{\phi}, \tilde{\psi} ; \lambda, q, h) \leqslant 0$.

Proof of Claim B. - As in the proof of Claim A, the uniform decay of $f_{\omega}$ referred to above makes it possible to take the limit under the integral sign in the last two integrals in (2.44). To show "lower semi-continuity" for the others notice that, writing $\mathcal{E}=\mathcal{E}(\tilde{\phi}, \tilde{\psi} ; \lambda, p, g)$,

$$
\begin{aligned}
2 \mathcal{E} & +\int_{\mathbb{R}^{n}}\left(\beta\left(\left|\phi_{S}\right|\right)|\tilde{\phi}|^{2}+\beta^{\prime}\left(\left|\phi_{S}\right|\right) \frac{\left\langle\phi_{S}, \tilde{\phi}\right\rangle^{2}}{\left|\phi_{S}\right|}\right) d x \\
= & \int_{\mathbb{R}^{n}}\left(\frac{p}{\sqrt{\mathbf{g}}}\left|\tilde{\psi}+\frac{\sqrt{\mathbf{g}}}{p} \tilde{U}^{i} \partial_{i} \tilde{\phi}-\frac{\omega \sqrt{\mathbf{g}}}{\gamma} i \tilde{\phi}\right|^{2}+p \sqrt{\mathbf{g}}\left(g^{i j}\left\langle\partial_{i} \tilde{\phi}, \partial_{j} \tilde{\phi}\right\rangle+m^{2}|\tilde{\phi}|^{2}\right)\right. \\
& \left.-p \sqrt{\mathbf{g}}\left|\frac{\tilde{U}^{i}}{p} \partial_{i} \tilde{\phi}-\frac{\omega}{\gamma} i \tilde{\phi}\right|^{2}\right) d x
\end{aligned}
$$

Write the integrand on the right hand side of (2.57) as $\mathbf{i}(\tilde{\phi}, \tilde{\psi} ; \lambda, p, g)$, and define

$$
I_{R}(\tilde{\phi}, \tilde{\psi} ; \lambda, p, g)=\int_{|x| \leqslant R} \mathbf{i}(\tilde{\phi}, \tilde{\psi} ; \lambda, p, g) d^{n} x
$$


The identity $\bar{\gamma}\left(|\tilde{U}|_{g} / p\right)^{-2}+|\tilde{U}|_{g}^{2} / p^{2}=1$ implies:

$$
-p \sqrt{\mathbf{g}}\left|\frac{\tilde{U}^{i}}{p} \partial_{i} \tilde{\phi}-\frac{\omega}{\gamma} i \phi\right|^{2} \geqslant-p \sqrt{\mathbf{g}}\left(g^{i j}\left\langle\partial_{i} \tilde{\phi}, \partial_{j} \tilde{\phi}\right\rangle+\omega^{2}|\tilde{\phi}|^{2}\right) .
$$

Thus $\mathbf{i}(\tilde{\phi}, \tilde{\psi} ; \lambda, p, g) \geqslant 0$; together with weak convergence of $\tilde{\psi}^{\nu}$ and $\nabla \tilde{\phi}^{\nu}$ in $L^{2}(|x| \leqslant R)$ and the strong convergence of $\tilde{\phi}^{\nu}$ in $L^{2}(|x| \leqslant R)$ this gives

$$
\begin{aligned}
I_{R}(\tilde{\phi}, \tilde{\psi} ; \lambda, q, h) \leqslant & \liminf I_{R}\left(\tilde{\phi}^{\nu}, \tilde{\psi}^{\nu} ; \lambda, q, h\right) \\
\leqslant & \liminf \left(I_{R}\left(\tilde{\phi}^{\nu}, \tilde{\psi}^{\nu} ; \tilde{\lambda}^{\nu}, \tilde{p}^{\nu}, \tilde{g}^{\nu}\right)+I_{R}\left(\tilde{\phi}^{\nu}, \tilde{\psi}^{\nu} ; \lambda, q, h\right)\right. \\
& \left.-I_{R}\left(\tilde{\phi}^{\nu}, \tilde{\psi}^{\nu} ; \tilde{\lambda}^{\nu}, \tilde{p}^{\nu}, \tilde{g}^{\nu}\right)\right) \\
\leqslant & \liminf \left[I_{\infty}\left(\tilde{\phi}^{\nu}, \tilde{\psi}^{\nu} ; \tilde{\lambda}^{\nu}, \tilde{p}^{\nu}, \tilde{g}^{\nu}\right)\right. \\
& \left.+\sup _{\|\phi\|_{H^{1}}+\|\psi\|_{L^{2}}=N}\left|I_{R}\left(\phi, \psi ; \tilde{\lambda}^{\nu}, \tilde{p}^{\nu}, \tilde{g}^{\nu}\right)-I_{R}(\phi, \psi ; \lambda, q, h)\right|\right] .
\end{aligned}
$$

The $C_{\mathrm{loc}}^{0}$ convergence of $p^{\nu}, g^{\nu}$ to $q, h$ implies that the second line has limit zero as $\nu \rightarrow \infty$. Therefore taking supremum over $R$

$$
I_{\infty}(\tilde{\phi}, \tilde{\psi} ; \lambda, q, h) \leqslant \liminf I_{\infty}\left(\tilde{\phi}^{\nu}, \tilde{\psi}^{\nu} ; \tilde{\lambda}^{\nu}, \tilde{p}^{\nu}, \tilde{g}^{\nu}\right)
$$

and so by the remark at the beginning of the proof of Claim B

$$
\mathcal{E}(\tilde{\phi}, \tilde{\psi} ; \lambda, q, h) \leqslant \liminf \mathcal{E}\left(\tilde{\phi}, \tilde{\psi} ; \tilde{\lambda}^{\nu}, \tilde{p}^{\nu}, \tilde{g}^{\nu}\right) \leqslant 0
$$

which completes the proof.

LEMMA 2.8. - Let $\underline{g}=(p, g) \in \mathcal{M e t}^{0, K_{0}}$. If

$$
\gamma=\bar{\gamma}\left(|\tilde{U}|_{g} / p\right)<\infty
$$

there exist $C_{i}=C_{i}\left(\gamma, K_{0}\right)<+\infty$ such that

$$
\|\nabla \tilde{\phi}\|_{L^{2}}^{2}+\left\|\tilde{\psi}+\frac{\sqrt{\mathbf{g}}}{p} \tilde{U}^{i} \partial_{i} \tilde{\phi}-\frac{\omega \sqrt{\mathbf{g}}}{\gamma} \tilde{\phi}\right\|_{L^{2}}^{2} \leqslant C_{1} \mathcal{E}(\tilde{\phi}, \tilde{\psi} ; \lambda, p, g)+C_{2}\|\tilde{\phi}\|_{L^{2}}^{2} .
$$

Proof. - In (2.57) estimate (for any $\theta>0$ )

$$
\left|\frac{\tilde{U}^{i}}{p} \partial_{i} \tilde{\phi}-\frac{\omega}{\gamma} i \tilde{\phi}\right|^{2} \leqslant(1+\theta) \frac{|\tilde{U}|_{g}^{2}}{p^{2}}|\nabla \tilde{\phi}|_{g}^{2}+\left(1+\frac{1}{4 \theta}\right) \frac{\omega^{2}}{\gamma^{2}}|\tilde{\phi}|^{2} .
$$

Since $1<\gamma<\infty$ it follows that $1-\gamma^{-2}=|\tilde{U}|_{g}^{2} / p^{2}<1$ so that choosing $\theta$ sufficiently small that $(1+\theta)\left(1-\gamma^{-2}\right)<1$ the result follows.

\subsection{An improved frequency estimate}

Even in the case of a nontrivial background metric the system $\left(\mathrm{NLW}^{g^{\varepsilon}}\right)$ is invariant under the action of $S^{1}$ by phase rotation. This implies a conservation law by Noether's theorem, a fact which in turn provides a stronger estimate on $\omega$ than that obtained by means of the modulation theory already described. Recall that $\omega(0)=\omega_{0}$. 
LEMMA 2.9. - Let $(\phi, \psi)$ be a solution of $\left(\mathrm{NLW}^{\underline{g}^{\varepsilon}}\right)$ of the form (2.3) with regularity as in (WP1)-(WP2) and assume that $t \mapsto \lambda(t)=(\omega, \theta, \xi, u)(t)$ is a $C^{1}$ function such that $(2.40)$ holds on an interval $0 \leqslant t \leqslant t_{1}$ and that $\omega(0)=\omega_{0} \in \mathbf{I}$. Then there exist positive numbers $\rho=\rho\left(\omega_{0}\right)$ and $\delta_{2}$ such that if $\sup _{t \in\left[0, t_{0}\right]}\|(\varepsilon \tilde{\phi}, \varepsilon \tilde{\psi})\|_{L^{2} \times L^{2}}<\delta_{2}$ on an interval $\left[0, t_{0}\right]$, with $t_{0} \leqslant t_{1}$, then

$$
\sup _{t \in\left[0, t_{0}\right]}\left|\omega(t)-\omega_{0}\right| \leqslant \varepsilon^{2} \rho \sup _{t \in\left[0, t_{0}\right]}\|(\tilde{\phi}, \tilde{\psi})\|_{L^{2} \times L^{2}}^{2} .
$$

Proof. - As just remarked the system has an invariance under the action of the group $S^{1}$ by phase rotation. Corresponding to this invariance is the fact that the quantity

$$
\int\langle\phi, i \psi\rangle d x
$$

is independent of time for solutions of (NLW $\underline{g}$ ) with regularity as in (WP1)-(WP2). Now substitute into this the ansatz (2.3) and use the fact that $(\tilde{\phi}, \tilde{\psi}) \in \Upsilon_{\lambda(t), \underline{h}(t)}$ to deduce that the terms linear in $\tilde{\phi}, \tilde{\psi}$ vanish, and hence using the jacobian identity in Appendix A.1 the quantity

$$
-\omega\left\|f_{\omega}(Z)\right\|_{L_{d Z}^{2}}^{2}+\varepsilon^{2}\langle\tilde{\phi}, i \tilde{\psi}\rangle_{L_{d x}^{2}}
$$

is independent of time. The definition of $\mathbf{I}$ implies that the first term has non-zero derivatives for $\left|\omega-\omega_{0}\right|$ small and implies the estimate (2.61).

\subsection{Completion of the proof of main theorem}

In order to complete the proof it is necessary to compare the evolution of $\lambda(t)$ defined by (2.30) (or equivalently Eqs. (2.24)-(2.27)) with the evolution $\lambda^{(0)}(t)$ introduced in Definition 1.5 in Section 1.6. This is carried out in the norm (1.53). To achieve this it is helpful to use the momentum variable $\pi \in T_{\xi}^{*} \Sigma$ defined in Section 1.4 and the variable

$$
\lambda^{*}=\mathcal{L}(\lambda)=(\omega, \theta, \xi, \pi) \in \mathbb{R}^{2} \times T^{*} \Sigma,
$$

defined just prior to (1.29), in terms of which the unperturbed evolution was written in (1.49). An estimate for $\lambda-\lambda^{(0)}$ in (1.53) will follow from an estimate for

$$
\tilde{\|} \lambda^{*}-\lambda^{*(0)} \tilde{\|}_{\varepsilon} \equiv\left|\omega-\omega^{(0)}\right|+\varepsilon\left|\theta-\theta^{(0)}\right|+\varepsilon\left|\xi-\xi^{(0)}\right|+\left|\pi-\pi^{(0)}\right| .
$$

Now let $\lambda(t)$ be the curve defined by (2.30), consider $\lambda^{*}(t)=\mathcal{L}(\lambda(t))$, and, using (2.7)-(2.10) and (2.2)), (2.37) gives

$$
\frac{d \lambda^{*}}{d t}=\mathbf{V}_{\mathbf{0}}^{*}\left(\lambda^{*} ; \underline{h}\right)+\varepsilon \mathbf{V}_{\mathbf{1}}^{*}\left(\lambda^{*} ; \underline{g}^{\varepsilon}, \varepsilon\right)+\varepsilon^{2} \mathbf{V}_{2}^{*}\left(t, \lambda^{*} ; e^{-i \Theta} \tilde{\phi}, e^{-i \Theta} \tilde{\psi}, \underline{g^{\varepsilon}}, \underline{h}, \varepsilon\right) .
$$

This is possible as long as the smallness condition (2.29) holds. In this case $F^{*}$ is a continuous function of $t, \lambda^{*}$ and $(\tilde{\phi}, \tilde{\psi})$ (the latter being given the strong $H^{1} \times L^{2}$ topology) for $\omega$ in a compact subset $\left[\omega_{0}-\Omega, \omega_{0}+\Omega\right]$ of $\mathbf{I}$. 
Introduce, in addition to $\Omega$, two positive numbers $L_{*}, M_{*}$ and define

$$
t_{*}=\inf \left\{t_{0}>0:\left|\omega\left(t_{0}\right)-\omega_{0}\right|>\Omega \text { or } \frac{1}{\varepsilon} \tilde{\|} \lambda^{*}\left(t_{0}\right)-\lambda^{*(0)}\left(t_{0}\right) \tilde{\|}_{\varepsilon}>L_{*}\right.
$$

$$
\text { or }\left\|\left(\tilde{\phi}\left(t_{0}\right), \tilde{\psi}\left(t_{0}\right)\right)\right\|_{H^{1} \times L^{2}}>M_{*}
$$

or $\lambda^{*}(t)$ cannot be extended as a solution of (2.63) beyond $\left.\left[0, t_{0}\right)\right\}$.

Lemma 2.4 implies the existence of $t_{1}=t_{1}(\varepsilon)>0$ such that there is a local solution to (2.24)(2.27) on $\left[0, t_{1}\right]$ and consequently $t_{*}$ is strictly positive. The plan is to show that $t_{*} \geqslant \frac{T_{*}}{\varepsilon}$, where $T_{*}$ is independent of $\varepsilon$, by a contradiction argument using the fact that

$$
\left|\omega(t)-\omega_{0}\right| \leqslant \Omega, \quad \tilde{\|} \lambda^{*}(t)-\lambda^{*(0)}(t) \tilde{\|}_{\varepsilon} \leqslant L_{*} \varepsilon \quad \text { and } \quad\|(\tilde{\phi}, \tilde{\psi})\|_{H^{1} \times L^{2}} \leqslant M_{*}
$$

on the time interval $\left[0, t_{*}\right)$.

To do this observe that (2.65) implies that (2.53) holds for some $l$ depending upon $\Omega, L_{*}$ and the unperturbed evolution $\lambda^{*(0)}$, which is in turn determined by the initial data $\lambda(0)$. Therefore Corollary 2.6 and Theorem 2.7 together imply that for $t<t_{*}$ the following estimate holds

$$
\|(\tilde{\phi}(t), \tilde{\psi}(t))\|_{H^{1} \times L^{2}}^{2} \leqslant c_{3} \cdot\left(1+M_{*}\right)+\varepsilon c_{4} t
$$

where $c_{3}=c_{3}\left(\lambda(0), K_{2}, \Omega, L_{*}\right)$ and $c_{4}=c_{4}\left(\lambda(0), K_{2}, \Omega, L_{*}, M_{*}\right)$. Now choose $M_{*}$ such that $M_{*}^{2}>c_{3}\left(1+M_{*}\right)$ and let

$$
t_{2}=\frac{M_{*}^{2}-c_{3}\left(1+M_{*}\right)}{\varepsilon c_{4}} .
$$

Next let $\varepsilon$ be chosen to ensure that $\varepsilon\|(\tilde{\phi}(t), \tilde{\psi}(t))\|_{H^{1} \times L^{2}} \leqslant \varepsilon M_{*}$ is small enough that Lemma 2.4 and (2.61) can be applied. The latter will give the first estimate of (2.65) as long as $\varepsilon^{2} \rho M_{*}^{2}<\Omega$. To obtain an estimate for $\lambda^{*}(t)-\lambda^{*(0)}(t)$ notice, from (1.49), that $\mathbf{V}_{\mathbf{1}}{ }^{*}$ is globally Lipschitz if $\omega$ and $\pi$ are bounded. To be precise if (2.65) holds there exists a number $c_{5}=c_{5}\left(K_{2}, \Omega, L_{*}\right)$ such that

$$
\| \mathbf{V}_{1}^{*}\left(\lambda_{1}^{*} ; p, g, \varepsilon\right)-\mathbf{V}_{1}^{*}\left(\lambda_{2}^{*} ; p, g, \varepsilon\right) \tilde{\|}_{\varepsilon} \leqslant \varepsilon c_{5} \tilde{\|} \lambda_{1}^{*}-\lambda_{2}^{*} \tilde{\|}_{\varepsilon} \leqslant \varepsilon^{2} c_{5} L_{*} .
$$

It is now straightforward to compare solutions of (2.63) and of (1.50) which have the same initial data:

Lemma 2.10. - There exists $c_{6}$ depending upon $K_{2}, \lambda(0), \omega_{0}, \Omega, L_{*}, M_{*}$ such that for $t<t_{*}$ :

$$
\tilde{\|} \lambda^{*}(t)-\lambda^{*(0)}(t) \tilde{\|}_{\varepsilon} \leqslant \varepsilon^{2} c_{5} L_{*} t+\varepsilon^{2} c_{6} t
$$

Proof. - Write $\lambda_{\varepsilon}^{*}=(\omega, \varepsilon \theta, \varepsilon \xi, \pi)$ and similarly for $\lambda_{\varepsilon}^{*(0)}$. Then

$$
\begin{aligned}
& \partial_{t} \lambda_{\varepsilon}^{*}=\varepsilon \mathbf{V}_{\mathbf{0}}^{*}+\varepsilon \mathbf{V}_{\mathbf{1}}^{*}+\varepsilon^{2} \mathbf{V}_{\mathbf{2}}^{*}, \\
& \partial_{t} \lambda_{\varepsilon}^{*(0)}=\varepsilon \mathbf{V}_{\mathbf{0}}^{*}+\varepsilon \mathbf{V}_{\mathbf{1}}^{*} .
\end{aligned}
$$

For $t<t_{*}$ (2.31) implies the existence of $c_{6}$ as in the statement so that $\left|\mathbf{V}_{\mathbf{2}}^{*}\right| \leqslant c_{6}$. Recall that the initial data determines $\lambda^{*(0)}$ and hence provides a bound on $\gamma(t)$ as long as $t<t_{*}$ so that 
(2.65) holds. The estimate now follows by inspection since $\xi$ always appears as $\varepsilon \xi$ in $\mathbf{V}_{\mathbf{0}}^{*}, \mathbf{V}_{\mathbf{1}}^{*}$ on account of the scaling $\underline{g}^{\varepsilon}(t, x)=\underline{g}(\varepsilon t, \varepsilon x)$. Finally let

$$
t_{3}=\frac{L_{*}}{\varepsilon\left(c_{5} L_{*}+c_{6}\right)}
$$

and defining $T_{*} / \varepsilon=\min \left\{t_{2}, t_{3}\right\}$ the proof of the theorem is completed.

\section{Proof of Theorem 1.1}

Theorem 1.1 will be deduced from the next result which is obtained directly from Theorem 1.7 by rescaling. Introduce the scaling by $\varepsilon$ of space and time (and also the phase):

$$
X=\varepsilon x, \quad \Xi=\varepsilon \xi, \quad T=\varepsilon t, \quad \tilde{\theta}=\varepsilon \theta .
$$

The metric is now

$$
\frac{1}{\varepsilon^{2}} \underline{g}=\frac{1}{\varepsilon^{2}}\left(-p^{2} d T^{2}+g_{i j} d X^{i} d X^{j}\right)
$$

with $p=p(T, X), g_{i j}=g_{i j}(T, X)$. Under this scaling the system (NLW $\underline{g}$ ) can be conveniently written in the form:

$\left(\mathrm{NLW} \mathrm{g}^{\frac{\underline{g}}{\varepsilon^{2}}}\right)$

$$
\begin{aligned}
& \frac{\partial \phi}{\partial T}=\frac{p}{\sqrt{\mathbf{g}}} \psi, \\
& \frac{\partial \psi}{\partial T}=\Delta_{p, g} \phi-\frac{1}{\varepsilon^{2}} p \sqrt{\mathbf{g}}\left(m^{2} \phi-\beta(|\phi|) \phi\right) .
\end{aligned}
$$

(This involves a scaling by $\varepsilon$ of $\psi$ also. The operator $\Delta_{p, g}$ is now defined with $\nabla_{X}$ in place of $\nabla_{x}$ of course.) Define at time $T$ the spatially homogeneous metric

$$
\underline{h}(T)=-q(T)^{2} d T^{2}+h_{i j}(T) d X^{i} d X^{j}
$$

where

$$
q(T)=p(T, \Xi(T)), \quad h_{i j}(T)=g_{i j}(T, \Xi(T)) .
$$

The rescaling converts the norm $\tilde{\|} \cdot \tilde{\|}_{\varepsilon}$ into the $\varepsilon=1$ case, which will be denoted $\tilde{\|} \cdot \tilde{\|}$. The parameters for the soliton will now be taken to be

$$
\underline{\lambda}(T)=(\omega(T), \tilde{\theta}(T), \Xi(T), u(T)) ;
$$

the unperturbed evolution

$$
\underline{\lambda}^{(0)}(T)=\left(\omega^{(0)}(T), \tilde{\theta}^{(0)}(T), \Xi^{(0)}(T), u^{(0)}(T)\right)
$$

is now described as follows: $T \mapsto \Xi^{(0)}(T)$ is a solution of the geodesic equation (GEO) (with parameter $T$ rather than $t), u^{(0)}(T)=\frac{d \Xi^{(0)}}{d T}, \omega^{(0)}(T)=\omega^{(0)}(0)=\omega_{0}$ and

$$
\tilde{\theta}^{(0)}(T)=\int_{0}^{T} \frac{\omega_{0} q^{(0)}}{\bar{\gamma}\left(\left(q^{(0)}\right)^{-1}\left|u^{(0)}\right|_{h^{(0)}}\right)}+\tilde{\theta}^{(0)}(0),
$$


where

$$
q^{(0)}(T)=p\left(T, \Xi^{(0)}(T)\right), \quad h_{i j}^{(0)}(T)=g_{i j}\left(T, \Xi^{(0)}(T)\right) .
$$

With this change of variables Theorem 1.7 translates into the following:

THEOREM 3.1. - Let g be a pseudo-Riemannian metric of the form (1.44), where $(p, g)$ satisfy the conditions in Section 1.2, and let $\beta$ be as in Section 1.7. Consider the solution $(\phi(t), \psi(t))$ to $\left(\mathrm{NLW} \frac{\underline{g}}{\varepsilon^{2}}\right)$ with initial values

$$
\begin{aligned}
& \phi(0, X)=f_{\omega}(Z(0, X) / \varepsilon) e^{i \Theta(0, X) / \varepsilon} \\
& \psi(0, X)=\frac{1}{\varepsilon}\left(i \omega \gamma \sqrt{\mathbf{h}} f_{\omega}(Z(0, X) / \varepsilon)-\frac{\gamma \sqrt{\mathbf{h}}}{q}(B u) \cdot \nabla_{Z} f_{\omega}(Z(0, X) / \varepsilon)\right) e^{i \Theta(0, X) / \varepsilon}
\end{aligned}
$$

where $Z(T, X)=\mathbf{Z}(X ; \underline{\lambda}(T), \underline{h}(T))$ and $\Theta(T, X)=\mathbf{\Theta}(Z(T, X) ; \underline{\lambda}(T), \underline{h}(T))$ with $\mathbf{Z}, \boldsymbol{\Theta}$ as defined in (2.4). If $\underline{\lambda}(0) \in O$ stab $\underline{\underline{g}, 0}$ there exist positive numbers

$$
\varepsilon_{*}=\varepsilon_{*}\left(\underline{\lambda}(0), K_{2}\right), \quad T_{*}=T_{*}\left(\underline{\lambda}(0), K_{2}\right), \quad c=c\left(\underline{\lambda}(0), K_{2}\right)
$$

and a function $\underline{\lambda} \in C^{1}\left(\left[0, T_{*}\right] ; \mathbb{R}^{2} \times T \Sigma\right)$ such that for all $\varepsilon<\varepsilon_{*}$ and $0 \leqslant T \leqslant T_{*}$ the solution persists and satisfies

$$
\begin{aligned}
& \left(\tilde{\|} \underline{\lambda}(T)-\underline{\lambda}^{(0)}(T) \tilde{\|}+\varepsilon^{-1}\left|\omega(t)-\omega_{0}\right|+\left\|\phi(T, X)-f_{\omega}(Z(T, X) / \varepsilon) e^{i \Theta(T, X) / \varepsilon}\right\|_{H_{\varepsilon}^{1}}\right. \\
& \quad+\varepsilon \| \psi(T, X)-\frac{1}{\varepsilon}\left(i \omega \gamma \sqrt{\mathbf{h}} f_{\omega}(Z(T, X) / \varepsilon)\right. \\
& \left.\left.\quad-\frac{\gamma \sqrt{\mathbf{h}}}{q}(B u) \cdot \nabla_{Z} f_{\omega}(Z(T, X) / \varepsilon)\right) e^{i \Theta(T, X) / \varepsilon} \|_{L_{\varepsilon}^{2}}\right) \leqslant c \varepsilon .
\end{aligned}
$$

The statement in this theorem is now very close to that in Theorem 1.1. There are two things remaining to be done to complete the proof of Theorem 1.1:

- Obtain the slightly improved estimate for $\eta$ in (1.13).

- Obtain the estimate for $\partial_{t}\left(\phi-\phi_{0}^{\varepsilon}\right)$ in (1.14).

Introduce, as in Section 2.2, the alternate variable

$$
\underline{\Lambda}=(\omega, \tilde{\eta}, \tilde{\alpha}, u)
$$

with $\tilde{\eta}=\varepsilon \eta$ and $\tilde{\alpha}=\varepsilon \alpha$, so that $\frac{d \underline{\Lambda}}{d \bar{T}}=\frac{d \underline{\lambda}}{d \bar{t}}-V_{0}(\underline{\lambda} ; \underline{h})$. It follows from the formulae in Lemma 2.2 that

$$
\left|\partial_{T} \tilde{\alpha}\right|+\left|\partial_{T} \tilde{\eta}\right| \leqslant c \varepsilon^{2}
$$

from which follows the estimate for $\eta$ as required.

Now to derive the estimate for $\partial_{t}\left(\phi-\phi_{0}^{\varepsilon}\right)$ in (1.14) notice firstly that if $\tilde{\alpha}=0$ the function

$$
\phi_{0}^{\varepsilon}(T, X)=f_{\omega}(Z(T, X) / \varepsilon) e^{i \Theta(T, X) / \varepsilon}
$$

is exactly the function defined in (1.12) (after relabelling $(T, X)$ to $(t, x)$ etc.) By (3.4) the presence of a non-zero $\tilde{\alpha}$ contributes an $\mathrm{O}(\varepsilon)$ error. Next compute

$$
\partial_{T} \phi_{0}^{\varepsilon}(T, X)=\frac{1}{\varepsilon}\left(i \partial_{T} \Theta f_{\omega}(Z(T, X) / \varepsilon)+\partial_{T} Z \cdot \nabla_{Z} f_{\omega}(Z(T, X) / \varepsilon)\right) e^{i \Theta(T, X) / \varepsilon}
$$


Therefore, suppressing the arguments $(T, X)$ of $Z$ and $\Theta$ :

$$
\begin{aligned}
\varepsilon \partial_{T}\left(\phi-\phi_{0}^{\varepsilon}\right)= & \varepsilon \frac{p}{\sqrt{\mathbf{g}}}\left(\psi-\frac{1}{\varepsilon}\left(i \omega \gamma \sqrt{\mathbf{h}} f_{\omega}(Z / \varepsilon)-\frac{\gamma \sqrt{\mathbf{h}}}{q}(B u) \cdot \nabla_{Z} f_{\omega}(Z / \varepsilon)\right) e^{i \Theta / \varepsilon}\right) \\
& -\varepsilon\left(\partial_{T} \phi_{0}^{\varepsilon}-\frac{p}{\sqrt{\mathbf{g}}} \frac{1}{\varepsilon}\left(i \omega \gamma \sqrt{\mathbf{h}} f_{\omega}(Z / \varepsilon)-\frac{\gamma \sqrt{\mathbf{h}}}{q}(B u) \cdot \nabla_{Z} f_{\omega}(Z / \varepsilon)\right) e^{i \Theta / \varepsilon}\right) .
\end{aligned}
$$

To prove Theorem 1.1 it is necessary to estimate this in $L_{\varepsilon}^{2}$. The first line is estimated from Theorem 3.1, so it remains to deal with the second line. As in Appendix A.4 the following formulae hold:

$$
\begin{aligned}
& \frac{\partial Z^{I}}{\partial T}=-\gamma(B u)^{I}-B_{i}^{I}\left(\gamma P_{j}^{i}+Q_{j}^{i}\right) \partial_{T} \tilde{\alpha}^{j}+\mu_{J}^{I} Z^{J}, \\
& \frac{\partial \Theta}{\partial T}=-\omega q \gamma+\partial_{T} \tilde{\eta}-\frac{\omega}{q}(B u)^{I} \tilde{\mu}^{I}+\mu_{J}^{0} Z^{J}
\end{aligned}
$$

for $\mu_{J}^{I}=\mu_{J}^{I}\left(\lambda, \partial_{T} \Lambda, \underline{h}, \partial_{T} \underline{h}\right)$ and $\mu_{J}^{0}=\mu_{J}^{0}\left(\lambda, \partial_{T} \Lambda, \underline{h}, \partial_{T} \underline{h}\right)$ and $\tilde{\mu}^{I}$ as in Appendix A.4. Substitute (3.5) into the second line of (3.6) using (3.7). Terms arising from the first terms in (3.7) cancel with

$$
\frac{p}{\sqrt{g}}\left(i \omega \gamma \sqrt{\mathbf{h}} f_{\omega}(Z / \varepsilon)-\frac{\gamma \sqrt{\mathbf{h}}}{q}(B u) \cdot \nabla_{Z} f_{\omega}(Z / \varepsilon)\right)
$$

up to an error which is $\leqslant c \varepsilon$ by Lemma A.10. The estimate (3.4) makes it easy to estimate expressions arising from the middle terms. The third terms are $\leqslant c \varepsilon$ since $Z / \varepsilon$ times $f_{\omega}(Z / \varepsilon)$ and its derivatives are bounded in $L_{\varepsilon}^{2}$ by the known exponential decay of these functions: this completes the estimation of (3.6) needed. Thus Theorem 1.1 is proved for the general class of nonlinearities described in Section 1.7.

\section{Acknowledgements}

The author is supported by EPSRC grant AF/98/2492. I thank the referee for some comments and corrections.

\section{Appendix A}

\section{A.1. Various formulae}

Let $t$ be fixed and suppressed and let $\gamma=\bar{\gamma}\left(|u|_{h} / q\right)$. The inverse of the function $x \mapsto Z=$ $\mathbf{Z}(x ; \lambda, \underline{h})$, defined in (2.4) is given by

$$
x=\mathbf{x}(Z ; \lambda, \underline{h})=\left(\frac{1}{\gamma} P_{u, h}\left(B^{-1} Z\right)+Q_{u, h}\left(B^{-1} Z\right)\right) .
$$

The Jacobian of the transformation $x \mapsto Z(x)$ is $\gamma \sqrt{\mathbf{h}}$, so that $d^{n} Z=\gamma \sqrt{\mathbf{h}} d^{n} x$. The measures $d^{n} x, d^{n} Z$ will usually be written $d x, d Z$ respectively.

\section{A.2. Projection operators}

Given an inner product $h=h_{i j}$ on $\mathbb{R}^{n}$ it is possible to introduce projection operators in the direction of $u$ and orthogonal to $u$, for any $u \in \mathbb{R}$. These are given by 


$$
\begin{aligned}
& \left(P_{u, h} V\right)^{i}=\frac{\langle u, V\rangle_{h} u^{i}}{|u|_{h}^{2}}, \\
& \left(Q_{u, h} V\right)^{i}=V^{i}-\left(P_{u, h} V\right)^{i}=\frac{|u|_{h}^{2} V^{i}-\langle u, V\rangle_{h} u^{i}}{|u|_{h}^{2}} .
\end{aligned}
$$

A convenient way to write this is to write the isomorphism induced by $h$ :

$$
\begin{aligned}
& \mathbb{R}^{n} \rightarrow\left(\mathbb{R}^{n}\right)^{*}, \\
& V^{i} \mapsto V_{i}=h_{i j} V^{j},
\end{aligned}
$$

in terms of raising/lowering indices in the usual way. The projection operators can then be written

$$
\left(P_{u, h}\right)_{j}^{i}=\frac{u^{i} u_{j}}{|u|_{h}^{2}}, \quad\left(Q_{u, h}\right)_{j}^{i}=\delta_{j}^{i}-\frac{u^{i} u_{j}}{|u|_{h}^{2}}
$$

while indices are raised and lowered with $h$ in the standard way; thus, for example:

$$
\left(Q_{u, h}\right)_{i j}=h_{i j}-\frac{u_{i} u_{j}}{|u|_{h}^{2}}
$$

\section{A.3. Spatial derivatives}

Let $t$ be fixed, and so suppressed, and let $\gamma=\bar{\gamma}\left(|u|_{h} / q\right)$ where $\underline{h}=(q, h)$ is a spatially homogeneous metric (as in (1.45)) with $q, h_{i j}$ depending only on $t$. Then the derivatives of the functions $Z=\mathbf{Z}(x ; \lambda, \underline{h})$ and $\Theta=\mathbf{\Theta}(x ; \lambda, \underline{h})$ defined in (2.4) with respect to $x$ are

$$
\begin{aligned}
\frac{\partial Z^{I}}{\partial x^{j}} & =B_{i}^{I}\left(\gamma\left(P_{u, h}\right)_{j}^{i}+\left(Q_{u, h}\right)_{j}^{i}\right) \quad\left(\text { so that }(B u)^{I} \frac{\partial Z^{I}}{\partial x^{j}}=\gamma u_{j}\right), \\
\frac{\partial \Theta}{\partial x^{j}} & =-\frac{\omega u_{j} \gamma}{q} .
\end{aligned}
$$

\section{A.4. Time derivatives}

Assume that $\underline{h}=(q, h)$ is a spatially homogeneous metric (as in (1.45)) with $q, h_{i j}$ depending only on $t$. The derivatives with respect to $t$ of the functions defined in (2.4) can be computed as (using $\langle$,$\rangle for the duality pairing):$

$$
\begin{aligned}
\partial_{t} Z & =\left\langle\partial_{\lambda} \mathbf{Z}, \partial_{t} \lambda\right\rangle+\left\langle\partial_{\underline{h}} \mathbf{Z}, \partial_{t} \underline{h}\right\rangle \\
& =\left\langle\partial_{\lambda} \mathbf{Z}, V(\lambda)\right\rangle+\left\langle\partial_{\lambda} \mathbf{Z}, \partial_{t} \lambda\right\rangle+\left\langle\partial_{\underline{h}} \mathbf{Z}, \partial_{t} \underline{h}\right\rangle \\
& =-\gamma(B u)+\left\langle\partial_{\lambda} \mathbf{Z}, \partial_{t} \lambda\right\rangle+\left\langle\partial_{\underline{h}} \mathbf{Z}, \partial_{t} \underline{h}\right\rangle,
\end{aligned}
$$

and similarly for $\Theta$. Thus, using the notation of (2.2), the expressions $\mu^{I}, \mu^{0}$ defined in (2.11) can be written in terms of $\mathbf{Z}, \boldsymbol{\Theta}$ defined in (2.4):

$$
\begin{aligned}
& \varepsilon \mu^{I}(t, x)=\varepsilon \boldsymbol{\mu}^{I}(x, \lambda(t), \dot{\Lambda}(t), \underline{h}(t), \underline{\dot{h}}(t)) \quad \text { where } \\
& \boldsymbol{\mu}^{I}(x, \lambda, \dot{\Lambda}, \underline{h}, \underline{\dot{h}})=\left\langle\dot{\Lambda}, D_{\lambda} \mathbf{Z}(x, \lambda, \underline{h})\right\rangle+\left\langle\underline{\dot{h}}, D_{\underline{h}} \mathbf{Z}(x, \lambda, \underline{h})\right\rangle, \\
& \varepsilon \mu^{0}(t, x)=\varepsilon \boldsymbol{\mu}^{0}(x, \lambda(t), \dot{\Lambda}(t), \underline{h}(t), \underline{\dot{h}}(t)) \quad \text { where } \\
& \boldsymbol{\mu}^{0}(x, \lambda, \dot{\Lambda}, \underline{h}, \underline{\dot{h}})=\boldsymbol{\mu}^{I} \frac{\partial \boldsymbol{\Theta}}{\partial Z^{I}}+\left\langle\dot{\Lambda}, D_{\lambda} \boldsymbol{\Theta}(\mathbf{Z}, \lambda, \underline{h})\right\rangle+\left\langle\underline{\dot{h}}, D_{\underline{h}} \boldsymbol{\Theta}(\mathbf{Z}, \lambda, \underline{h})\right\rangle .
\end{aligned}
$$


Here it is to be understood that all quantities are to be evaluated at $\mathbf{Z}(x, \lambda, \underline{h})$. Rewrite all of these as functions of $Z$ by composing with the inverse function (A.1):

$$
\begin{aligned}
& \bar{\mu}^{I}(t, Z)=\mu^{I}(t, \mathbf{x}(Z, \lambda(t), \underline{h}(t))), \quad \bar{\mu}^{0}(t, Z)=\mu^{0}(t, \mathbf{x}(Z, \lambda(t), \underline{h}(t))), \\
& \overline{\boldsymbol{\mu}}^{I}(Z, \lambda, \dot{\Lambda}, \underline{h}, \underline{\dot{h}})=\boldsymbol{\mu}^{I}(\mathbf{x}(Z, \lambda, \underline{h}), \lambda, \dot{\Lambda}, \underline{h}, \underline{\dot{h}}), \\
& \overline{\boldsymbol{\mu}}^{0}(Z, \lambda, \dot{\Lambda}, \underline{h}, \underline{\dot{h}})=\boldsymbol{\mu}^{0}(\mathbf{x}(Z, \lambda, \underline{h}), \lambda, \dot{\Lambda}, \underline{h}, \underline{\dot{h}}) .
\end{aligned}
$$

These are affine in $Z$ : decompose $\bar{\mu}^{I}$ and $\bar{\mu}^{0}$ into parts $\tilde{\mu}^{I}, \tilde{\mu}^{0}$ independent of $Z$ and parts $\mu_{J}^{I} Z^{J}, \mu_{J}^{0} Z^{J}$ linear in $Z$. The following identities summarize these definitions:

$$
\begin{aligned}
& \frac{\partial Z^{I}}{\partial t}=-\gamma(B u)^{I}-\varepsilon B_{i}^{I}\left(\gamma P_{j}^{i}+Q_{j}^{i}\right) \partial_{T} \alpha^{j}+\varepsilon \mu_{J}^{I} Z^{J} \\
& \frac{\partial \Theta}{\partial t}=\omega q \gamma+\varepsilon \partial_{T} \eta-\varepsilon \frac{\omega}{q}(B u)^{I} \tilde{\mu}^{I}+\varepsilon \mu_{J}^{0} Z^{J} .
\end{aligned}
$$

Notice that the derivatives with respect to $\underline{h}$ contribute only to the parts linear in $Z$. The parts independent of $Z$ are given by

$$
\begin{aligned}
& \tilde{\mu}^{0}=\partial_{T} \eta-\frac{\omega}{q}(B u)^{I} \tilde{\mu}^{I}, \\
& \tilde{\mu}^{I}=-B_{i}^{I}\left(\gamma P_{j}^{i}+Q_{j}^{i}\right) \partial_{T} \alpha^{j} .
\end{aligned}
$$

The explicit formulae for the parts linear in $Z \mu_{J}^{I}=\mu_{J}^{I}(\lambda, \dot{\Lambda}, \underline{h}, \underline{\dot{h}})$ and $\mu_{J}^{0}=\mu_{J}^{0}(\lambda, \dot{\Lambda}, \underline{h}, \underline{\dot{h}})$ will not be needed, only the following identities

LEMMA A.1. - With the summation convention

$$
\begin{aligned}
& \mu_{I}^{I}=\frac{\partial \mu^{I}}{\partial Z^{I}}=\frac{\partial_{T}(\gamma \sqrt{\mathbf{h}})}{\gamma \sqrt{\mathbf{h}}}, \\
& (B u)^{J} \mu_{L}^{J}(B(\gamma P+Q))_{i}^{L}=\left(\gamma u_{i}\right)_{t}-\left(\partial_{t}(B u)^{J}\right)(B(\gamma P+Q))_{i}^{J}, \\
& (B(\gamma P+Q))_{i}^{L} \frac{\partial \mu^{0}}{\partial Z^{L}}=-\partial_{t}\left(\frac{\omega \gamma u_{i}}{q}\right) .
\end{aligned}
$$

Proof. - The first is best proved by use of the change of variables for integrals exactly as in [23, Lemma A.2]. The other two are simple calculations.

LEMmA A.2. - Let $\lambda \in \mathbf{K}_{\lambda^{(0)}, l, t}$, the set defined in 2.5 and assume $\underline{h}(t) \in \mathrm{Met}^{K}$ for some $K$. There exists $c=c\left(K, \lambda^{(0)}, l\right)$, uniform for $\lambda^{(0)}$ in a compact subset of the parameter space (1.46), such that

$$
\left|\overline{\boldsymbol{\mu}}^{I}(Z, \lambda, \dot{\Lambda}, \underline{h}, \underline{\underline{h}})\right|+\left|\overline{\boldsymbol{\mu}}^{0}(Z, \lambda, \dot{\Lambda}, \underline{h}, \underline{\dot{h}})\right| \leqslant c(1+|Z|)(|\dot{\Lambda}|+|\underline{\dot{h}}|) .
$$

\section{A.5. Derivatives with respect to velocity $u$}

In order to compute the elements of the generalised null space of the linearised operator it is helpful to first introduce some notation for the derivatives with respect to $u$ of the function $\mathbf{Z}(x ; \lambda, \underline{h})$ defined in (2.4). Let $\gamma=\bar{\gamma}\left(|u|_{h} / q\right)$ and given the expression

$$
Z=\mathbf{Z}^{I}(x ; \lambda, \underline{h})=B_{i}^{I}\left(\gamma\left(P_{u, h}\right)_{j}^{i}(x-\xi)^{j}+\left(Q_{u, h}\right)_{j}^{i}(x-\xi)^{j}\right),
$$


define $\zeta_{i}^{a}=\zeta_{i}^{a}(Z ; \lambda, \underline{h})$ by:

$$
\begin{aligned}
\zeta_{i}^{a} & =\frac{\partial}{\partial u^{i}}\left(\gamma\left(P_{u, h}\right)_{b}^{a}(x-\xi)^{b}+\left(Q_{u, h}\right)_{b}^{a}(x-\xi)^{b}\right) \\
& =\frac{\partial}{\partial u^{i}}\left((\gamma-1)\left(P_{u, h}\right)_{b}^{a}(x-\xi)^{b}+(x-\xi)^{a}\right) \\
& =\frac{\gamma^{2} u_{i}}{q^{2}}\left(P_{u, h}\left(B^{-1} Z\right)\right)^{a}+\frac{\gamma-1}{\gamma|u|_{h}^{2}}\left(Q_{u, h}\right)_{i}^{a} u_{c}\left(B^{-1} Z\right)^{c}+\frac{\gamma-1}{|u|_{h}^{2}} u^{a}\left(Q_{u, h}\right)_{i c}\left(B^{-1} Z\right)^{c} .
\end{aligned}
$$

(In the final line (A.1) is used to express $\zeta$ as a function of $Z$.) Notice that $\zeta$ is affine in $Z$, and introduce

$$
\zeta_{i J}^{a}=\frac{\partial}{\partial Z^{J}} \zeta_{i}^{a}
$$

The following identities can be verified directly,

$$
\begin{aligned}
& B_{a}^{J} \zeta_{i J}^{a}=\frac{\gamma^{2} u_{i}}{q^{2}} \\
& u_{a} \zeta_{i I}^{a}=\left(B^{-1}\right)_{I}^{b}\left((\gamma-1)\left(Q_{u, h}\right)_{i b}+\frac{\gamma^{2}|u|_{h}^{2}}{q^{2}}\left(P_{u, h}\right)_{i b}\right), \\
& B_{b}^{K} u^{b} \zeta_{i K}^{a}=\frac{\gamma-1}{\gamma}\left(Q_{u, h}\right)_{i}^{a}+\frac{\gamma^{2}|u|_{h}^{2}}{q^{2}}\left(P_{u, h}\right)_{i}^{a}, \\
& \left(B_{a}^{I} \zeta_{i J}^{a}+B_{a}^{J} \zeta_{i I}^{a}\right)=\frac{\gamma}{q^{2}}\left((B u)^{I}\left(\gamma P_{i}^{a}+Q_{i}^{a}\right) B_{a}^{J}+(B u)^{J}\left(\gamma P_{i}^{a}+Q_{i}^{a}\right) B_{a}^{I}\right)
\end{aligned}
$$

\section{A.6. Multipliers}

In this appendix formulae are given for the elements of the generalised null space of $\tilde{\mathcal{M}}^{*}$, the $L^{2}$ adjoint of the operator defined in (2.14), in the case of flat space-time. From these will be derived the inhomogeneous terms in (2.18) for the general case. In the Euclidean case $q=1, h_{i j}=\delta_{i j}$ the formulae for the generalised null space have been given in [23]. As usual they are generated by differentiation with respect to the parameters $\lambda_{A}$ and then taking the simplest possible linear combinations. The definitions made here depend upon an arbitrary spatially homogeneous metric $\underline{h}$ and can be obtained by a change of variables from those in [23].

To start with, the operator in question is given explicitly by:

$$
\tilde{\mathcal{M}}^{*}(a, b)=\left(\partial_{t} a+i \omega q \gamma a-M\left(\lambda, \underline{g}^{\varepsilon}, \underline{h}\right), \partial_{t} b+i \omega q \gamma b+\frac{p^{\varepsilon}}{\sqrt{\mathbf{g}^{\varepsilon}}} a\right) .
$$

Now given $\lambda=(\omega, \theta, \xi, u) \in \mathbb{R}^{2} \times T \Sigma$ and the metric $\underline{h}=-q^{2} d t^{2}+h_{i j} d x^{i} d x^{j}$ define the following exponentially decaying functions of $Z$ (written with the convention that $f_{\omega}, g_{\omega}$ are evaluated with $\operatorname{argument} Z$ ):

$$
\begin{aligned}
& b_{-1}(Z ; \lambda, \underline{h})=g_{\omega}-i \frac{Z \cdot(B u)}{q} f_{\omega} \quad \text { where } g_{\omega}=\partial_{\omega} f_{\omega}, \\
& a_{-1}(Z ; \lambda, \underline{h})=\frac{-i \sqrt{\mathbf{h}}}{\gamma} f_{\omega}+\frac{\sqrt{\mathbf{h}}}{q}\left(\gamma(B u) \cdot \nabla_{Z}-i \omega q \gamma\right) b_{-1},
\end{aligned}
$$




$$
\begin{gathered}
b_{0}(Z ; \lambda, \underline{h})=i f_{\omega}, \\
a_{0}(Z ; \lambda, \underline{h})=\frac{\sqrt{\mathbf{h}}}{q}\left(i \gamma(B u) \cdot \nabla_{Z} f_{\omega}+\omega q \gamma f_{\omega}\right), \\
b_{I}(Z ; \lambda, \underline{h})=\frac{\partial f_{\omega}}{\partial Z^{I}}, \\
a_{I}(Z ; \lambda, \underline{h})=\frac{\sqrt{\mathbf{h}}}{q}\left(\gamma(B u) \cdot \nabla_{Z} \frac{\partial f_{\omega}}{\partial Z^{I}}-i \omega q \gamma \frac{\partial f_{\omega}}{\partial Z^{I}}\right), \\
b_{n+i}(Z ; \lambda, \underline{h})=B_{a}^{I} \zeta_{i}^{a} \frac{\partial f_{\omega}}{\partial Z^{I}}-\frac{i \omega \gamma}{q}\left(\gamma P_{u}+Q_{u}\right)_{i c}\left(B^{-1} Z\right)^{c} f_{\omega}, \\
a_{n+i}(Z ; \lambda, \underline{h})=\frac{\sqrt{\mathbf{h}}}{q}\left(\left(\gamma P_{u, h}+Q_{u, h}\right)_{i}^{j} B_{j}^{I} \frac{\partial f_{\omega}}{\partial Z^{I}}+\left(\gamma(B u) \cdot \nabla_{Z}-i \omega q \gamma\right) b_{n+i}\right) .
\end{gathered}
$$

Here $\gamma$ is as in (1.21).

Remark A.3. - Notice that the dependence on $\lambda=(\omega, \theta, \xi, u)$ is only through $(\omega, u)$. The convention introduced following (M-3) about not explicitly indicating dependence on $B$ as well as $h$ is used here.

\section{LEMMA A.4. - The map}

$$
(\lambda, \underline{h}) \mapsto\left(b_{A}(\cdot ; \lambda, \underline{h}), a_{A}(\cdot ; \lambda, \underline{h})\right) \in H^{k} \times H^{k-1},
$$

defined for $\underline{h}$ in any $\mathrm{Met}^{K}$ and $\lambda \in O^{\underline{h}}$, is continuous for $k=3, C^{1}$ for $k=2$ and $C^{2}$ for $k=1$.

In the case when $\underline{g}^{\varepsilon}=\underline{h}=$ const and $\lambda$ satisfies (1.24) the operator $\tilde{\mathcal{M}}^{*}$ reduces, on functions of $Z$ (where $Z$ is as in (1.22)), to the operator

$$
\tilde{\mathcal{M}}_{0}^{*}(a, b)=\left(-\gamma(B u) \cdot \nabla_{Z} a+i \omega q \gamma a-M(\lambda, \underline{h}, \underline{h}) b,-\gamma(B u) \cdot \nabla_{Z} b+i \omega q \gamma b+\frac{q}{\sqrt{\mathbf{h}}} a\right) .
$$

Calculation, using (A.19)-(A.22), then shows that the pairs of functions $\left(a_{A}, b_{A}\right)$ lie in the generalised null space: $\tilde{\mathcal{M}}_{0}^{*}\left(a_{A}, b_{A}\right)=0$ if $A=0$ or if $A=I \in\{1, \ldots, n\}$ while

$$
\tilde{\mathcal{M}}_{0}^{*}\left(a_{-1}, b_{-1}\right)=-\frac{q}{\gamma}\left(a_{0}, b_{0}\right) \quad(A=-1)
$$

and

$$
\tilde{\mathcal{M}}_{0}^{*}\left(a_{n+i}, b_{n+i}\right)=\frac{\sqrt{\mathbf{h}}}{q}(\gamma P+Q)_{i}^{j} B_{j}^{I}\left(a_{I}, b_{I}\right) \quad(A=n+i) .
$$

Now in the general case when $\lambda$ does not satisfy (1.24), when $\underline{h}$ is time-dependent and $\underline{g}^{\varepsilon}$ is both space and time dependent the following formula holds

$$
\begin{aligned}
\tilde{\mathcal{M}}^{*}(a, b)= & \tilde{\mathcal{M}}_{0}^{*}(a, b)+\varepsilon \mu^{I} \frac{\partial}{\partial Z^{I}}(a, b)+\varepsilon\left(\dot{\lambda} D_{\lambda}+\underline{\dot{h}} D_{\underline{h}}\right)(a, b) \\
& +\left(\left(M(\lambda, \underline{h}, \underline{h})-M\left(\lambda, \underline{g}^{\varepsilon}, \underline{h}\right)\right) b,\left(\frac{p^{\varepsilon}}{\sqrt{\mathbf{g}^{\varepsilon}}}-\frac{q}{\sqrt{\mathbf{h}}}\right) a\right)
\end{aligned}
$$


where $\mu^{I}$ is as in (A.8). From this follows (2.18), where the only non-zero elements of the matrix $D_{A B}$ are $D_{-1,0}=-q / \gamma$ and

$$
D_{n+i, I}=\frac{\sqrt{\mathbf{h}}}{q}(\gamma P+Q)_{i}^{j} B_{j}^{I}
$$

and the inhomogeneous terms are given by

$$
\begin{aligned}
\varepsilon\left(\mathbf{J}_{A}^{1}, \mathbf{J}_{A}^{2}\right)= & \varepsilon \mu^{I} \frac{\partial}{\partial Z^{I}}\left(a_{A}, b_{A}\right)+\varepsilon\left(\dot{\Lambda} D_{\lambda}+\underline{\dot{h}} D_{\underline{h}}\right)\left(a_{A}, b_{A}\right) \\
& +\left(\left(M(\lambda, \underline{h}, \underline{h})-M\left(\lambda, \underline{g}^{\varepsilon}, \underline{h}\right)\right) b_{A},\left(\frac{p^{\varepsilon}}{\sqrt{\mathbf{g}^{\varepsilon}}}-\frac{q}{\sqrt{\mathbf{h}}}\right) a_{A}\right) .
\end{aligned}
$$

Remark A.5. - The $\left(a_{A}, b_{A}\right)$ are functions of $(Z, \lambda, \underline{h})$ but the explicit dependence on $\lambda=(\omega, \theta, \xi, u)$ is only through $(\omega, u)$ which is why it is possible to replace $\dot{\lambda}$ with $\dot{\Lambda}$ here.

The inhomogeneous terms are exponentially decaying functions of $Z$ which also have the following parametric dependence:

$$
\mathbf{J}_{A}^{1,2}=\mathbf{J}_{A}^{1,2}\left(Z ; \lambda, \dot{\Lambda}, \underline{h}, \underline{\dot{h}}, \underline{g}^{\varepsilon}, \underline{g}^{\varepsilon}, \nabla \underline{g}^{\varepsilon}\right) .
$$

They have the following form (using the summation convention):

$$
\mathbf{J}_{A}^{1,2}=\underline{\mathbf{J}}_{A}^{1,2}+\underline{\mathbf{J}}_{A B}^{1,2} \dot{\Lambda}^{B}+\underline{\mathbf{J}}_{\mu \nu}^{1,2} \underline{\underline{h}}_{\mu \nu}+\underline{\mathbf{J}}_{\alpha}^{1,2} Q_{\alpha}\left(\underline{g}^{\varepsilon}, D \underline{g}^{\varepsilon}\right)
$$

where the $Q_{\alpha}$ are affine in their second argument and rational in their first argument and all of the $\underline{\mathbf{J}}_{\{\cdot\}}^{1,2}$ have dependence $\underline{\mathbf{J}}_{\{\cdot\}}^{1,2}=\underline{\mathbf{J}}_{\{\cdot\}}^{1,2}(Z ; \lambda, \underline{h})$.

LEMMA A.6. - The maps

$$
(\lambda, \underline{h}) \mapsto\left(\underline{\mathbf{J}}_{\{\cdot\}}^{2}(\cdot ; \lambda, \underline{h}), \underline{\mathbf{J}}_{\{\cdot\}}^{1}(\cdot ; \lambda, \underline{h})\right) \in H^{k} \times H^{k-1},
$$

defined for $\underline{h}$ in any $\mathrm{Met}^{K}$ and $\lambda \in O^{\underline{h}}$, are continuous for $k=2$ and $C^{1}$ for $k=1$. Furthermore $\varepsilon\left\langle\mathbf{J}_{A}^{2}, w\right\rangle=\varepsilon \tilde{F}_{A}$ with

$$
\tilde{F}_{A}=\tilde{F}_{0 A}\left(t, \lambda ; w, \underline{g}^{\varepsilon}, \underline{h}, \varepsilon\right)+\tilde{F}_{A B}\left(t, \lambda ; w, \underline{g}^{\varepsilon}, \underline{h}, \varepsilon\right) \dot{\Lambda}_{B}+\tilde{F}_{\mu \nu}\left(t, \lambda ; w, \underline{g}^{\varepsilon}, \underline{h}, \varepsilon\right) \underline{\hat{h}}_{\mu \nu}
$$

where the $\tilde{F}_{\{\cdot\}}$ are continuous functions of $t, \lambda$ and of $w \in H^{-1}$ and are $\leqslant c\|\varepsilon w\|_{H^{-1}}$ with $c=c\left(K_{1}, \bar{\gamma}\left(q^{-1}|u|_{h}\right),\left(m^{2}-\omega^{2}\right)^{-1}\right)$. Similarly $\varepsilon\left\langle\mathbf{J}_{A}^{1}, v\right\rangle=\varepsilon \tilde{G}_{A}$ with

$$
\tilde{G}_{A}=\tilde{G}_{0 A}\left(t, \lambda ; v, \underline{g}^{\varepsilon}, \underline{h}, \varepsilon\right)+\tilde{G}_{A B}\left(t, \lambda ; v, \underline{g}^{\varepsilon}, \underline{h}, \varepsilon\right) \dot{\Lambda}_{B}+\tilde{G}_{\mu \nu}\left(t, \lambda ; v, \underline{g}^{\varepsilon}, \underline{h}, \varepsilon\right) \underline{\dot{h}}_{\mu \nu}
$$

where the $\tilde{G}_{\{\cdot\}}$ are continuous functions of $t, \lambda$ and of $v \in L^{2}$ and are $\leqslant c\|\varepsilon v\|_{L^{2}}$ with $c=c\left(K_{1}, \bar{\gamma}\left(q^{-1}|u|_{h}\right),\left(m^{2}-\omega^{2}\right)^{-1}\right)$.

Proof. - This lemma can be essentially read off from (A.29) using Lemmas A.4 and A.10. The stated estimates are given in the form needed in Section 2.2, and are not necessarily optimal.

\section{A.7. Inhomogeneous terms}

In this appendix formulae and estimates for the inhomogeneous terms in (2.12) are given. Thus it is assumed that 
- $t \mapsto \lambda(t)=(\omega(t), \theta(t), \xi(t), u(t))$ is a $C^{1}$ function and $\Lambda(t)$ is defined as in (2.7), with time evolution as in (2.30), (2.32),

- $t \mapsto\left(\phi(t, \psi(t))\right.$ is a solution of (NLW $\left.\underline{\tilde{g}}^{\varepsilon}\right)$, with regularity as in (WP-1)-(WP-2),

and the various inhomogeneous terms $j_{i}, \tilde{j}_{i}, f_{i}, \tilde{f}_{i}, \mathcal{N}, \tilde{\mathcal{N}}$ are as defined in (2.12) and (2.41)(2.42) and the surrounding discussion. The explicit formulae are:

$$
\begin{aligned}
j_{1}(t, x ; \lambda, \dot{\Lambda}, \underline{h}, \underline{\dot{h}})= & -e^{-i \Theta}\left\langle D_{\lambda} \phi_{S}, \dot{\Lambda}\right\rangle-e^{-i \Theta}\left\langle D_{\underline{h}} \phi_{S}, \underline{\dot{h}}\right\rangle \\
= & -\dot{\omega} g_{\omega}-\mu^{I} \frac{\partial f_{\omega}}{\partial Z^{I}}-i \mu_{0} f_{\omega}, \quad \text { where } g_{\omega}=\partial_{\omega} f_{\omega} \\
j_{2}(t, x ; \lambda, \dot{\Lambda}, \underline{h}, \underline{\dot{h}})= & -e^{-i \Theta}\left\langle D_{\lambda} \psi_{S}, \dot{\Lambda}\right\rangle-e^{-i \Theta}\left\langle D_{\underline{h}} \psi_{S}, \underline{\dot{h}}\right\rangle \\
= & \partial_{T}\left(\frac{\gamma \sqrt{\mathbf{h}}}{q} B u\right) \cdot \nabla_{Z} f_{\omega}-i \partial_{T}(\omega \gamma \sqrt{\mathbf{h}}) f_{\omega} \\
& -\partial_{T} \omega\left(i \omega \gamma \sqrt{\mathbf{h}} g_{\omega}-\frac{\gamma \sqrt{\mathbf{h}}}{q}(B u) \cdot \nabla_{Z} g_{\omega}\right) \\
& -\mu^{I}\left(i \omega \gamma \sqrt{\mathbf{h}}-\frac{\gamma \sqrt{\mathbf{h}}}{q}(B u) \cdot \nabla_{Z}\right) \frac{\partial f_{\omega}}{\partial Z^{I}} \\
& -i \mu^{0}\left(i \omega \gamma \sqrt{\mathbf{h}}-\frac{\gamma \sqrt{\mathbf{h}}}{q}(B u) \cdot \nabla_{Z}\right) f_{\omega}
\end{aligned}
$$

$$
\begin{aligned}
f_{1}\left(t, x ; \lambda, \underline{g}^{\varepsilon}, \underline{h}\right)= & \frac{1}{\varepsilon}\left(\frac{p^{\varepsilon}}{\sqrt{\mathbf{g}}}-\frac{q}{\sqrt{\mathbf{h}}}\right) e^{-i \Theta} \psi_{S}, \\
f_{2}\left(t, x ; \lambda, \underline{g}^{\varepsilon}, \underline{h}\right)= & \frac{e^{-i \Theta}}{\varepsilon}\left(\Delta_{p^{\varepsilon}, g^{\varepsilon}}-\Delta_{q, h}\right)\left(\phi_{S}\right) \\
& -\frac{e^{-i \Theta}}{\varepsilon}\left(p^{\varepsilon} \sqrt{\mathbf{g}^{\varepsilon}}-q \sqrt{\mathbf{h}}\right)\left(m^{2} \phi_{S}-\beta\left(\left|\phi_{S}\right|\right) \phi_{S}\right), \\
\mathcal{N}\left(f_{\omega}, \varepsilon v, \underline{g}^{\varepsilon}, \varepsilon\right)= & \frac{1}{\varepsilon^{2}} p^{\varepsilon} \sqrt{\mathbf{g}^{\varepsilon}}\left\{\beta\left(\left|f_{\omega}+\varepsilon v\right|\right)\left(f_{\omega}+\varepsilon v\right)-\beta\left(f_{\omega}\right) f_{\omega}\right. \\
& \left.-\varepsilon \beta\left(f_{\omega}\right) v-\varepsilon f_{\omega} \beta^{\prime}\left(f_{\omega}\right) \Re v\right\} .
\end{aligned}
$$

In these formulae $\phi_{S}, \psi_{S}, Z, \Theta$ are as in Section 2.1. Notice that $j_{1}, j_{2}$ are linear in $\dot{\Lambda}, \underline{\dot{h}}$, or, after substituting for $\underline{\dot{h}}$ from (2.45) affine in $\dot{\Lambda}$. The regularity properties of these terms as functions of $(\lambda, \underline{h})$ can be read off from hypothesis $(\mathrm{R})$ :

Lemma A.7. - The map $(\lambda, \underline{h}) \mapsto j_{1}$ (resp. $j_{2}$ ) are continuous to $H^{3}$ (resp. $H^{2}$ ), $C^{1}$ to $H^{2}$ (resp. $\left.H^{1}\right)$, and $C^{2}$ to $H^{1}\left(\right.$ resp. $\left.L^{2}\right)$, for $\underline{h}$ in any $\operatorname{Met}^{K}$ and $\lambda \in O^{\underline{h}}$.

Lemma A.8. - There exists $c=c\left(K_{0},\left(m^{2}-\omega^{2}\right)^{-1}, \bar{\gamma}\left(|u|_{h} / q\right), \varepsilon\|v\|_{H^{1}}\right)$, where $K_{0}$ is as in (M-1)-(M-3), such that

$$
\left\|\varepsilon \mathcal{N}\left(f_{\omega}, \varepsilon v, \underline{g}^{\varepsilon}, \varepsilon\right)\right\|_{L^{2}} \leqslant c \varepsilon\|v\|_{H^{1}}^{2} .
$$

Proof. - It is convenient to use the variable $\tilde{\phi}=e^{i \Theta} v$ and $\mathcal{F}$ instead of $\beta$ (see (1.1)). Now compute, suppressing arguments where possible,

$$
\begin{aligned}
\|\mathcal{N}\|_{L^{2}}=\left\|e^{i \Theta} \mathcal{N}\right\|_{L^{2}} & =\varepsilon^{-2}\left\|p^{\varepsilon} \sqrt{\mathbf{g}^{\varepsilon}}\left(\mathcal{F}\left(\phi_{S}+\varepsilon \tilde{\phi}\right)-\mathcal{F} l\left(\phi_{S}\right)\right)-\varepsilon\left\langle\mathcal{F}^{\prime}\left(\phi_{S}\right), \tilde{\phi}\right\rangle\right\|_{L^{2}} \\
& =\varepsilon^{-1}\left\|p^{\varepsilon} \sqrt{\mathbf{g}^{\varepsilon}} \int_{0}^{1}\left\langle\mathcal{F}^{\prime}\left(\phi_{S}+\varepsilon \tau \tilde{\phi}\right)-\mathcal{F}^{\prime}\left(\phi_{S}\right), \tilde{\phi}\right\rangle d \tau\right\|_{L^{2}}
\end{aligned}
$$


Recalling now the hypotheses (NL-3) the result follows by applying Holder's inequality with $1 / 2=1 / 2^{*}+1 / n$.

The estimates involving the operators

$$
\begin{aligned}
\boldsymbol{X} & \equiv \partial_{t}+\tilde{U}^{i}\left(\nabla_{x}\right)_{i}, \\
\tilde{\boldsymbol{X}} & \equiv \partial_{t}-\frac{i \omega p^{\varepsilon}}{\gamma}+\tilde{U}^{i}\left(\nabla_{x}\right)_{i}
\end{aligned}
$$

needed in Section 2.3 are now given. Recall the decomposition in (2.46)-(2.47).

Lemma A.9. - The inhomogeneous terms $\tilde{j}_{i}^{\bullet}$ appearing in (2.46)-(2.47) are of the form

$$
\begin{aligned}
& \tilde{j}_{1}^{(0)}(t, x)=-\left\langle D_{\lambda} \phi_{S}, \mathbf{V}_{1}\right\rangle-\left\langle D_{\underline{h}} \phi_{S}, \underline{\dot{h}}^{(0)}\right\rangle, \\
& \tilde{j}_{2}^{(0)}(t, x)=-\left\langle D_{\lambda} \psi_{S}, \mathbf{V}_{1}\right\rangle-\left\langle D_{\underline{h}} \psi_{S}, \underline{\dot{h}}^{(0)}\right\rangle, \\
& \tilde{j}_{1}^{(1)}(t, x)=-\left\langle D_{\lambda} \phi_{S}, \mathbf{V}_{2}\right\rangle-\left\langle D_{\underline{h}} \phi_{S}, \underline{\dot{h}}^{(1)}\right\rangle, \\
& \tilde{j}_{2}^{(1)}(t, x)=-\left\langle D_{\lambda} \psi_{S}, \mathbf{V}_{2}\right\rangle-\left\langle D_{\underline{h}} \psi_{S}, \underline{\dot{h}}^{(1)}\right\rangle
\end{aligned}
$$

and satisfy the estimates

$$
\begin{aligned}
& \left\|\tilde{\boldsymbol{X}} \tilde{j}_{i}^{(0)}\right\|_{L^{2}} \leqslant c_{2} \varepsilon\left(1+|\dot{\Lambda}|+\left|\partial_{t} \lambda\right|\right), \\
& \left\|\tilde{\boldsymbol{X}} \tilde{f}_{i}\right\|_{L^{2}} \leqslant c_{1} \varepsilon\left(1+\left|\partial_{t} \lambda\right|+|\dot{\Lambda}|\right), \\
& \left\|\nabla_{x} j_{1}^{(1)}\right\|_{L^{2}}+\left\|j_{i}^{(1)}\right\|_{L^{2}} \leqslant c_{3} \varepsilon
\end{aligned}
$$

with $c_{i}=c_{i}\left(K_{i}, \bar{\gamma}\left(|u|_{h} / q\right),\left(m^{2}-\omega^{2}\right)^{-1}\right)$ for $i=1,2$ and

$$
c_{3}=c_{3}\left(K_{2}, \bar{\gamma}\left(|u|_{h} / q\right), \Omega, \omega_{0},\|(v, w)\|_{H^{1} \times L^{2}}\right) .
$$

Proof. - To prove the first observe that $\mathbf{V}_{\mathbf{1}}$ is itself a $C^{1}$ function of $\varepsilon t, \lambda$ (as can be seen by inspection of the formulae in Section 2.2) which satisfies (1.52), while (2.45) implies immediately

$$
\left|\underline{\dot{h}}^{(0)}\right|+\left|\partial_{t} \underline{\dot{h}}^{(0)}\right| \leqslant c\left(K_{2}\right)\left(1+\left|\partial_{t} \lambda\right|+|\dot{\Lambda}|\right) .
$$

The result now follows the explicit expressions for the inhomogeneous terms above.

The second follows from the product rule and Lemma A.13. Finally recall from (2.45) that $\underline{\dot{h}}^{(1)}=\varepsilon\left\langle\mathbf{V}_{2}^{i},\left(D_{i} \underline{g}\right)^{\varepsilon}\right\rangle$ so that $\left|\underline{\dot{h}}^{(1)}\right| \leqslant K_{1}\left|\varepsilon \mathbf{V}_{2}\right|$ and the last estimate follows from (A.41)-(A.42) and (2.31).

\section{A.8. Estimates for some integrals}

In this appendix various estimates for integrals used primarily in Sections 2.2 and 2.3 are given.

LEMmA A.10. - (a) Let $F \in C^{1}\left(\mathbb{R}^{n} ; \mathbb{R}\right)$ have bounded first derivative and $\rho$ be an integrable function satisfying

$$
\int(1+|Z|)|\rho(Z)| d Z<\infty
$$


and let $A$ be an $n \times n$ matrix. Then for all $p \geqslant 1$

$$
\left\|(1+|Z|)^{m}(F(\varepsilon(\xi+A Z))-F(\varepsilon \xi)) \rho(Z)\right\|_{L_{d Z}^{p}} \leqslant c \varepsilon|A|\|D F\|_{L^{\infty}}\left\|(1+|Z|)^{m+1} \rho(Z)\right\|_{L_{d Z}^{p}} .
$$

(b) Let $F \in C^{2}\left(\mathbb{R}^{n} ; \mathbb{R}\right)$ have bounded second derivative, A be as above, and $\rho$ be an integrable function satisfying

$$
\int Z \rho(Z) d Z=0 \quad \text { and } \quad \int\left(1+|Z|^{2}\right) \rho(Z) d Z<\infty .
$$

Then

$$
\left|\int(F(\varepsilon(\xi+A Z))-F(\varepsilon \xi)) \rho(Z) d Z\right| \leqslant c \varepsilon^{2}|A|^{2}\left\|D^{2} F\right\|_{L^{\infty}}\left|\left(1+|Z|^{2}\right) \rho(Z)\right|_{L_{d Z}^{1}} .
$$

If further $F \in C^{3}\left(\mathbb{R}^{n} ; \mathbb{R}\right)$ has bounded third derivatives then

$$
\left|\int[D F(\varepsilon(\xi+A Z))-D F(\varepsilon \xi)] \rho(Z) d Z\right| \leqslant c \varepsilon^{2}|A|\left\|D^{3} F\right\|_{L^{\infty}}\left|\left(1+|Z|^{2}\right) \rho(Z)\right|_{L_{d Z}^{1}} .
$$

Proof. - The proof of, for example, the middle inequality follows from the assumptions by writing:

$$
F(\varepsilon(\xi+A Z))-F(\varepsilon \xi)-\varepsilon\langle D F(\varepsilon \xi), A Z\rangle=\int_{0}^{1} \int_{0}^{1} s\left\langle D^{2} F(\varepsilon \xi+s t \varepsilon A Z), \varepsilon A Z, \varepsilon A Z\right\rangle d s d t
$$

The others are proved similarly.

In the following it is assumed, as usual, that $g$ is a pseudo-Riemannian metric of the form (1.44), where $(p, g)$ satisfy the conditions in Section 1.2; it is rescaled as in (1.44) and evaluated at $x=\xi(t)$ as in (1.45) to define a spatially homogeneous metric $\underline{h}=(q, h)$.

LEMmA A.11. - Given $\lambda=(\omega, \theta, \xi, u) \in \mathbb{R}^{2} \times T \Sigma$ consider the affine transformation $Z \mapsto x(Z)$ given by (A.1), which is itself the inverse of the affine transformation defined in (2.4). Assume further that $j$ is an integrable function with $\|(1+|Z|) j(Z)\|_{L_{d Z}^{2}}<\infty$, and given $u \in T_{\xi} \Sigma$ define a vector field $\tilde{U}$ on $\Sigma$ as in (2.48). Then:

$$
\begin{aligned}
& \left\|(1+|Z|)^{m}\left(p^{\varepsilon}(x(Z))-q\right) j(Z)\right\|_{L^{2}} \leqslant \varepsilon c\left\|(1+|Z|)^{m+1} j(Z)\right\|_{L^{2}}, \\
& \left\|(1+|Z|)^{m}\left(\tilde{U}^{i}(x(Z))-u^{i}\right) j(Z)\right\|_{L^{2}} \leqslant \varepsilon c\left\|(1+|Z|)^{m+1} j(Z)\right\|_{L^{2}}
\end{aligned}
$$

with $c=c\left(K_{1}, \bar{\gamma}\left(q^{-1}|u|_{h}\right)\right)$.

Proof. - By (A.1) $x(Z)=\xi+A Z$ where $A$ is a linear transformation with norm bounded (in terms of $K_{i}$ for any $\left.i \geqslant 0\right)$ and $\bar{\gamma}\left(q^{-1}|u|_{h}\right)$. Now estimate as follows

$$
\int|(p(\varepsilon \xi+\varepsilon A Z)-p(\varepsilon \xi)) j(Z)|^{2} d Z \leqslant c\|(1+|Z|) j(Z)\|_{L_{d Z}^{2}}^{2}
$$

since the derivatives of $p$ are bounded by $K_{1}$. The second inequality is proved similarly. 
LEMMA A.12. - Given a $C^{1}$ curve

$$
t \mapsto \lambda(t)=(\omega, \theta, \xi, u) \in O^{g^{\varepsilon}}, t
$$

let the functions $Z=Z(t, x)$ and $\Theta=\Theta(t, x)$ be as in (2.4), introduce the first order differential operators $\boldsymbol{X}, \tilde{\boldsymbol{X}}$ as in (A.37)-(A.38), and define $\mathbf{V}_{*}$ by

$$
\varepsilon \mathbf{V}_{*}(t)=\partial_{t} \lambda(t)-\mathbf{V}_{\mathbf{0}}(\lambda(t) ; \underline{h}(t)) .
$$

Assuming that $\|(1+|Z|) j(Z)\|_{L_{d Z}^{2}}^{2}+\left\|(1+|Z|) \nabla_{Z} j(Z)\right\|_{L_{d Z}^{2}}^{2}<\infty$ and that the function $\tilde{j}(t, x)$ is of the form $\tilde{j}=e^{i \Theta} j(Z)$ the following estimates hold:

$$
\begin{aligned}
& \left\|(1+|Z|)^{m} \boldsymbol{X} j\right\|_{L^{2}} \leqslant c_{1} \varepsilon\left(1+\left|\partial_{t} \lambda\right|+\left|\mathbf{V}_{*}\right|\right)\left\|(1+|Z|)^{m+1}\left(|j(Z)|+\left|\nabla_{Z} j(Z)\right|\right)\right\|_{L^{2}}, \\
& \left\|(1+|Z|)^{m} \tilde{\boldsymbol{X}} \tilde{j}\right\|_{L^{2}} \leqslant c_{1} \varepsilon\left(1+\left|\partial_{t} \lambda\right|+\left|\mathbf{V}_{*}\right|\right) \times\left\|(1+|Z|)^{m+1}\left(|j(Z)|+\left|\nabla_{Z} j(Z)\right|\right)\right\|_{L^{2}}, \\
& \left\|(1+|Z|)^{m} \nabla_{x}(\boldsymbol{X} j)\right\|_{L^{2}} \leqslant c_{2} \varepsilon\left(1+\left|\partial_{t} \lambda\right|+\left|\mathbf{V}_{*}\right|\right)\left\|(1+|Z|)^{m+1}\left(|j(Z)|+\left|\nabla_{Z}^{2} j(Z)\right|\right)\right\|_{L^{2}}, \\
& \left\|(1+|Z|)^{m} \nabla_{x}(\tilde{\boldsymbol{X}} \tilde{j})\right\|_{L^{2}} \leqslant c_{2} \varepsilon\left(1+\left|\partial_{t} \lambda\right|+\left|\mathbf{V}_{*}\right|\right)\left\|(1+|Z|)^{m+1}\left(|j(Z)|+\left|\nabla_{Z}^{2} j(Z)\right|\right)\right\|_{L^{2}}, \\
& \text { with } c_{i}=c_{i}\left(K_{i}, \bar{\gamma}\left(q^{-1}|u|_{h}\right) \text { for } i=1,2 .\right.
\end{aligned}
$$

Proof. - This follows directly by using the identities

$$
\begin{aligned}
& \partial_{t} \Theta+\tilde{U}^{i} \partial_{x^{i}} \Theta-\frac{\omega p^{\varepsilon}}{\gamma}=\varepsilon \mu^{0}+\frac{\omega}{\gamma}\left(q-p^{\varepsilon}\right)-\frac{\omega \gamma}{q}\left(\tilde{U}^{i}-u^{i}\right) u_{i}, \\
& \partial_{t} Z^{I}+\tilde{U}^{i} \partial_{x^{i}} Z^{I}=\varepsilon \mu^{I}+\left(\tilde{U}^{i}-u^{i}\right) B_{j}^{I}\left(\gamma P_{u, h}+Q_{u, h}\right)_{i}^{j},
\end{aligned}
$$

where, for all $A, \mu^{A}(t, x)=\boldsymbol{\mu}^{A}\left(x, \lambda(t), \mathbf{V}_{*}(t), \underline{h}(t), \underline{\underline{h}}(t)\right)$ is as in (A.10) and $\underline{\dot{h}}$ is as in (2.45), to reduce to Lemma A.11.

LEMMA A.13. - In the situation of Lemma A.12 assume in addition that there is given a function $\alpha \in C^{2}\left(\mathbb{R}^{1+n}, \mathbb{R}\right)$ and $a(t) \equiv \alpha(\varepsilon t, \varepsilon \xi(t))$. Then the following estimates hold for $\varepsilon$ small:

$$
\begin{aligned}
& \left\|(1+|Z|)^{m} \boldsymbol{X}\left(\varepsilon^{-1}(\alpha(\varepsilon t, \varepsilon x)-a(t)) j(Z(t, x))\right)\right\|_{L^{2}} \\
& \quad \leqslant \varepsilon c \times\left(1+\left|\partial_{t} \lambda\right|+\left|\mathbf{V}_{*}\right|\right) \times\left(\left\|(1+|Z|)^{m+2}\left(|j(Z)|+\left|\nabla_{Z} j(Z)\right|\right)\right\|_{L^{2}}\right) \\
& \left\|(1+|Z|)^{m} \nabla_{x}\left[\left(\boldsymbol{X} \varepsilon^{-1}(\alpha(\varepsilon t, \varepsilon x)-a(t)) j(Z(t, x))\right)\right]\right\|_{L^{2}} \\
& \quad \leqslant \varepsilon c \times\left(1+\left|\partial_{t} \lambda\right|+\left|\mathbf{V}_{*}\right|\right) \times\left(\left\|(1+|Z|)^{m+2}\left(|j|+\left|\nabla_{Z} j\right|+\left|\nabla_{Z}^{2} j\right|\right)\right\|_{L^{2}}\right)
\end{aligned}
$$

with $c=c\left(K_{1}, \bar{\gamma}\left(q^{-1}|u|_{h},|\alpha|_{C^{2}}\right)\right.$, as do the analogous statements with $j$ replaced by $\tilde{j}$ and $\tilde{\boldsymbol{X}}$ replacing $\boldsymbol{X}$.

Remark. - In applications an estimate for $V_{*}$ is provided by Lemma 2.3.

Proof. - Compute

$$
\begin{aligned}
\left(\partial_{t}+\right. & \left.\tilde{U}^{i}(t) \nabla_{i}\right) \varepsilon^{-1}(\alpha(\varepsilon t, \varepsilon x)-a(t)) j(Z(t, x)) \\
= & {\left[\partial_{1} \alpha(\varepsilon t, \varepsilon x)-\partial_{1} \alpha(\varepsilon t, \varepsilon \xi(t))\right.} \\
& -\left(\partial_{t} \xi-u\right) \partial_{2} \alpha(\varepsilon t, \varepsilon \xi)-(u-\tilde{U}) \partial_{2} \alpha(\varepsilon t, \varepsilon \xi) \\
& \left.+\tilde{U}\left(\partial_{2} \alpha(\varepsilon t, \varepsilon x)-\partial_{2} \alpha(\varepsilon t, \varepsilon \xi(t))\right)\right] j(Z(t, x)) \\
& +\varepsilon^{-1}(\alpha(\varepsilon t, \varepsilon x)-a(t))\left(\partial_{t}+\tilde{U}^{i}(t) \nabla_{i}\right) j(Z(t, x)) .
\end{aligned}
$$


Use Lemma A.10 to estimate the first third and fourth lines and (A.43) and Lemma A.11 for the second. This gives the first estimate. The second is proved similarly, observing in addition that a spatial derivative applied to $\partial \alpha(\varepsilon t, \varepsilon x)$ introduces an additional factor of $\varepsilon$.

\section{A.9. Computation of the modulation equations (proof of Lemma 2.2)}

In this appendix the $L^{2}$ inner products which appear in (2.19) are computed in order to obtain the modulation equations (2.24)-(2.27) and prove Lemma 2.2. Assume $\lambda=\lambda(t)$ lies in a compact subset of the parameter space (1.19). Since time $t$ is fixed (and will be suppressed) in this section, it is possible to regard $Z$, defined in (2.4), as the independent variable rather than $x$. Thus all functions of $x$ will be regarded as functions of $Z$ (obtained by composition with the inverse function $x \mapsto \mathbf{x}(Z ; \lambda, \underline{h})$ given in (A.1)), but by abuse of notation will be represented by the same symbols. Since, as explained in Appendix A.1, the measures $d Z=d^{n} Z$ and $d x=d^{n} x$ differ only by a nowhere vanishing function of time, it is permissible in condition (2.19) to replace $d x$ by $d Z$. Also it will be convenient to suppress the subscripts $u, h$ on the projection operators $P, Q$ and to write $\gamma$ for $\bar{\gamma}\left(|u|_{h} / q\right)$. The notation $\langle$,$\rangle and \|\cdot\|$ will be used for the $L_{d Z}^{2}$ inner product and norm respectively.

It will transpire that the terms on the left hand side of (2.24)-(2.27) arise from the inner products involving $j_{1}, j_{2}, f_{1}, f_{2}$, up to $\mathrm{O}(\varepsilon)$ terms which are continuous in $t, \lambda$ and lie in the class $\mathfrak{E}_{1}$ (see (2.20)) and hence contribute to the right hand sides. (Continuity follows immediately from the assumption (R) in Section 1.7). The other contributions to the right hand side come from the terms with an $\varepsilon$ explicitly in front; these will be discussed first.

Term involving $\mathcal{N}$. The term involving $\mathcal{N}$ is independent of $\dot{\Lambda}$, and, by Lemma A.8, satisfies the bound (for any $\varepsilon<1$ ):

$$
\left|\left\langle b_{A}(Z ; \lambda, \underline{h}), \varepsilon \mathcal{N}\right\rangle\right| \leqslant \varepsilon c .\|v\|_{H^{1}}^{2}
$$

where $\left.\left.c=c\left(q^{-1}|u|_{h}\right),\left(m^{2}-\omega^{2}\right)^{-1}\right), \varepsilon\|v\|_{H^{1}}, K_{0}, \tilde{K}_{2}\right)$. Now $\left\langle b_{A}(Z ; \lambda, \underline{h}), \varepsilon \mathcal{N}\right\rangle$ is a continuous function of $\lambda$, and also of $v$ (in the strong $H^{1}$ topology) since the nonlinearity is not allowed to have supercritical growth by (NL-1)-(NL-3). Therefore this term lies in the class $\mathfrak{E}_{2}$ (see (2.21)) and contributes to the term $\tilde{F}_{A}^{(0)}$, and is bounded as in (2.21) (with $c$ depending also on $K_{0}$ and the $\tilde{K}_{i}$ of (NL-1)-(NL-3).)

Terms involving $(v, w)$ explicitly. The other terms with an $\varepsilon$ appearing in front are those in the second line of (2.19). These are treated directly using Lemma A. 6 and substituting for $\underline{\dot{h}}$ from (1.45). It follows that these terms can be broken up into two terms which contribute, respectively, to $\tilde{F}_{A}^{(0)}$ and $\tilde{F}_{A B}$ in (2.24)-(2.27) and as functions of $t, \lambda$ are bounded as in (2.21)-(2.23) with constants $c$ (which depend also on $K_{1}$ ).

Terms involving $j_{1}, j_{2}$. The contributions from $j_{1}, j_{2}$ may be computed directly:

$$
\begin{aligned}
\left\langle b_{-1}, j_{2}\right\rangle+\left\langle a_{-1}, j_{1}\right\rangle= & \gamma^{-1} \sqrt{\mathbf{h}} \tilde{\mu}^{0}\left\|f_{\omega}\right\|^{2}-\frac{\gamma \sqrt{\mathbf{h}}}{q}(B u)^{J} \tilde{\mu}^{J}\left(\frac{2}{n}\left\langle\nabla g_{\omega}, \nabla f_{\omega}\right\rangle+\omega\left\|f_{\omega}\right\|^{2}\right) \\
& +\gamma \sqrt{\mathbf{h}} \tilde{\mu}^{0}\left(2 \omega\left\langle f_{\omega}, g_{\omega}\right\rangle+\frac{|u|_{h}^{2}}{q^{2}}\left\|f_{\omega}\right\|^{2}\right) \\
= & \sqrt{\mathbf{h}}\left(\gamma^{-1}+\gamma \frac{|u|_{h}^{2}}{q^{2}}\right)\left\|f_{\omega}\right\|^{2} \tilde{\mu}^{0}+2 \omega \gamma \sqrt{\mathbf{h}} \tilde{\mu}^{0}\left\langle f_{\omega}, g_{\omega}\right\rangle \\
= & \gamma \sqrt{\mathbf{h}} \tilde{\mu}^{0} \frac{\partial}{\partial \omega}\left(\omega\left\|f_{\omega}\right\|^{2}\right),
\end{aligned}
$$




$$
\begin{aligned}
\left\langle b_{0}, j_{2}\right\rangle+\left\langle a_{0}, j_{1}\right\rangle & =-\partial_{T}(\omega \gamma \sqrt{\mathbf{h}})\left\|f_{\omega}\right\|^{2}-2 \omega \gamma \sqrt{\mathbf{h}} \partial_{T} \omega\left\langle f_{\omega}, g_{\omega}\right\rangle+\omega \gamma \sqrt{\mathbf{h}}\left\|f_{\omega}\right\|^{2} \frac{\partial \mu^{I}}{\partial Z^{I}} \\
& =-\partial_{T}\left(\omega \gamma \sqrt{\mathbf{h}}\left\|f_{\omega}\right\|^{2}\right)+\omega \gamma\left\|f_{\omega}\right\|^{2} \frac{\partial \mu^{I}}{\partial Z^{I}} \\
& =-\gamma \sqrt{\mathbf{h}} \partial_{T}\left(\omega\left\|f_{\omega}\right\|^{2}\right) .
\end{aligned}
$$

For $I \in\{1, \ldots, n\}$

$$
\begin{aligned}
\left\langle b_{I}, j_{2}\right\rangle+\left\langle a_{I}, j_{1}\right\rangle= & \frac{1}{n}\left\|\nabla f_{\omega}\right\|^{2} \partial_{T}\left(\frac{\gamma \sqrt{\mathbf{h}}}{q}(B u)^{I}\right)+2 \dot{\omega}\left(\frac{\gamma \sqrt{\mathbf{h}}}{q}(B u)^{J}\right)\left\langle\frac{\partial g_{\omega}}{\partial Z^{J}}, \frac{\partial f_{\omega}}{\partial Z^{I}}\right\rangle \\
& +\frac{\gamma \sqrt{\mathbf{h}}}{q}(B u)^{J} \int \mu_{L}^{K}\left(\frac{\partial^{2} f_{\omega}}{\partial Z^{J} \partial Z^{K}} \frac{\partial f_{\omega}}{\partial Z^{I}}-\frac{\partial^{2} f_{\omega}}{\partial Z^{i} \partial Z^{J}} \frac{\partial f_{\omega}}{\partial Z^{K}}\right) d Z \\
& +2 \omega \gamma \sqrt{\mathbf{h}}\left\langle\mu^{0} f_{\omega}, \frac{\partial f_{\omega}}{\partial Z^{I}}\right\rangle \\
= & \sqrt{\mathbf{h}}\left(P B^{-1}+\gamma Q B^{-1}\right)_{I}^{a}\left(\partial_{T}\left(\frac{\left\|\nabla f_{\omega}\right\|^{2}}{n q} \gamma u_{a}\right)+\omega\left\|f_{\omega}\right\|^{2} \partial_{T}\left(\frac{\omega}{q} \gamma u_{a}\right)\right) .
\end{aligned}
$$

For $i \in\{1, \ldots, n\}$

$$
\begin{aligned}
\left\langle b_{n+i}, j_{2}\right\rangle+\left\langle a_{n+i}, j_{1}\right\rangle= & -\frac{\sqrt{\mathbf{h}}}{q}(\gamma P+Q)_{i}^{j} B_{j}^{J} \tilde{\mu}^{I}\left\langle\frac{\partial f_{\omega}}{\partial Z^{I}}, \frac{\partial f_{\omega}}{\partial Z^{J}}\right\rangle \\
& +2 \frac{\gamma \sqrt{\mathbf{h}}}{q}(B u)^{K} \tilde{\mu}^{I}\left\langle\frac{\partial^{2} f_{\omega}}{\partial Z^{I} \partial Z^{K}}, B_{a}^{J} \zeta_{i}^{a} \frac{\partial f_{\omega}}{\partial Z^{J}}\right\rangle \\
& +2 \omega^{2} \gamma^{2} \frac{\sqrt{\mathbf{h}}}{q} \tilde{\mu}^{I}\left\langle(\gamma P+Q)_{i c}\left(B^{-1} Z\right)^{c} f_{\omega}, \frac{\partial f_{\omega}}{\partial Z^{I}}\right\rangle \\
& +2 \omega \gamma \sqrt{\mathbf{h}} \tilde{\mu}^{0}\left\langle f_{\omega}, B_{a}^{J} \zeta_{j}^{a} \frac{\partial f_{\omega}}{\partial Z^{J}}\right\rangle \\
& -2 \omega \gamma^{2} \frac{\sqrt{\mathbf{h}}}{q^{2}}(B u)^{K} \tilde{\mu}^{0}\left\langle\frac{\partial f_{\omega}}{\partial Z^{K}},(\gamma P+Q)_{i c}\left(B^{-1} Z\right)^{c} f_{\omega}\right\rangle \\
= & -\gamma^{2} \frac{\sqrt{\mathbf{h}}}{q}\left(\frac{\left\|\nabla f_{\omega}\right\|^{2}}{n}+\omega^{2}\left\|f_{\omega}\right\|^{2}\right)\left(\gamma^{2} P+Q\right)_{i j} \partial_{T} \alpha^{j} .
\end{aligned}
$$

Terms involving $f_{1}, f_{2}$. To calculate the quantities $\left\langle a_{A}, f_{1}\right\rangle+\left\langle b_{A}, f_{2}\right\rangle$, first of all notice that for $A \in\{-1, n+1, \ldots, 2 n\} \Re b_{A}$ is an even function of $Z$ and $\Re a_{A}$ is odd, while the situation is reversed for the imaginary parts. From this observation it follows directly that for these values of $A$ it is possible to write

$$
\begin{aligned}
\left\langle a_{A}, f_{1}\right\rangle+\left\langle b_{A}, f_{2}\right\rangle= & \frac{1}{\varepsilon} \int\left(\left(\frac{p^{\varepsilon}}{\sqrt{\mathbf{g}^{\varepsilon}}}-\frac{q}{\sqrt{\mathbf{h}}}\right) F^{0}(Z)+\left(p^{\varepsilon} \sqrt{\mathbf{g}^{\varepsilon}}-q \sqrt{\mathbf{h}}\right) F^{1}(Z)\right. \\
& \left.+\left(p^{\varepsilon} \sqrt{\mathbf{g}^{\varepsilon}}\left(g^{\varepsilon}\right)^{i j}-q \sqrt{\mathbf{h}} h^{i j}\right) F_{i j}(Z)\right)
\end{aligned}
$$

where all the $F^{\prime} s$ are even functions of $Z$. In accordance with the remarks above about using $Z$ instead of $x$ as independent variable in these formulae

$$
p^{\varepsilon}=p\left(\varepsilon \xi+\varepsilon\left(\gamma^{-1} P+Q\right)\left(B^{-1} Z\right)\right) \quad q=p(\varepsilon \xi)
$$


etc.; therefore by Lemma A.10 these terms, which are continuous functions of $t, \lambda$ are $\mathrm{O}(\varepsilon)$ and lie in the class $\mathfrak{E}_{1}$ and satisfy a bound as in (2.20) with $c$ depending also on $K_{2}$. Thus these terms contribute to $\varepsilon \tilde{F}_{A}^{(0)}$ on the right hand side of the modulation equations.

For $A=0$ it follows by inspection that $\left\langle a_{A}, f_{1}\right\rangle+\left\langle b_{A}, f_{2}\right\rangle=0$, thus it is left to compute this expression in the case $A=1, \ldots, n$. The final answer is

$$
\begin{aligned}
\left\langle b_{I}, f_{2}\right\rangle+\left\langle a_{I}, f_{1}\right\rangle= & \frac{1}{2} \mathbf{m}_{\omega}\left(\gamma^{-1} P B^{-1}+Q B^{-1}\right)_{I}^{k} \\
& \times\left(h_{, k}^{i j} \frac{\gamma^{2}|u|_{h}^{2}}{q^{2}} q \sqrt{\mathbf{h}} P_{i j}+2 \gamma^{2} \sqrt{\mathbf{h}} q_{, k}\right)+\mathrm{O}(\varepsilon),
\end{aligned}
$$

where the $\mathrm{O}(\varepsilon)$ term is a continuous function of $t, \lambda$ which satisfies a bound as in (2.20) (in which $c$ depends also on $K_{2}$ ) and so contributes to $\varepsilon \tilde{F}_{A}^{(0)}$ in the modulation equations.

To prove this it is helpful to split up the various terms carefully. For $I \in\{1, \ldots, n\}$

$$
\begin{aligned}
\left\langle b_{I}, f_{2}\right\rangle= & -\frac{1}{\varepsilon} \int\left(p^{\varepsilon} \sqrt{\mathbf{g}^{\varepsilon}}\left(g^{\varepsilon}\right)^{i j}-q \sqrt{\mathbf{h}} h^{i j}\right)\left\langle\left(\nabla_{x}\right)_{i}\left(\frac{\partial f_{\omega}}{\partial Z^{I}} e^{i \Theta}\right),\left(\nabla_{x}\right)_{j}\left(f_{\omega} e^{i \Theta}\right)\right\rangle d Z \\
& -\frac{1}{\varepsilon} \int\left(p^{\varepsilon} \sqrt{\mathbf{g}^{\varepsilon}}-q \sqrt{\mathbf{h}}\right)\left\langle\frac{\partial f_{\omega}}{\partial Z^{I}},\left(m^{2}-\beta\left(f_{\omega}\right)\right) f_{\omega}\right\rangle d Z \\
= & E_{I 1}+E_{I 2}, \\
\left\langle a_{I}, f_{1}\right\rangle= & \frac{1}{\varepsilon} \int \frac{\sqrt{\mathbf{h}}}{q}\left(\frac{p^{\varepsilon}}{\sqrt{\mathbf{g}^{\varepsilon}}}-\frac{q}{\sqrt{\mathbf{h}}}\right)\left\langle\gamma(B u) \cdot \nabla_{Z} \frac{\partial f_{\omega}}{\partial Z^{I}}-i \omega q \gamma \frac{\partial f_{\omega}}{\partial Z^{I}}, i \omega \gamma \sqrt{\mathbf{h}} f_{\omega}(Z)\right. \\
& \left.-\frac{\gamma \sqrt{\mathbf{h}}}{q} \frac{\partial f_{\omega}}{\partial Z^{I}}(B u)^{I}\right\rangle d Z \\
= & E_{I 3} .
\end{aligned}
$$

Using the proof of Lemma A.10 and integration by parts it is possible to write (for $I \in\{1, \ldots, n\}$ and $\alpha=1,2,3)$

$$
E_{I \alpha}=\left(\gamma^{-1} P B^{-1}+Q B^{-1}\right)_{I}^{k} E_{k \alpha}^{0}+\varepsilon E_{k \alpha}^{1}
$$

where

$$
\begin{aligned}
& E_{k 1}^{0}=\left.\frac{1}{2}\left(\nabla_{x}\right)_{k}\left(p^{\varepsilon} \sqrt{\mathbf{g}^{\varepsilon}}\left(g^{\varepsilon}\right)^{i j}\right)\right|_{x=\xi}\left(\frac{\left\|\nabla f_{\omega}\right\|^{2}}{n}\left(\gamma^{2} P_{i j}+Q_{i j}\right)+\frac{\omega^{2} \gamma^{2}}{q^{2}} u_{i} u_{j}\left\|f_{\omega}\right\|^{2}\right), \\
& E_{k 2}^{0}=\left.\frac{1}{2}\left(\nabla_{x}\right)_{k}\left(p^{\varepsilon} \sqrt{\mathbf{g}^{\varepsilon}}\right)\right|_{x=\xi} \int\left(m^{2} f_{\omega}^{2}-G\left(f_{\omega}\right)\right) d Z, \\
& E_{k 3}^{0}=\left.\frac{1}{2}\left(\nabla_{x}\right)_{k}\left(\frac{p^{\varepsilon}}{\sqrt{\mathbf{g}^{\varepsilon}}}\right)\right|_{x=\xi}\left(\frac{\left\|\nabla f_{\omega}\right\|^{2}}{n} \frac{\mathbf{h} \gamma^{2}}{q^{2}}|u|_{h}^{2}+\omega^{2} \gamma^{2} \mathbf{h}\left\|f_{\omega}\right\|^{2}\right),
\end{aligned}
$$

with

$$
G^{\prime}(t)=2 t \beta(t)
$$

Notice that here, and in the following, after differentiation the terms involving $p^{\varepsilon}, g^{\varepsilon}$ are to be evaluated at $x=\xi$, using the notation (1.10). Lemma A.10 implies that the remainder terms $\varepsilon E_{k \alpha}^{1}$ are indeed $\mathrm{O}(\varepsilon)$ and satisfy a bound as in (2.20) (in which $c$ depends also on $K_{2}$ ). Since they are continuous functions of $t, \lambda$ they lie in the class $\mathfrak{E}_{1}$ and so contribute to $\tilde{F}_{A}^{(0)}$ in the inhomogeneous terms. 
To evaluate the dominant terms, use the Pohazaev identity (see e.g. [23])

$$
(2-n)\left\|\nabla f_{\omega}\right\|^{2}-n\left(m^{2}-\omega^{2}\right)\left\|f_{\omega}\right\|^{2}+n \int G\left(f_{\omega}\right)=0
$$

to combine them as

$$
E_{1 k}^{0}+E_{2 k}^{0}+E_{3 k}^{0}=\frac{1}{2}\left(\frac{\left\|\nabla f_{\omega}\right\|^{2}}{n} A+\omega^{2}\left\|f_{\omega}\right\|^{2} B\right)
$$

where (for fixed $k$ )

$$
\begin{aligned}
& A=\left(\nabla_{x}\right)_{k}\left(p^{\varepsilon} \sqrt{\mathbf{g}^{\varepsilon}}\left(g^{\varepsilon}\right)^{i j}\right)\left(\gamma^{2} P_{i j}+Q_{i j}\right)+(2-n)\left(\nabla_{x}\right)_{k}\left(p^{\varepsilon} \sqrt{\mathbf{g}^{\varepsilon}}\right)+\frac{\mathbf{h} \gamma^{2}}{q^{2}}|u|_{h}^{2}\left(\nabla_{x}\right)_{k}\left(\frac{p^{\varepsilon}}{\sqrt{\mathbf{g}^{\varepsilon}}}\right), \\
& B=\left(\nabla_{x}\right)_{k}\left(p^{\varepsilon} \sqrt{\mathbf{g}^{\varepsilon}}\left(g^{\varepsilon}\right)^{i j}\right) \frac{\gamma^{2}|u|_{h}^{2}}{q^{2}} P_{i j}+\left(\nabla_{x}\right)_{k}\left(p^{\varepsilon} \sqrt{\mathbf{g}^{\varepsilon}}\right)+\gamma^{2} \mathbf{h}\left(\nabla_{x}\right)_{k}\left(\frac{p^{\varepsilon}}{\sqrt{\mathbf{g}^{\varepsilon}}}\right) .
\end{aligned}
$$

Here it is understood that $p^{\varepsilon}, g^{\varepsilon}$ are evaluated at $x=\xi$ (after differentiation if need be). Calculation shows that these are in fact equal:

$$
A=B=\left(\nabla_{x}\right)_{k}\left(g^{\varepsilon}\right)^{i j}\left(\frac{\gamma^{2}|u|_{h}^{2}}{q^{2}} p^{\varepsilon} \sqrt{\mathbf{g}^{\varepsilon}} P_{i j}\right)+2 \gamma^{2} \sqrt{\mathbf{g}^{\varepsilon}}\left(\nabla_{x}\right)_{k} p^{\varepsilon}
$$

which finally gives (A.45) (by way of the definition in (1.59)).

\section{A.10. Local existence}

In this appendix basic estimates are given which imply the validity of the local existence hypotheses (WP-1) and (WP-2) of Section 1.7 will be given. It is convenient to rewrite (1.11) as

$$
\square_{\underline{g}} \phi=\mathcal{F}, \quad \phi(0)=\phi_{0}, \quad \phi_{t}(0)=\psi_{0},
$$

with $\mathcal{F}=\mathcal{F}(\phi)$.

THEOREM A.14. - Assume that $\mathcal{F}: \mathbb{C} \rightarrow \mathbb{C}$ satisfies:

(i) $|\mathcal{F}(\phi)| \leqslant M\left(|\phi|+|\phi|^{p}\right)$,

(ii) $|\mathcal{F}(\phi)-\mathcal{F}(\tilde{\phi})| \leqslant L\left(|\phi-\tilde{\phi}|+\left(|\phi|^{p-1}+|\tilde{\phi}|^{p-1}\right)|\phi-\tilde{\phi}|\right)$,

where $p \in(1, \infty)$ if $n=1,2$ and $p \in\left(\frac{n}{n-2}, \frac{n+2}{n-2}\right)$ if $n \geqslant 3$. Then the Cauchy problem for (A.49) is well-posed in $H^{1} \times L^{2}$ and satisfies the hypotheses (WP1)-(WP2) of Section 1.7.

The theorem is proved by showing convergence of the Picard iterates in the corresponding topology by means of the following two estimates for the inhomogeneous problem (A.49) (in which $\mathcal{F}$ is now regarded as a given function):

$$
\begin{aligned}
& \sup _{s \in[0, t]}\|(\phi, \psi)\|_{H^{1} \times L^{2}} \leqslant c_{1}\left(e^{c_{2} t}\left\|\left(\phi_{0}, \psi_{0}\right)\right\|_{H^{1} \times L^{2}}+\|\mathcal{F}\|_{L^{1}\left([0, t] ; L^{2}\right)}\right), \\
& \|\phi\|_{L^{Q}\left([0, t] ; L^{R}\right)} \leqslant c_{3}\left(\left\|\left(\phi_{0}, \psi_{0}\right)\right\|_{H^{1} \times L^{2}}+\|\mathcal{F}\|_{L^{1}\left([0, t] ; L^{2}\right)}\right) .
\end{aligned}
$$

Here the constants $c_{i}$ depend on the constant $K_{2}$ introduced in Section 1.2 and in (A.51) $R \in\left(\frac{2 n}{n-2}, \frac{2(n+1)}{n-3}\right], Q=\frac{2 R}{(n-2) R-2 n}, n>2$ and $t \leqslant t_{1}$ where $t_{1}$ is a positive number depending upon $K_{2}$. The first of these follows from the energy identity while the second is a variable coefficient Strichartz estimate proved in [25, Corollary 5] for the case of $C^{2}$ metrics relevant here. 


\section{A.11. Proper reference frames}

In this appendix a local co-ordinate system corresponding to an observer moving along the curve $\xi(t)$ will be used to clarify the meaning of the expressions for the dominant term in (1.12). The construction of the co-ordinate system is given in [14, Section 13.6]; see also [11]. First of all let $v=\frac{d \xi}{d t}$ be the $n$-velocity along the curve and introduce the proper time $\tau(t)$ by

$$
\tau(t)=\int_{0}^{t} \frac{q\left(t^{\prime}\right)}{\gamma\left(t^{\prime}\right)} d t^{\prime}
$$

where, as usual, $q(t)=p(t, \xi(t)), h_{i j}(t)=g_{i j}(t, \xi(t))$ and $\gamma(t)=\bar{\gamma}\left(1-|v|_{h} / q\right)$. The idea is to find a local system of co-ordinates $\left(\hat{x}^{0}=\hat{t}, \hat{x}\right)$ in a tube surrounding the curve $(t, \xi(t))$ in which $\tau(t), \hat{x}=0$ corresponds to $(t, x=\xi(t))$. As explained in [14] this can be done by using an orthonormal frame $E_{0}, \ldots, E_{n}$ to $T \mathbb{M}$ along the curve in which $E_{0}$ is the spacetime velocity vector of the curve $\left(\frac{\gamma}{q}, \frac{\gamma v}{q}\right)$ and $E_{1}, \ldots, E_{n}$ are (non-parallel) propagated in such a way as to preserve orthonormality (see [14, Eq. 13.60, Section 13.6]. The co-ordinate system is then produced from the geodesic flow. Let $\Phi((t, \xi(t)), \sigma, \mathbf{n})$ be the geodesic emanating from the point $\left(t_{0}, \xi\left(t_{0}\right)\right.$, parametrised by arc-length $\sigma$, in the direction $\mathbf{n}=n^{i} E_{i}$ : then if $(t, x)=\Phi\left(\left(t_{0}, \xi\left(t_{0}\right)\right), \sigma, \mathbf{n}\right)$, the new co-ordinates of this point are $\left(\hat{t}, \hat{x}^{i}\right)=\left(\tau\left(t_{0}\right), \sigma n^{i}\right)$. Observe that by construction the co-ordinates of a point $(t, \xi(t))$ on the curve are $(\hat{t}=\tau(t), \hat{x}=0)$. For $\underline{g}$ a $C^{3}$ metric the geodesic flow is $C^{2}$, and from this and the expression for $E_{0}$ it follows that for $|x-\xi(t)|$ sufficiently small the new time co-ordinate $\hat{t}(t, x)$ satisfies:

$$
\left|\hat{t}(t, x)-\tau(t)+\frac{1}{q(t)} \gamma(t)\langle v, x-\xi(t)\rangle_{h}\right| \leqslant C|x-\xi(t)|^{2} .
$$

From this it follows by consideration of the pseudo-Riemannian distance between $(t, \xi(t))$ and $(t, x)$ in both co-ordinate systems that

$$
\left.|| \hat{x}(t, x)\right|^{2}-\left|\gamma(t) P_{v, h}(x-\xi)+Q_{v}(x-\xi)\right|_{h}^{2}|\leqslant C| x-\left.\xi(t)\right|^{3} .
$$

Thus to conclude, the function $\phi_{0}$ defined in (1.12) pulls back under this change of co-ordinates to $e^{i \omega \hat{t}} f_{\omega}(|\hat{x}|)+o(1)$, in a neighbourhood of the curve $\xi(t)$, as stated in the remark following (1.12).

\section{REFERENCES}

[1] ANDERSON D.L.T., Stability of time-dependent particle-like solutions in nonlinear field theories, II, J. Math. Phys. 12 (1971) 945-952.

[2] BeReStYCKi H., LiONS P.-L., Nonlinear scalar field equations. I. Existence of a ground state, Arch. Rat. Mech. Anal. 82 (1983) 313-345.

[3] Berestycki H., Lions P.-L., Peletier L., An ODE approach to existence of positive solutions for semilinear problems in $R^{n}$, Indiana Univ. Math. J. 30 (1983) 141-157.

[4] Bronski J., Jerrard R., Soliton dynamics in a potential, Math. Res. Lett. 7 (2000) 329-342.

[5] Coffman C.V., Uniqueness of the ground state solution for $\Delta u-u+u^{3}=0$ and a variational characterization of other solutions, Arch. Rat. Mech. Anal. 46 (1972) 81-95.

[6] Folland G.B., Introduction to Partial Differential Equations, Princeton University Press, Princeton, NJ, 1995.

[7] Grillakis M., Shatah J., Strauss W., Stability theory of solitary waves in the presence of symmetry, I, J. Funct. Anal. 74 (1987) 160-197. 
[8] Grillakis M., Shatah J., Strauss W., Stability theory of solitary waves in the presence of symmetry, II, J. Funct. Anal. 94 (1990) 308-348.

[9] KeraAni S., Semiclassical limit of a class of Schrödinger equations with potential, Comm. Partial Differential Equations 27 (2002) 693-704.

[10] LeE T.D., Particle Physics and Introduction to Field Theory, Harwood, Chur, 1984.

[11] Manasse F., Misner C., Fermi normal coordinates and some basic concepts in differential geometry, J. Math. Physics 4 (1963) 735-745.

[12] Marsden J., Lectures on Mechanics, in: LMS Lecture Note Series, vol. 174, LMS, Cambridge, 1992.

[13] MCLEOD K., Uniqueness of positive radial solutions of $\Delta u+f(u)=0$ in $R^{n}$, Trans. Amer. Math. Soc. 339 (1993) 495-505.

[14] Misner C.W., Thorne K.S., Wheeler J.A., Gravitation, Freeman, San Francisco, 1973.

[15] Peletier L., Serrin J., Uniqueness of positive solutions of semilinear equations in $R^{n}$, Arch. Rat. Mech. Anal. 81 (2) (1983) 181-197.

[16] Shatah J., Stable standing waves of nonlinear Klein-Gordon equations, Comm. Math. Phys. 91 (1983) 313-327.

[17] Shatah J., Strauss W., Instability of nonlinear bound states, Comm. Math. Phys. 100 (1985) 173190.

[18] Strauss W., Existence of solitary waves in higher dimensions, Comm. Math. Phys. 55 (1977) 149162.

[19] Strauss W., Nonlinear Wave Equations, AMS, Providence, RI, 1989.

[20] StUART D., Solitons on pseudo-Riemannian manifolds I, Comm. PDE 1815-1838 (1998) 149-191.

[21] StuarT D.M.A., Geodesics and the Einstein nonlinear wave system, University of Cambridge preprint.

[22] StuART D.M.A., Solitons on pseudo-Riemannian manifolds: stability and motion, Electron. Res. Announc. Amer. Math. Soc. 6 (2000) 75-89.

[23] StUART D.M.A., Modulational approach to stability of non-topological solitons in semilinear wave equations, J. Math. Pures Appl. 80 (1) (2001) 51-83.

[24] Stuart D.M.A., Geodesics and the Einstein-nonlinear wave system, C. R. Acad. Sci. Paris Ser. I 336 (2003) 615-618.

[25] TATARU D., Strichartz estimates for second order hyperbolic operators with nonsmooth coefficients II, Amer. J. Math. 123 (3) (2001) 385-423.

[26] Weinberg S., Gravitation and Cosmology, Wiley, New York, 1972.

[27] Weinstein M.I., Nonlinear Schrödinger equations and sharp interpolation estimates, Comm. Math. Phys. 87 (4) (1983) 567-576.

[28] Weinstein M.I., Modulational stability of ground states of nonlinear Schrödinger equations, SIAM J. Math. Anal. 16 (1985) 472-491.

(Manuscrit reçu le 8 décembre 2002 ; accepté, après révision, le 10 juillet 2003.)

\footnotetext{
David M.A. STUART

University of Cambridge,

CMS Wilberforce Road,

Cambridge CB3 OWA, UK

E-mail: D.M.A.Stuart@damtp.cam.ac.uk
} 